\title{
Light-Cone Quantization: Foundations and Applications
}

\author{
Thomas Heinzl \\ Friedrich-Schiller-Universität Jena, Theoretisch-Physikalisches Institut, Max-Wien- \\ Platz 1, D-07743 Jena
}

\begin{abstract}
These lecture notes review the foundations and some applications of lightcone quantization. First I explain how to choose a time in special relativity. Inclusion of Poincaré invariance naturally leads to Dirac's forms of relativistic dynamics. Among these, the front form, being the basis for light-cone quantization, is my main focus. I explain a few of its peculiar features such as boost and Galilei invariance or separation of relative and center-of-mass motion. Combining light-cone dynamics and field quantization results in light-cone quantum field theory. As the latter represents a first-order system, quantization is somewhat nonstandard. I address this issue using Schwinger's quantum action principle, the method of Faddeev and Jackiw, and the functional Schrödinger picture. A finite-volume formulation, discretized light-cone quantization, is analysed in detail. I point out some problems with causality, which are absent in infinite volume. Finally, the triviality of the light-cone vacuum is established. Coming to applications, I introduce the notion of light-cone wave functions as the solutions of the light-cone Schrödinger equation. I discuss some examples, among them nonrelativistic Coulomb systems and model field theories in two dimensions. Vacuum properties (like chiral condensates) are reconstructed from the particle spectrum obtained by solving the light-cone Schrödinger equation. In a last application, I make contact with phenomenology by calculating the pion wave function within the Nambu and Jona-Lasinio model. I am thus able to predict a number of observables like the pion charge and core radius, the r.m.s. transverse momentum, the pion structure function and the pion distribution amplitude. The latter turns out to be the asymptotic one.
\end{abstract}

\section{Introduction}

The nature of elementary particles calls for a synthesis of relativity and quantum mechanics. The necessity of a quantum treatment is quite evident in view of the microscopic scales involved which are several orders of magnitude smaller than in atomic physics. These very scales, however, also require a relativistic formulation. A typical hadronic scale of $1 \mathrm{fm}$, for instance, corresponds to momenta of the order of $p \sim \hbar c / 1 \mathrm{fm} \simeq 200 \mathrm{MeV}$. For particles with masses $M \lesssim 1 \mathrm{GeV}$, this implies sizable velocities $v \simeq p / M \gtrsim 0.2 c$.

It turns out that the task of unifying the principles of quantum mechanics and relativity is not a straightforward one. One can neither simply extend ordinary quantum mechanics to include relativistic physics nor quantize relativistic mechanics using the ordinary correspondence rules. Nevertheless, Dirac and others have succeeded in formulating what is called "relativistic quantum 
mechanics", which has become a subject of text books since - see e.g. (Bjorken and Drell 1964; Yndurain 1996). It should, however, be pointed out that this formulation, which is based on the concept of single-particle wave-functions and equations, is not really consistent. It does not correctly account for relativistic causality (retardation effects etc.) and the existence of antiparticles. As a result, one has to struggle with issues like the Klein paradox ${ }^{1}$, the definition of position operators (Newton and Wigner 1949) and the like.

The well-known solution to these problems is provided by quantum field theory, with an inherently correct description of antiparticles that entails relativistic causality. In contrast to single-particle wave mechanics, quantum field theory is a (relativistic) many body formulation that necessarily involves (anti-)particle creation and annihilation. Physical particle states are typically a superposition of an infinite number of 'bare' states, as any particle has a finite probability to emit or absorb other particles at any moment of time. A pion, for example, would be represented in terms of the following Fock expansion,

$$
|\pi\rangle=\psi_{2}|q \bar{q}\rangle+\psi_{3}|q \bar{q} g\rangle+\psi_{4}|q \bar{q} q \bar{q}\rangle+\ldots,
$$

where the $\psi_{n}$ are the probability amplitudes to find $n$ particles (quarks $q$, antiquarks $\bar{q}$ or gluons $g$ ) in the pion. With the advent of QCD, however, a conceptual difficulty concerning this many-particle picture has appeared. At low energy or momentum transfer, hadrons, the bound states of QCD, are reasonably described in terms of two or three constituent quarks and thus as few-body systems. These 'effective' quarks $Q$ are dressed so that they gain an effective mass of the order of $300-400 \mathrm{MeV}$. They are used as the basic degrees of freedom in the 'constituent quark model'. This model yields a reasonable mass spectroscopy of hadrons (Lucha et al. 1991; Mukherjee et al. 1993), but its foundations are not very well established theoretically. First, a nonrelativistic treatment of light hadrons is not justified (see above). Second, the model violates many symmetries of QCD (in particular chiral symmetry). Third, it is rather unclear how a constituent picture can arise in a quantum field theory such as QCD.

In principle, in order to confirm the constituent quark model, one would have to solve the 'QCD Schrödinger equation' for hadron states |hadron $\rangle$ of mass $M_{\mathrm{h}}$,

$$
\left.\left.H_{\mathrm{QCD}} \mid \text { hadron }\right\rangle=M_{\mathrm{h}} \mid \text { hadron }\right\rangle,
$$

and check whether the eigenstates are reasonably well described in terms of the constituent valence states $|Q \bar{Q}\rangle$ or $|Q Q Q\rangle$. This is a very hard problem. A more moderate goal would be to 'relativize' the constituent quark model, ideally in such a way that it respects the symmetries of QCD. I will discuss this attempt in detail at the end of these lectures.

To arrive at this point, there is, of course, some way to go. Let me start with the following claim. A particularly useful approach for our purposes is based on a somewhat unorthodox choice of the 'time arrow' within special relativity:

\footnotetext{
${ }^{1}$ For a nice recent discussion, see (Holstein 1998).
} 
instead of the ordinary 'Galileian time' $t$, I choose 'light-cone time', $x^{+} \equiv t-z / c$. In the course of these lectures ${ }^{2}$, this claim will be substantiated step by step.

I will begin with some general remarks on relativistic dynamics (Section 2). As a paradigm example I discuss the free relativistic particle which is the prototype of a reparametrization invariant system. I show that the choice of the time parameter is not unique as it corresponds to a gauge fixing, the purpose of which is to get rid of the reparametrization redundancies. By considering the stability subgroups of the Poincare group, one finds that there are essentially three reasonable choices of 'time' for a relativistic system, corresponding to Dirac's 'instant', 'point' and 'front' form, respectively. The latter choice is the basis of light-cone dynamics, the main features of which will be discussed in the last part of Section 2.

Section 3 is devoted to light-cone field quantization. I show how the Poincaré generators are defined in this case and utilize Schwinger's quantum action principle to derive the canonical commutators. This is the first method of quantization to be discussed. The relation between equal-time commutators, the field equations and their solutions for different initial and/or boundary conditions is clarified.

It turns out that light-cone field theories, being of first order in the velocity, generally are constrained systems which require a special treatment. I rederive the canonical light-cone commutators using a second method of quantization (based on phase space reduction) due to Faddeev and Jackiw. I extend this discussion to light-cone quantization in finite volume and point out possible problems with causality in this approach. Going back to infinite volume, I introduce a third method of quantization, the functional Schrödinger picture, and combine it with the light-cone formalism. I close this section with a discussion of the presumably most spectacular feature of light-cone quantum field theory, the triviality of the vacuum.

As a prelude to the applications I introduce the notion of light-cone wave functions in Section 4. I show how light-cone wave functions can be obtained by solving the light-cone Schrödinger equation. As examples, I discuss nonrelativistic wave functions as they occur in hydrogen-like systems, some model field theory in $1+1$ dimensions and a simple Gaussian model.

In Section 5, I finally make contact with phenomenology. I calculate the lightcone wave function of the pion within the Nambu and Jona-Lasinio model. This model is known to provide a good description of spontaneous chiral symmetry breaking, as it is governed by the same symmetry group as low-energy QCD. With the pion wave function at hand I derive a number of observables like the pion charge and core radius, the electromagnetic form factor, the r.m.s. transverse momentum and the pion structure function. I conclude with a calculation of the pion distribution amplitude.

\footnotetext{
${ }^{2}$ Naturally, these lectures cannot cover all aspects of light-cone field theory. For a comprehensive recent review on the subject see (Brodsky, Pauli, and Pinsky 1998).
} 


\section{Relativistic Particle Dynamics}

The physically motivated desire to describe hadrons as bound states of a small, fixed number of constituents is our rationale to go back and reanalyze the relation between Hamiltonian quantum mechanics and relativistic quantum field theory.

Quite generally, bound states are obtained by solving the Schrödinger equation,

$$
i \hbar \frac{\partial}{\partial \tau}|\psi(\tau)\rangle=H|\psi(\tau)\rangle,
$$

for normalized, stationary states,

$$
|\psi(\tau)\rangle=e^{-i E \tau}|\psi(0)\rangle .
$$

This leads to the bound-state equation

$$
H|\psi(0)\rangle=E|\psi(0)\rangle,
$$

where $E$ is the bound state energy. We would like to make this Hamiltonian formalism consistent with the requirements of relativity. It is, however, obvious from the outset that this procedure will not be manifestly covariant as it singles out a time $\tau$ (and an energy $E$, respectively). Furthermore, it is not even clear what the time $\tau$ really is as it does not have an invariant meaning.

\subsection{The Free Relativistic Point Particle}

To see what is involved it is sufficient to consider the classical dynamics of a free relativistic particle. We want to find the associated canonical formulation as a basis for subsequent quantization. We will proceed by analogy with the treatment of classical free strings which is described in a number of textbooks (Green et al. 1987; Polyakov 1987). Accordingly, the relativistic point particle may be viewed as an infinitely short string.

Recall that the action for a relativistic particle is essentially given by the arc length of its trajectory

$$
S=-m s_{12} \equiv-m \int_{1}^{2} d s .
$$

This action ${ }^{3}$ is a Lorentz scalar as

$$
d s=\sqrt{g_{\mu \nu} x^{\mu} x^{\nu}}
$$

is the (infinitesimal) invariant distance. We can rewrite the action (6) as

$$
S=-m \int_{1}^{2} d s \sqrt{\dot{x}_{\mu} \dot{x}^{\mu}} \equiv \int_{1}^{2} d s L(s),
$$

\footnotetext{
${ }^{3}$ We work in natural units, $\hbar=c=1$.
} 
in order to introduce a Lagrangian $L(s)$ and the four velocity $\dot{x}^{\mu} \equiv d x^{\mu} / d s$. The latter obeys

$$
\dot{x}^{2} \equiv \dot{x}_{\mu} \dot{x}^{\mu}=1
$$

as the arc length provides a natural parametrization. Thus, $\dot{x}^{\mu}$ is a time-like vector, and we assume in addition that it points into the future, $\dot{x}^{0}>0$. In this way we guarantee relativistic causality ensuring that a real particle passing through a point $P$ will always propagate into the future light cone based at $P$.

We proceed with the canonical formalism by calculating the canonical momenta as

$$
p^{\mu}=-\frac{\partial L}{\partial \dot{x}_{\mu}}=m \dot{x}^{\mu}
$$

These are not independent, as can be seen by calculating the square using (9),

$$
p^{2}=m^{2} \dot{x}^{2}=m^{2},
$$

which, of course, is the usual mass-shell constraint. This constraint indicates that the Lagrangian $L(s)$ defined in (8) is singular, so that its Hessian $W^{\mu \nu}$ with respect to the velocities,

$$
W^{\mu \nu} \equiv \frac{\partial^{2} L}{\partial \dot{x}_{\mu} \partial \dot{x}_{\nu}}=-\frac{m}{\sqrt{\dot{x}^{2}}}\left(g^{\mu \nu}-\frac{\dot{x}^{\mu} \dot{x}^{\nu}}{\dot{x}^{2}}\right)=-m\left(g^{\mu \nu}-\dot{x}^{\mu} \dot{x}^{\nu}\right),
$$

is degenerate. It has a zero mode given by the velocity itself,

$$
W^{\mu \nu} \dot{x}_{\nu}=0 \text {. }
$$

The Lagrangian being singular implies that the velocities cannot be uniquely expressed in terms of the canonical momenta. This, however, is not obvious from (10), as we can easily solve for the velocities,

$$
\dot{x}^{\mu}=p^{\mu} / m \text {. }
$$

But if one now calculates the canonical Hamiltonian,

$$
H_{c}=-p_{\mu} \dot{x}^{\mu}-L=-m \dot{x}^{2}+m \dot{x}^{2}=0,
$$

one finds that it is vanishing! It therefore seems that we do not have a generator for the time evolution of our dynamical system. In the following, we will analyze the reasons for this peculiar finding.

First of all we note that the Lagrangian is homogeneous of first degree in the velocity,

$$
L\left(\alpha \dot{x}^{\mu}\right)=\alpha L\left(\dot{x}^{\mu}\right) .
$$

Thus, under a reparametrization of the world-line,

$$
s \mapsto s^{\prime}, \quad x^{\mu}(s) \mapsto x^{\mu}\left(s^{\prime}(s)\right),
$$


where the mapping $s \mapsto s^{\prime}$ is one-to-one with $d s^{\prime} / d s>0$ (orientation conserving), the Lagrangian changes according to

$$
L\left(d x^{\mu} / d s\right)=L\left(\left(d x^{\mu} / d s^{\prime}\right)\left(d s^{\prime} / d s\right)\right)=\left(d s^{\prime} / d s\right) L\left(d x^{\mu} / d s^{\prime}\right) .
$$

This is sufficient to guarantee that the action is invariant under (17), that is, reparametrization invariant,

$$
S=\int_{s_{1}}^{s_{2}} d s L\left(d x^{\mu} / d s\right)=\int_{s_{1}^{\prime}}^{s_{2}^{\prime}} d s^{\prime} \frac{d s}{d s^{\prime}} \frac{d s^{\prime}}{d s} L\left(d x^{\mu} / d s^{\prime}\right) \equiv S^{\prime},
$$

if the endpoints remain unchanged, $s_{1,2}=s_{1,2}^{\prime}$. On the other hand, $L$ is homogeneous of the first degree if and only if Euler's formula holds, namely

$$
L=\frac{\partial L}{\partial \dot{x}^{\mu}} \dot{x}^{\mu}=-p_{\mu} \dot{x}^{\mu} .
$$

This is exactly the statement (15), the vanishing of the Hamiltonian. Furthermore, if we differentiate (20) with respect to $\dot{x}^{\mu}$, we recover (13) expressing the singular nature of the Lagrangian. Summarizing, we have found the general result (Hanson et al. 1976; Sundermeyer 1982) that if a Lagrangian is homogeneous of degree one in the velocities, the action is reparametrization invariant, and the Hamiltonian vanishes. In this case, the momenta are homogeneous of degree zero, which renders the Lagrangian singular.

The reparametrization invariance is generated by the first class constraint (Dirac 1964; Sundermeyer 1982),

$$
\theta \equiv p^{2}-m^{2}=0
$$

as can be seen as follows. From the canonical one form $-g_{\mu \nu} p^{\mu} d x^{\nu}$ we read off the Poisson bracket

$$
\left\{x^{\mu}, p^{\nu}\right\}=-g^{\mu \nu},
$$

and calculate the change of the coordinate $x^{\mu}$,

$$
\begin{aligned}
\delta x^{\mu} & =\left\{x^{\mu}, \theta \delta \epsilon\right\}=-2 p^{\mu} \delta \epsilon=-2 m \dot{x}^{\mu} \delta \epsilon \equiv \dot{x}^{\mu} \delta \tau \\
& =x^{\mu}(\tau+\delta \tau)-x^{\mu}(\tau)=x^{\mu}\left(\tau^{\prime}\right)-x^{\mu}(\tau) .
\end{aligned}
$$

Thus, the reparametrization (17) is indeed generated by the constraint (21).

Reparametrization invariance can be viewed as a gauge or redundancy symmetry. The redundancy consists in the fact that a single trajectory (world-line) can be described by an infinite number of different parametrizations. The physical objects, the trajectories, are therefore equivalence classes obtained by identifying ('dividing out') all reparametrizations. The method to do so is well known, namely gauge fixing. For the case at hand, this corresponds to a particular choice of parametrization, or, more physically, to the choice of a time parameter $\tau$. This amounts to choosing a foliation of space-time into space and time. Minkowski space is thus decomposed into hypersurfaces of equal time, $\tau=$ const, 
which in general are three-dimensional objects, and the time direction 'orthogonal' to them. The time development thus continuously evolves the hypersurface $\Sigma_{0}: \tau=\tau_{0}$ into $\Sigma_{1}: \tau=\tau_{1}>\tau_{0}$. Put differently, initial conditions provided on $\Sigma_{0}$ together with the dynamical equations (being differential equations in $\tau$ ) determine the state of the dynamical system on $\Sigma_{1}$.

Practically, the $(3+1)$-foliation is done as follows. We introduce some arbitrary coordinates, $\xi^{\alpha}=\xi^{\alpha}(x)$, which may be curvilinear. We imagine that three of these, say $\xi^{i}, i=1,2,3$, parametrize the three-dimensional hypersurface $\Sigma$, so that the remaining one, $\xi^{0}$, represents the time variable, i.e. $\tau=\xi^{0}(x)$. This equation can equivalently be viewed as a gauge fixing condition.

The first question to be addressed is: what is a 'good' choice of time? There are two criteria to be met, namely existence and uniqueness. Existence means that the equal-time hypersurface $\Sigma$ should intersect any possible world-line, while uniqueness requires that it does so once and only once. Mathematically, uniqueness can be analysed in terms of the Faddeev-Popov (FP) 'operator', which is given by the Poisson bracket of the gauge fixing condition with the constraint (evaluated on $\Sigma$ ),

$$
\mathrm{FP} \equiv\left\{\xi^{0}(x), \theta\right\}=\left\{\xi^{0}(x), p^{2}\right\}=-2 \frac{\partial \xi^{0}}{\partial x^{\mu}} p^{\mu} \equiv-2 N \cdot p .
$$

Here, we have introduced the normal $N$ on $\Sigma$,

$$
N^{\mu}(x)=\left.\frac{\partial \xi^{0}(x)}{\partial x^{\mu}}\right|_{\Sigma}
$$

which will be important later on. The statement now is that uniqueness is achieved (for a single degree of freedom) if the FP operator does not vanish, i.e. if $N \cdot p \neq 0$. Generically, this means that the particle trajectory must not be parallel to the hypersurface $\Sigma$ of equal time.

As an aside we remark that this is completely analogous to the reasoning in standard gauge (field) theory. There, the constraint $\theta$ is given by Gauss's law which generates gauge transformations $A \rightarrow A+D \omega, D$ denoting the covariant derivative. For a gauge fixing $\chi[A]=0$, the equation corresponding to $(24)$ becomes

$$
\mathrm{FP}=\{\chi, \theta\}=\frac{\delta \chi}{\delta \omega}=\frac{\delta \chi}{\delta A} \frac{\delta A}{\delta \omega}=N \cdot D,
$$

where all (functional) derivatives are to be evaluated on the gauge fixing hypersurface, $\chi=0$.

Let us now perform an analysis of the canonical formalism for a general choice of hypersurface $\Sigma$. For this we need some notation. We write the line element as

$$
d s^{2}=g_{\mu \nu} d x^{\mu} d x^{\nu}=g_{\mu \nu} \frac{\partial x^{\mu}}{\partial \xi^{\alpha}} \frac{\partial x^{\nu}}{\partial \xi^{\beta}} d \xi^{\alpha} d \xi^{\beta} \equiv h_{\alpha \beta}(\xi) d \xi^{\alpha} d \xi^{\beta} .
$$

Introducing a vierbein $e_{\alpha}^{\mu}(\xi)$, the metric $h_{\alpha \beta}(\xi)$ is alternatively given by

$$
h_{\alpha \beta}(\xi)=g_{\mu \nu} e_{\alpha}^{\mu}(\xi) e_{\beta}^{\nu}(\xi) .
$$


The transformation $x \rightarrow \xi$ is well known from general relativity, where it corresponds to the transformation from a local inertial frame described by the flat metric $g_{\mu \nu}$ to a noninertial frame with coordinate dependent metric $h_{\alpha \beta}(\xi)$. For our purposes we write this metric in a $(3+1)$-notation as follows,

$$
h_{\alpha \beta}=\left(\begin{array}{cc}
h_{00} & h_{0 i} \\
h_{i 0} & h_{i j}
\end{array}\right) \equiv\left(\begin{array}{cc}
h_{00} & \boldsymbol{h}^{T} \\
\boldsymbol{h} & -H
\end{array}\right) .
$$

Of particular interest is the component $h_{00}$, which explicitly reads

$$
h_{00}=g_{\mu \nu} \frac{\partial x^{\mu}}{\partial \xi^{0}} \frac{\partial x^{\nu}}{\partial \xi^{0}}=g_{\mu \nu} e_{0}^{\mu} e^{\nu}{ }_{0} \equiv n^{2},
$$

where we have defined the unit vector in $\xi^{0}$-direction

$$
n^{\mu}=\frac{\partial x^{\mu}}{\partial \xi^{0}}=e_{0}^{\mu} \equiv \dot{x}^{\mu},
$$

which thus is the new four-velocity. It is related to the normal vector $N^{\mu}$ via

$$
n \cdot N=e^{\mu}{ }_{0} e_{\mu}^{0}=\frac{\partial \xi^{0}}{\partial x^{\mu}} \frac{\partial x^{\mu}}{\partial \xi^{0}}=1 .
$$

The normal vector $N$ enters the inverse metric which we write as follows,

$$
h^{\alpha \beta}=\left(\begin{array}{cc}
g_{00} & g_{0 i} \\
g_{i 0} & g_{i j}
\end{array}\right)=\left(\begin{array}{cc}
N^{2} & \boldsymbol{g}^{T} \\
\boldsymbol{g} & -G
\end{array}\right) .
$$

The $h_{i j}$ are the metric components associated with the hypersurface. The invariant distance element (27) thus becomes (with $h_{0 i} \equiv h_{i}$ ),

$$
\begin{aligned}
d s^{2} & =h_{00} d \xi^{0} d \xi^{0}+2 h_{0 i} d \xi^{0} d \xi^{i}+h_{i j} d \xi^{i} d \xi^{j}, \\
& =\left(n^{2}+2 h_{i} \frac{d \xi^{i}}{d \tau}+h_{i j} \frac{d \xi^{i}}{d \tau} \frac{d \xi^{j}}{d \tau}\right) d \tau^{2} \equiv h(\tau) d \tau^{2},
\end{aligned}
$$

where, in the second step, we have used that $\xi^{0}=\tau$. In the last identity we have defined a world-line metric or einbein

$$
h(\tau) \equiv \dot{x}^{2}=h_{\alpha \beta} \dot{\xi}^{\alpha} \dot{\xi}^{\beta},
$$

which expresses the arbitrariness in choosing a time by providing an (arbitrary) 'scale' for the velocity. Introducing the velocities expressed in the new coordinates, $w^{i} \equiv d \xi^{i} / d \tau$, the world-line metric can be written as

$$
h(\tau)=n^{2}+2 h_{i} w^{i}+h_{i j} w^{i} w^{j} .
$$

Let us develop a canonical formalism for a general choice of the einbein $h(\tau)$ corresponding to the gauge fixing $\tau=\xi^{0}(x)=0$. The Lagrangian becomes

$$
L(\tau)=-m \sqrt{h(\tau)},
$$


leading to the canonical momenta

$$
\pi_{\alpha}=-\frac{\partial L}{\partial \dot{\xi}^{\alpha}}=\frac{m}{\sqrt{h}} h_{\alpha \beta} \dot{\xi}^{\alpha}=e_{\alpha}^{\mu} p_{\mu} .
$$

We see that the einbein $h$ is appearing all over the place. The canonical Hamiltonian is expressed in terms of the inverse metric $h^{\alpha \beta}$,

$$
H_{c}=-\pi_{\alpha} \dot{\xi}^{\alpha}-L=-\frac{\sqrt{h}}{m}\left(h^{\alpha \beta} \pi_{\alpha} \pi_{\beta}-m^{2}\right)=0 .
$$

It vanishes (as it should) as it is proportional to the constraint,

$$
\theta=h^{\alpha \beta} \pi_{\alpha} \pi_{\beta}-m^{2}=p^{2}-m^{2}=0 .
$$

The FP operator also depends on the entries of the inverse metric (33), in particular the normal vector $N$,

$$
\mathrm{FP}=N^{2} \pi_{0}+g^{i} \pi_{i}
$$

After gauge fixing, the generator of $\tau$-evolution, $H_{\tau} \equiv \pi_{0}$, is obtained by solving the constraint (40) for $\pi_{0}$ which assumes the explicit form,

$$
N^{2} \pi_{0}^{2}+2 g^{i} \pi_{i} \pi_{0}-G^{i j} \pi_{i} \pi_{j}-m^{2}=0
$$

Depending on the value of $N^{2}$, we thus have to consider two different cases. The generic one is that the normal $N$ on $\Sigma$ is time-like, $N^{2}>0$. In this case, the mass-shell constraint is of second order in $\pi_{0}$, so that there are two distinct solutions,

$$
\pi_{0}=\frac{1}{N^{2}}\left\{-(\boldsymbol{g}, \boldsymbol{\pi}) \pm \sqrt{(\boldsymbol{g}, \boldsymbol{\pi})^{2}+N^{2}\left[(\boldsymbol{\pi}, G \boldsymbol{\pi})+m^{2}\right]}\right\} .
$$

Not unexpectedly, the 'problem' of two different signs in front of the square root arises (Gitman and Tyutin 1990). Within quantum mechanics, this is somewhat difficult to interpret. Upon 'second quantization', i.e. in the context of quantum field theory, one has, of course, the natural explanation in terms of antiparticles. As we will not quantize the relativistic point particle, the sign 'problem' is of no concern to us. A possible arbitrariness will be removed ad hoc by demanding $\pi_{0}>0$. With this additional condition the FP operator becomes

$$
\mathrm{FP}=-2 \sqrt{(\boldsymbol{g}, \boldsymbol{\pi})^{2}+N^{2}\left[(\boldsymbol{\pi}, G \boldsymbol{\pi})+m^{2}\right]},
$$

which is clearly nonvanishing for a massive particle, $m \neq 0$. A gauge fixing with $N^{2}>0$ is thus unique and leads to a well-defined description of the $\tau$-evolution.

The second case to be considered is in a sense degenerate. It corresponds to a light-like normal, $N^{2}=0$. In this case, the constraint (42) is only of first order in $\pi_{0}$ leading to a single solution,

$$
\pi_{0}=\frac{(\boldsymbol{\pi}, G \boldsymbol{\pi})+m^{2}}{(\boldsymbol{g}, \boldsymbol{\pi})} .
$$


As a result, there is no 'sign problem' and no 'ugly' square root. Conservation of difficulties, however, is at work, because it is no longer obvious whether the FP operator,

$$
\mathrm{FP}=-2(\boldsymbol{g}, \boldsymbol{\pi}),
$$

is different from zero. Clearly, this is absolutely necessary for (45) to represent a well-defined solution.

At this point, it should be mentioned that the results (43) and (45) are not yet the full story. The entries of the inverse metric, $N^{2}, \boldsymbol{g}$ and $G$ should actually be expressed in terms of the quantities $n^{2}, \boldsymbol{h}$ and $H$ defining the induced metric on $\Sigma$. So far, it is also not completely clear which choices of these parameters actually make sense physically. Of course, the normal $N$ should not be spacelike as this would imply that $\Sigma$ contains time-like directions and thus possible particle trajectories. In the next subsection I will give some criteria for reasonable choices of time.

Before we come to that let us apply the general formalism to the standard choice of 'Galileian' time, $\tau=\xi^{0}(x)=x^{0}=t$. In this case, the surface $\Sigma: t=0$ is an entirely space-like hyperplane with constant normal vector $N=(1, \mathbf{0})=n$. The other metric entries are $\boldsymbol{h}=\boldsymbol{g}=0$ and $H=G=\mathbb{1}$. The world-line metric (35) thus becomes

$$
h(t)=\dot{x}^{2}=1-v^{2} \equiv 1 / \gamma^{2},
$$

where $\gamma$ is the usual Lorentz contraction factor. The Hamiltonian is obtained in line with the second-order case above,

$$
H_{t}=N \cdot p=p^{0}=\sqrt{\mathbf{p}^{2}+m^{2}} \sim \mathrm{FP} .
$$

It generates the dynamics via the basic Poisson bracket $\left\{x^{i}, p^{j}\right\}=\delta^{i j}$ leading to

$$
\dot{x}^{i}=\left\{x^{i}, H_{t}\right\}=p^{i} / p^{0},
$$

with $p^{0}$ given by (48). Note that a well-defined time evolution requires a nonvanishing FP operator (which is proportional to $p^{0}$ ).

As already announced, we will discuss alternatives to this standard choice of time in the next subsection.

\subsection{Dirac's Forms of Relativistic Dynamics}

To address this issue it is not sufficient to consider only the $\tau$-development and the associated generator of time translations (i.e. the Hamiltonian) $H_{\tau}$. Instead, one has to refer to the full Poincaré group to be able to guarantee full relativistic invariance. The generators of the Poincaré group are the four momenta $P^{\mu}$ and the six operators $M^{\mu \nu}$ which combine angular momenta and boosts according to

$$
\begin{aligned}
L^{i} & =\frac{1}{2} \epsilon^{i j k} M^{j k}, \\
K^{i} & =M^{0 i},
\end{aligned}
$$


with $i, j, k=1,2,3$. These generators are elements of the Poincaré algebra which is defined by the Poisson bracket relations,

$$
\begin{aligned}
\left\{P^{\mu}, P^{\nu}\right\} & =0, \\
\left\{M^{\mu \nu}, P^{\rho}\right\} & =g^{\nu \rho} P^{\mu}-g^{\mu \rho} P^{\nu}, \\
\left\{M^{\mu \nu}, M^{\rho \sigma}\right\} & =g^{\mu \sigma} M^{\nu \rho}-g^{\mu \rho} M^{\nu \sigma}-g^{\nu \sigma} M^{\mu \rho}+g^{\nu \rho} M^{\mu \sigma} .
\end{aligned}
$$

It is well known that the momenta $P^{\mu}$ generate space-time translations and the $M^{\mu \nu}$ rotations and Lorentz boosts, cf. $(50,51)$. In the following we will only consider proper and orthochronous Lorentz transformations, i.e. we exclude space and time reflections.

Any Poincaré invariant dynamical theory describing e.g. the interaction of particles should provide a particular realization of the Poincaré algebra. For this purpose, the Poincaré generators are constructed out of the fundamental dynamical variables like positions, momenta, spins etc. An elementary realization of (52) is given as follows. Choose the space-time point $x^{\mu}$ and its conjugate momentum $p^{\mu}$ as canonical variables, i.e. adopt (22),

$$
\left\{x^{\mu}, p^{\nu}\right\}=-g^{\mu \nu} .
$$

The Poincaré generators are then found to be

$$
P^{\mu}=p^{\mu}, \quad M^{\mu \nu}=x^{\mu} p^{\nu}-x^{\nu} p^{\mu},
$$

as is easily confirmed by checking (52) using (53). An infinitesimal Poincaré transformation is thus generated by

$$
\delta G=-\frac{1}{2} \delta \omega_{\mu \nu} M^{\mu \nu}+\delta a_{\mu} P^{\mu}
$$

in the following way,

$$
\delta x^{\mu}=\left\{x^{\mu}, \delta G\right\}=\delta \omega^{\mu \nu} x_{\nu}+\delta a^{\mu}, \quad \delta \omega^{\mu \nu}=-\delta \omega^{\nu \mu} .
$$

The action of the Poincaré group on some scalar function $F(x)$ is thus given by

$$
\delta F=\{F, \delta G\}=\partial^{\mu} F \delta a_{\mu}-\frac{1}{2}\left(x^{\mu} \partial^{\nu}-x^{\nu} \partial^{\mu}\right) F \delta \omega_{\mu \nu} .
$$

Though the realization (54) is covariant, it has several shortcomings. It does not describe any interaction; for several particles the generators are simply the sum of the single particle generators. This point, however, is of minor importance to us, and will only be touched upon at the end of this subsection. The solution of the problem, as already mentioned in the introduction, is the framework of local quantum field theory. More importantly, the representation (54) does not take into account the mass-shell constraint, $p^{2}=m^{2}$, which we already know to guarantee relativistic causality as it generates the dynamics upon solving for $H_{\tau}$.

To remedy the situation we proceed as before by choosing a time variable $\tau$, i.e. a foliation of space-time into essentially space-like hypersurfaces $\Sigma$ with 
time-like or light-like normals $N$. We have seen that $\Sigma$ should be chosen in such a way that it intersects all possible world-lines once and only once (existence and uniqueness). Apart from this necessary consistency with causality the foliation appears quite arbitrary. However, given a particular foliation one can ask the question which of the Poincaré generators will leave the hypersurface $\Sigma$ invariant. The set of all such generators defines a subgroup of the Poincaré group called the stability group $G_{\Sigma}$ of $\Sigma$. The associated generators are called kinematical, the others dynamical. The latter map $\Sigma$ onto another hypersurface $\Sigma^{\prime}$ and thus involve the development in $\tau$. One thus expects that the dynamical generators will depend on the Hamiltonian (and, therefore, the interaction) which, by definition, is a dynamical quantity.

It is clear, however, that the stability group corresponding to a particular foliation will be empty if the associated hypersurface looks very irregular and thus does not have a high degree of symmetry. One therefore demands in addition that the stability group acts transitively on $\Sigma$ : any two points on $\Sigma$ can be connected by a transformation from $G_{\Sigma}$. With this additional requirement there are exactly five inequivalent classes of hypersurfaces (Leutwyler and Stern 1978) which are listed in Table 1.

Table 1. All possible choices of hypersurfaces $\Sigma: \tau=$ const with transitive action of the stability group $G_{\Sigma}$. d denotes the dimension of $G_{\Sigma}$, that is, the number of kinematical Poincaré generators; $\mathbf{x}_{\perp} \equiv\left(x^{1}, x^{2}\right)$.

\begin{tabular}{lllr}
\hline \hline name & $\Sigma$ & $\tau$ & $d$ \\
\hline instant & $x^{0}=0$ & $t$ & 6 \\
light front & $x^{0}+x^{3}=0$ & $t+x^{3} / c$ & 7 \\
hyperboloid & $x_{0}^{2}-\mathbf{x}^{2}=a^{2}>0, x^{0}>0$ & $\left(t^{2}-\mathbf{x}^{2} / c^{2}-a^{2} / c^{2}\right)^{1 / 2}$ & 6 \\
hyperboloid & $x_{0}^{2}-\mathbf{x}_{\perp}^{2}=a^{2}>0, x^{0}>0$ & $\left(t^{2}-\mathbf{x}_{\perp}^{2} / c^{2}-a^{2} / c^{2}\right)^{1 / 2}$ & 4 \\
hyperboloid & $x_{0}^{2}-x_{1}^{2}=a^{2}>0, x^{0}>0$ & $\left(t^{2}-x_{1}^{2} / c^{2}-a^{2} / c^{2}\right)^{1 / 2}$ & 4 \\
\hline \hline
\end{tabular}

The first three choices have already been found by (Dirac 1949) in his seminal paper on 'forms of relativistic dynamics'. He called the associated forms the 'instant', 'front' and 'point' forms, respectively. These are the most important choices as the remaining two forms have a rather small stability group and thus are not very useful. We have only listed them for the sake of completeness.

It is important to note that for all forms one has $\lim _{c \rightarrow \infty} \tau=t$, which means that in the nonrelativistic case there is only one possible foliation leading to the absolute Galileian time $t$. This is consistent with the fact that there is no limiting velocity in this case implying that particle trajectories can have arbitrary slope 
(tangent vector). Therefore, the hypersurface $\Sigma_{n r}: t=$ const is the only one intersecting all possible world-lines. For other choices, the criterion of existence introduced in the last subsection would be violated.

To decide which of the Poincaré generators are kinematical, we use the general formula (57) describing their action. Imagine that $\Sigma$ is given in the form $\Sigma$ : $\tau=\xi^{0}(x) \equiv F(x)$ as in Table 1. If $P^{\mu}$ or $M^{\mu \nu}$ are kinematical for some $\mu$ or $\nu$, then, for these particular superscripts, the components of the gradient and rotor of $F$ have to vanish on $\Sigma$,

$$
\partial^{\mu} F=0, \quad\left(x^{\mu} \partial^{\nu}-x^{\nu} \partial^{\mu}\right) F=0 .
$$

In terms of the normal vector $N$ these equations become

$$
N^{\mu}=0, \quad x^{\mu} N^{\nu}-x^{\nu} N^{\mu}=0,
$$

which again will hold for some of the superscripts $\mu$ and/or $\nu$, if $\Sigma$ has nontrivial stabilizer. The distinction between kinematical and dynamical is thus completely encoded in the normal vector $N$.

The choice of Galileian time $\tau=t$ is of course the most common one also in the relativistic case, and we have discussed it briefly at the end of the last subsection. To complete this discussion, we construct the associated representation of the Poincaré generators on $\Sigma: t=0$. The idea is again to explicitly saturate the constraint $p^{2}=m^{2}$ by solving for $H_{t}=p^{0}=N \cdot p=\left(\mathbf{p}^{2}+m^{2}\right)^{1 / 2}$, and setting $x^{0}=0$ in (54).

As a result, we obtain the following $(3+1)$-representation of the Poincaré generators,

$$
\begin{aligned}
& P^{i}=p^{i}, M^{i j}=x^{i} p^{j}-x^{j} p^{i}, \\
& P^{0}=H_{t}, M^{i 0}=x^{i} H_{t} .
\end{aligned}
$$

This outcome is as expected: Compared to $(54), p^{0}$ has been replaced by $H_{t}$, and $x^{0}$ has been set to zero. It should, however, be pointed out that for non-Cartesian coordinates the construction of the Poincaré generators is less straightforward.

Let us address the question of kinematical versus dynamical generators. In agreement with (58) and (59), one has

$$
N^{i}=0=x^{i} N^{j}-x^{j} N^{i}, \quad i, j=1,2,3,
$$

so that $\Sigma$ is both translationally and rotationally invariant confirming that the dimension of its stability group is six (cf. Table 1). On the other hand,

$$
\begin{array}{r}
N^{0}=1 \neq 0, \\
x^{0} N^{i}-x^{i} N^{0}=-x^{i} \neq 0,
\end{array}
$$

from which we read off that, apart from the Hamiltonian, also the boosts are dynamical, i.e., $\Sigma$ is not boost invariant. The latter fact is, of course, well known because the boosts mix space and time. Under a boost along the $\mathbf{n}$-direction with velocity $\mathbf{v}, t$ transforms as

$$
t \rightarrow t^{\prime}=t \cosh \omega+(\mathbf{n} \cdot \mathbf{x}) \sinh \omega
$$


where $\mathbf{n}=\mathbf{v} / v$ and $\omega$ is the rapidity, defined through $\tanh \omega=v$. From (64) it is evident that the hypersurface $\Sigma: t=0$ is not boost invariant.

In obtaining the representation (60), we make the Poincaré algebra compatible with the instant-form gauge-fixing constraint, $x^{0}=0$. An elementary calculation, using $\left\{x^{i}, p^{j}\right\}=\delta^{i j}$, indeed shows that the generators (60) really obey the bracket relations (52). We have already seen in (49) that the Hamiltonian $P^{0}=H_{t}$ generates the correct dynamics.

At this point it is getting time to really consider an alternative to the instant form in some detail.

\subsection{The Front Form}

For an arbitrary four-vector $a$ we perform the following transformation to lightcone coordinates,

$$
\left(a^{0}, a^{1}, a^{2}, a^{3}\right) \mapsto\left(a^{+}, a^{1}, a^{2}, a^{-}\right),
$$

where we have defined

$$
a^{+}=a^{0}+a^{3}, \quad a^{-}=a^{0}-a^{3} .
$$

We also introduce the transverse vector part of $a$ as

$$
\mathbf{a}_{\perp}=\left(a^{1}, a^{2}\right) .
$$

The metric tensor (29) becomes

$$
h_{\alpha \beta}=\left(\begin{array}{cc}
n^{2} & \boldsymbol{h}^{T} \\
\boldsymbol{h} & -H
\end{array}\right)=\left(\begin{array}{rrrr}
0 & 0 & 0 & 1 / 2 \\
0 & -1 & 0 & 0 \\
0 & 0 & -1 & 0 \\
1 / 2 & 0 & 0 & 0
\end{array}\right)
$$

The entries $1 / 2$ imply nonvanishing $\boldsymbol{h}$ and thus a slightly unusual scalar product,

$$
a \cdot b=g_{\mu \nu} a^{\mu} b^{\nu}=\frac{1}{2} a^{+} b^{-}+\frac{1}{2} a^{-} b^{+}-a^{i} b^{i}, \quad i=1,2 .
$$

According to Table 1, the front form is defined by choosing the hypersurface $\Sigma: x^{+}=0$, which is a plane tangent to the light-cone. It can equivalently be viewed as the wave front of a plane light wave traveling towards the positive $z$-direction. Therefore, $\Sigma$ is also called a light-front. The normal vector is

$$
N=(1,0,0,-1), \quad N^{2}=0,
$$

where $N$ has been written in ordinary coordinates. We see that $N^{+}=N^{0}+$ $N^{3}=0$ which implies that the normal $N$ to the hypersurface lies within the hypersurface (Neville and Rohrlich 1971; Rohrlich 1971). As $N$ is a light-like or null vector, $\Sigma$ is often referred to as a null-plane (Neville and Rohrlich 1971; Coester 1992). We have depicted the front-form hypersurface $\Sigma$ together with the light-cone in Fig. 1. 
Fig. 1. The hypersurface $\Sigma: x^{+}=0$ defining the front form. It is a null-plane tangential to the light-cone, $x^{2}=0$.

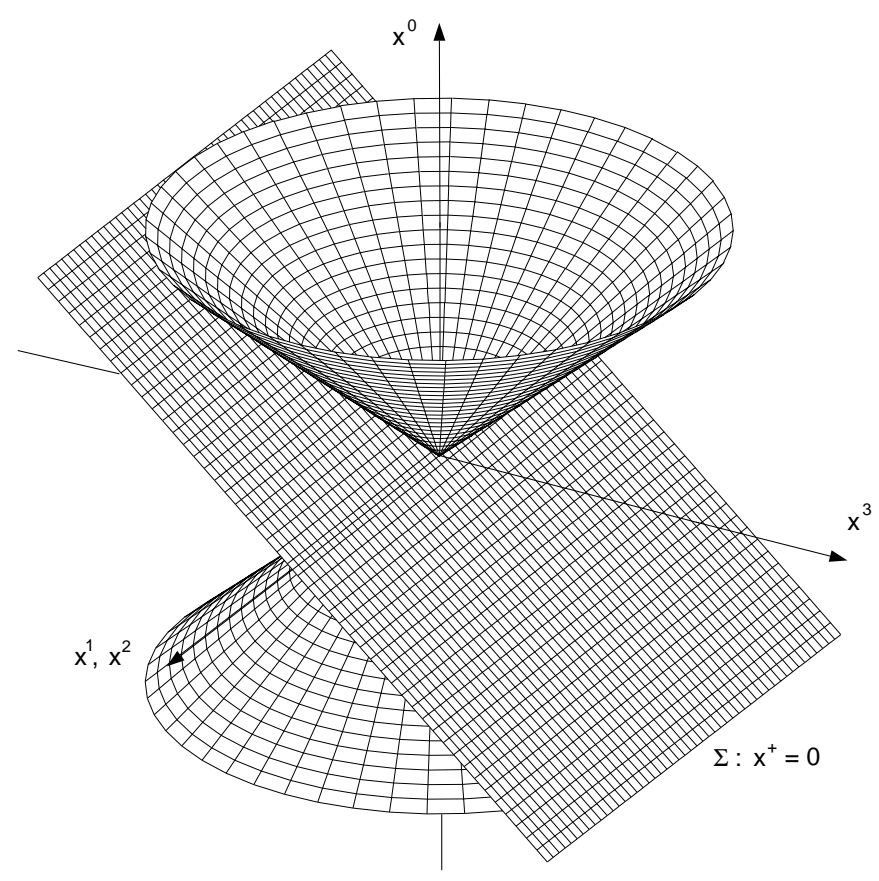

As is already obvious from (68), the unit vector in $x^{+}$-direction is another null-vector,

$$
n^{\mu}=\frac{\partial x^{\mu}}{\partial x^{+}}=\frac{1}{2}(1,0,0,1),
$$

so that $n \cdot N=1$ as it should. Given the scalar product (69), we infer the invariant distance element

$$
d s^{2}=g_{\mu \nu} d x^{\mu} d x^{\nu}=d x^{+} d x^{-}-d x^{i} d x^{i}=\left(\frac{d x^{-}}{d x^{+}}-\frac{d x^{i}}{d x^{+}} \frac{d x^{i}}{d x^{+}}\right) d x^{+} d x^{+}
$$

from which the einbein $h$ can be read off as

$$
h\left(x^{+}\right)=\dot{x}^{-}-\dot{x}^{i} \dot{x}^{i} \equiv v^{-}-\mathbf{v}_{\perp}^{2} .
$$

Note that velocities are dimensionless, so that despite appearance the result is consistent (if you do not like it as it stands, just insert the appropriate factors of $c)$. 
The Hamiltonian is obtained by solving the constraint $p^{+} p^{-}-p_{\perp}^{2}-m^{2}=0$, which is now linear in $p^{-}$. The result is

$$
H_{x^{+}}=n \cdot p=p^{-} / 2=\frac{p_{\perp}^{2}+m^{2}}{2 p^{+}} .
$$

Let me reemphasize that this Hamiltonian does not contain a square root as already pointed out by Dirac. However, now it is crucial that the FP operator is nonvanishing,

$$
\mathrm{FP}=-2 N \cdot p=-2 p^{+} \neq 0 .
$$

While this is always true for massive particles, it is violated for massless 'leftmovers', i.e. for particles travelling in the negative $z$-direction at the speed of light. In this case, we have a 'Gribov problem' (Gribov 1978), as the particles move within our gauge-fixing hyperplane, $x^{+}=0$. We will return to this issue later on.

For massive particles, the dynamics is consistently generated by means of the Poisson brackets

$$
v^{-}=\dot{x}^{-}=\left\{x^{-}, H_{x^{+}}\right\}=\frac{p^{-}}{p^{+}}, \quad v^{i}=\dot{x}^{i}=\left\{x^{i}, H_{x^{+}}\right\}=\frac{p^{i}}{p^{+}} .
$$

Note finally, that the Hamiltonian (74) is not the normal projection $N \cdot p$ of the momentum, because $N \cdot p$ lies within $\Sigma$ and thus corresponds to a kinematical direction.

As for the instant form, the light-cone representation of the Poincaré generators can be obtained by solving the constraint $p^{2}=m^{2}$ for $p^{-}$, inserting the result into the elementary representation (54) of the generators and setting $x^{+}=0$. The kinematical generators are

$$
\begin{aligned}
P^{i} & =p^{i}, \quad P^{+}=p^{+}, \\
M^{+i} & =-x^{i} p^{+}, \quad M^{12}=x^{1} p^{2}-x^{2} p^{1}, \quad M^{+-}=-x^{-} p^{+} .
\end{aligned}
$$

They correspond to transverse and longitudinal translations within $\Sigma\left(P^{i}\right.$ and $P^{+}$, respectively), transverse boosts and rotations $\left(M^{+i}\right)$, rotations around the $z$-axis $\left(M^{12}\right)$ and boosts $(!)$ in the $z$-direction $\left(M^{+-}\right)$. The latter will be further analysed in a moment. We thus have found seven kinematical generators, so that the front form leads to the largest stability group among Dirac's forms of dynamics (cf. Table 1).

The dynamical generators are given by

$$
P^{-}=\frac{p_{\perp}^{2}+m^{2}}{p^{+}}, \quad M^{-i}=x^{-} p^{i}-x^{i} p^{-} .
$$

As expected, the $M^{-i}$ depend on the Hamiltonian, $p^{-}$. If we now consider rotations around the $x-$ or $y$-axis, generated by

$$
\begin{aligned}
& L^{1}=M^{23}=\frac{1}{2}\left(M^{2+}-M^{2-}\right), \\
& L^{2}=M^{31}=\frac{1}{2}\left(M^{+1}-M^{-1}\right),
\end{aligned}
$$


we note that they correspond to dynamical operations due to the appearance of $M^{-i}$. This leads to the notorious 'problem of angular momentum' within the front form, see e.g. (Fuda 1990). Except for the free theory, it is very hard to write down states with good angular momentum as diagonalizing $\mathbf{L}^{2}$ is as difficult as solving the Schrödinger equation. A similar problem arises for parity. This exchanges light-cone space and time and thus also becomes dynamical (Burkardt 1996). For the kinematical component of the angular momentum, $L_{z}=M^{12}$, these difficulties do not arise.

Consider now the following boost in $z$-direction with rapidity $\omega$ written in instant-form coordinates,

$$
\begin{aligned}
& t \rightarrow t \cosh \omega+z \sinh \omega \\
& z \rightarrow t \sinh \omega+z \cosh \omega .
\end{aligned}
$$

As stated before, such a boost mixes space and time coordinates $z$ and $t$. If we add and subtract these equations, we obtain the action of the boost for the front form,

$$
\begin{aligned}
& x^{+} \rightarrow e^{\omega} x^{+}, \\
& x^{-} \rightarrow e^{-\omega} x^{-} .
\end{aligned}
$$

We thus find the important result that a boost in $z$-direction does not mix light-cone space and time but rather rescales the coordinates! Note that $x^{+}$and $x^{-}$are rescaled inversely with respect to each other. The scaling factor can be written as

$$
e^{\omega}=\sqrt{\frac{1-v}{1+v}}
$$

if the rapidity $\omega$ is defined in the usual manner in terms of the boost velocity $v$, $\tanh \omega=v$. One should note in particular, that one has the fixed point hypersurface $\Sigma: x^{+}=0$ which is mapped onto itself, so that the relevant generator, $M^{+-}=2 M^{30}=-2 K^{3}$, is kinematical, in agreeement with (77). However, we see explicitly that this is no longer true for $x^{+} \neq 0$, where we get a rescaling of $x^{+}$. Stated differently, the transformation to light-cone coordinates diagonalizes the boosts in $z$-direction. Therefore, the behavior under such boosts becomes especially simple. A pedagogical discussion and some elementary applications can be found in (Parker and Schmieg 1970).

We are actually more interested in the transformation properties of the momenta, as these, being Poincaré generators, are more fundamental quantities than the coordinates, in particular in the quantum theory (Leutwyler and Stern 1978). As $P^{\mu}$ transforms as a four-vector we just have to replace $x^{\mu}$ by $P^{\mu}$ in the boost transformations $(83,84)$ and obtain,

$$
\begin{aligned}
& P^{+} \rightarrow e^{\omega} P^{+}, \\
& P^{-} \rightarrow e^{-\omega} P^{-}, .
\end{aligned}
$$


We remark that $P^{+}=0$ is a fixed point under longitudinal boosts. In quantum field theory, it corresponds to the vacuum. For the transverse momentum, $P^{i}$, one finds a transformation law reminiscent of a Galilei boost,

$$
P^{i} \rightarrow P^{i}+v^{i} P^{+} .
$$

In this identity, describing the action of $M^{+i}$, longitudinal and transverse momenta (which are both kinematical) get mixed.

We can now ask the question how to boost from $\left(P^{+}, P^{i}\right)$ to momenta $\left(Q^{+}, Q^{i}\right)$. This can be done by fixing the boost parameters $\omega$ and $v^{i}$ as

$$
\omega=-\log \frac{Q^{+}}{P^{+}}, \quad v^{i}=\frac{Q^{i}-P^{i}}{P^{+}} .
$$

Obviously, this is only possible for $P^{+} \neq 0$. We emphasize that in the construction above there is no dynamics involved. For the quantum theory, this means that we can build states of arbitrary light-cone momenta with very little effort. All we have to do is applying some kinematical boost operators. The simple behavior of light-cone momenta under boosts will be important for the discussion of bound states in Section 4.

The similarity between (88) and Galilei boosts is not accidental. This is exhibited by the following subalgebra of the light-cone Poincaré algebra (Susskind 1968; Bardakci and Halpern 1968). Consider the Poisson bracket relations of the seven generators $P^{\mu}, M^{12}, M^{+i}$,

$$
\begin{aligned}
\left\{M^{12}, M^{+i}\right\} & =\epsilon^{i j} M^{+j} \\
\left\{M^{12}, P^{i}\right\} & =\epsilon^{i j} P^{j} \\
\left\{M^{+i}, P^{-}\right\} & =-2 P^{i} \\
\left\{M^{+i}, P^{j}\right\} & =-\delta^{i j} P^{+}
\end{aligned}
$$

All other brackets of these generators vanish. Compare now with the twodimensional Galilei group. Its generators (for a free particle of mass $\mu$ ) are: two momenta $k^{i}$, one angular momentum $L=\epsilon^{i j} x^{i} k^{j}$, two Galilei boosts $G^{i}=\mu x^{i}$, the Hamiltonian $H=k^{i} k^{i} / 2 \mu$ and the mass $\mu$, which is the Casimir generator. Upon using $\left\{x^{i}, k^{j}\right\}=\delta^{i j}$ and identifying $P^{i} \leftrightarrow k^{i}, M^{12} \leftrightarrow L, M^{+i} \leftrightarrow$ $-2 G^{i}, P^{+} \leftrightarrow 2 \mu$ and $P^{-} \leftrightarrow H$, one easily finds that (90) forms a subalgebra of the Poincaré algebra which is isomorphic to the Lie algebra of the two-dimensional Galilei group. (A second isomorphic subalgebra is obtained via identifying $M^{-i} \leftrightarrow 2 G^{i}$ and exchanging $P^{+}$with $P^{-}$.) The first two identities in (90), for instance, state that $M^{+i}$ and $P^{i}$ transform as ordinary twodimensional vectors. $P^{+}$can be interpreted as a variable Galilei mass which is also obvious from the nonrelativistic appearance of the light-cone Hamiltonian, $P^{-}=\left(P_{\perp}^{2}+m^{2}\right) / P^{+}$and the Galilei boost (88).

One thus expects that light-cone kinematics will partly show a nonrelativistic behavior which is associated with the transverse dimensions and governed by the two-dimensional Galilei group. This expectation is indeed realized and leads, 
for instance, to a separation of center-of-mass and relative dynamics in multiparticle systems. This will be discussed at length in the beginning of Section 4 .

So far, our discussion of the Poincaré algebra was restricted to the free case. With the inclusion of interactions, one expects all dynamical Poincaré generators to differ from their free counterpart by some 'potential' term $W$. This has already been pointed out by (Dirac 1949), who also stated that finding potentials which are consistent with the commutation relations of the Poincaré algebra is the "real difficulty in the construction of a theory of a relativistic dynamical system" with a fixed number of particles.

It has turned out, however, that Poincaré invariance alone is not sufficient to guarantee a reasonable Hamiltonian formulation. There are no-go theorems both for the instant (Leutwyler 1965) and the front form (Jaen et al. 1984), which state that the inclusion of any potential into the Poincaré generators, even if consistent with the commutation relations, spoils relativistic covariance. The latter is a stronger requirement as it enforces particular transformation laws for the particle coordinates. Thus, covariance imposes rather severe restrictions on the dynamical system (Leutwyler and Stern 1978).

The physical reason for these problems is that potentials imply an instantaneous interaction-at-a-distance which is in conflict with the existence of a limiting velocity and retardation effects. Relativistic causality is thus violated. This is equivalently obvious from the fact that a fixed number of particles is in conflict with the necessity of particle creation and annihilation and the appearance of antiparticles.

Nevertheless, with considerable effort, it is possible to construct dynamical quantum systems with a fixed number of constituents which are consistent with the requirements of Poincaré invariance and relativistic covariance (Leutwyler and Stern 1978; Sokolov and Shatnii 1979; Coester and Polyzou 1982).

At this point one might finally ask whether the different forms of relativistic dynamics are physically equivalent. From the point of view that different time choices correspond to different gauge fixings it is clear that equivalence must hold. After all, we are just dealing with different coordinate systems. People have tried to make this equivalence more explicit by working with coordinates which smoothly interpolate between the instant and the front form (Prokhvatilov and Franke 1988; Prokhvatilov and Franke 1989; Lenz et al. 1991; Hornbostel 1992). In the context of relativistic quantum mechanics, it has been shown that the Poincaré generators for different forms are unitarily equivalent (Sokolov and Shatnii 1979).

We are, however, more interested in what might be called a 'top-down approach'. Our aim is to describe few-body systems not within quantum mechanics but quantum field theory to which we now turn. 


\section{Light-Cone Quantization of Fields}

\subsection{Construction of the Poincaré Generators}

We want to derive the representation of the Poincaré generators within field theory and their dependence on the hypersurface $\Sigma$ chosen to define the time evolution. To this end we follow (Fubini et al. 1973) and describe the hypersurface mathematically through the equation

$$
\Sigma: F(x)=\tau .
$$

The surface element on $\Sigma$ is implicitly defined via

$$
\int_{\Sigma} d \sigma_{\mu} u(x)=\int d^{4} x N^{\mu} \delta(F(x)-\tau) u(x),
$$

where, as before, $N^{\mu}=\partial^{\mu} F(x)$ is the normal on $\Sigma$ and $u$ some integrable function. We will write this expression symbolically as

$$
d \sigma_{\mu}=d^{4} x N^{\mu} \delta(F(x)-\tau) .
$$

The central object of this subsection will be the energy-momentum tensor,

$$
T^{\mu \nu}=\frac{\partial \mathcal{L}}{\partial\left(\partial_{\mu} \phi\right)} \partial^{\nu} \phi-g^{\mu \nu} \mathcal{L},
$$

with $\mathcal{L}$ being the Lagrangian depending on fields that are collectively denoted by $\phi$. With the help of the energy-momentum tensor (94) we can define a generator

$$
A[f]=\int_{\Sigma} d \sigma_{\mu} f_{\nu}(x) T^{\mu \nu}(x),
$$

where $A$ and $f$ can be tensorial quantities carrying dummy indices $\alpha, \beta, \ldots$ which we have suppressed. $A[f]$ generates the infinitesimal transformations

$$
\begin{aligned}
\delta_{f} B(x) & =f_{\mu}(x) \partial^{\mu} B(x), \\
\delta_{f} x^{\mu} & =f^{\mu}(x),
\end{aligned}
$$

where $f$ is now understood as being infinitesimal. In the same way as for a finite number of degrees of freedom, the generator $A$ is called kinematical, if it leaves $\Sigma$ invariant, that is,

$$
\delta_{f} F=f_{\mu} \partial^{\mu} F=f \cdot N=0 .
$$

Otherwise, $A$ is dynamical. With the energy-momentum tensor $T^{\mu \nu}$ at hand, we can easily show that kinematical generators are interaction independent. We decompose $T^{\mu \nu}$,

$$
T^{\mu \nu}=T_{0}^{\mu \nu}-g^{\mu \nu} \mathcal{L}_{\text {int }},
$$


into a free part $T_{0}^{\mu \nu}=T^{\mu \nu}(g=0), g$ denoting the coupling, and an interacting part (we exclude the case of derivative coupling). If $A$ is kinematical, we have from $(93,95,98)$,

$$
A_{\mathrm{int}}[f]=-\int d^{4} x \delta(F-\tau) \mathcal{L}_{\mathrm{int}} f^{\mu} \partial_{\mu} F=-\int d^{4} x \delta(F-\tau) \mathcal{L}_{\mathrm{int}} \delta_{f} F=0,
$$

which indeed shows that $A$ does not depend on the interaction. Dynamical operators, on the other hand, will contain interaction dependent pieces. Of course, we are particularly interested in the Poincaré generators, $P^{\alpha}$ and $M^{\alpha \beta}$. They correspond to the choices $f_{\mu}^{\alpha}=g_{\mu}^{\alpha}$ and $f_{\mu}^{\alpha \beta}=x^{\alpha} g_{\mu}^{\beta}-x^{\beta} g_{\mu}^{\alpha}$, respectively, so that, from (95), they are given in terms of $T^{\mu \nu}$ as

$$
\begin{aligned}
P^{\alpha} & =\int_{\Sigma} d \sigma_{\mu} T^{\mu \alpha}, \\
M^{\alpha \beta} & =\int_{\Sigma} d \sigma_{\mu}\left(x^{\alpha} T^{\mu \beta}-x^{\beta} T^{\mu \alpha}\right) .
\end{aligned}
$$

From (98) it is easily seen that the Poincare generators defined in $(101,102)$ act on $F(x)=\tau$ exactly as described in (57). The remarks of Section 2 on the kinematical or dynamical nature of the generators in the different forms are therefore equally valid in quantum field theory.

Let us first discuss the instant form. We recall the hypersurface of equal time, $\Sigma: F(x) \equiv N \cdot x \equiv x^{0}=\tau$, which leads to a surface element

$$
d \sigma^{\mu}=d^{4} x N^{\mu} \delta\left(x^{0}-\tau\right), \quad N^{\mu}=(1, \mathbf{0}) .
$$

Using $(101,102)$, the Poincaré generators are obtained as

$$
\begin{aligned}
P^{\mu} & =\int_{\Sigma} d^{3} x T^{0 \mu}, \\
M^{\mu \nu} & =\int_{\Sigma} d^{3} x\left(x^{\mu} T^{0 \nu}-x^{\nu} T^{0 \mu}\right) .
\end{aligned}
$$

For the front form, quantization hypersurface and surface element are given by

$$
\Sigma: F(x) \equiv N \cdot x \equiv x^{+}=\tau, \quad d \sigma^{\mu}=d^{4} x N^{\mu} \delta\left(x^{+}-\tau\right),
$$

where $N$ is the light-like four-vector of (70). In terms of $T^{\mu \nu}$, the Poincaré generators are

$$
\begin{aligned}
P^{\mu} & =\frac{1}{2} \int_{\Sigma} d x^{-} d^{2} x_{\perp} T^{+\mu}, \\
M^{\mu \nu} & =\frac{1}{2} \int_{\Sigma} d x^{-} d^{2} x_{\perp}\left(x^{\mu} T^{+\nu}-x^{\nu} T^{+\mu}\right) .
\end{aligned}
$$

The somewhat peculiar factor $1 / 2$ is the Jacobian which arises upon transforming to light-cone coordinates. 


\subsection{Schwinger's (Quantum) Action Principle}

Our next task is to actually quantize the fields on the hypersurfaces $\Sigma: \tau=$ $F(x)$ of equal time $\tau$. There is more than one possibility to do so, and we will explain a few of these. We begin with a method that is essentially due to Schwinger (Schwinger 1951; Schwinger 1953a; Schwinger 1953b). We define a four-momentum density

$$
\Pi^{\mu}=\frac{\partial \mathcal{L}}{\partial\left(\partial_{\mu} \phi\right)}
$$

so that the energy-momentum tensor $T^{\mu \nu}$ can be written as

$$
T^{\mu \nu}=\Pi^{\mu} \partial^{\nu} \phi-g^{\mu \nu} \mathcal{L} .
$$

In some sense, this can be viewed as a covariant generalization of the usual Legendre transformation between Hamiltonian and Lagrangian. Using the normal $N^{\mu}$ of the hypersurface $\Sigma$, we define the canonical momentum (density) as the projection of $\Pi^{\mu}$,

$$
\pi \equiv N \cdot \Pi \text {. }
$$

Schwinger's action principle states that, upon variation, the action $S=\int d^{d} x \mathcal{L}$ changes at most by a surface term which (if $\Sigma$ is not varied, i.e. $\delta x^{\mu}=0$ ) is given by

$$
\delta G(\tau)=\int_{\Sigma} d \sigma_{\mu} \Pi^{\mu} \delta \phi=\int d^{d} x \delta(\tau-F) \pi \delta \phi .
$$

The quantity $\delta G$ is interpreted as the generator of field transformations, so that we have

$$
\delta \phi=\{\phi, \delta G\},
$$

in case that $\Sigma$ is entirely space-like (with time-like normal) (Schwinger 1951; Schwinger 1953a). We note in passing that the generator $\delta G$ is a field theoretic generalization of the canonical one-form $d G \equiv p_{i} d q^{i}$ used in analytical mechanics.

As in the preceding section we have to distinguish two cases depending on whether the normal vector $N$ is time-like or space-like. For time-like $N$, the associated hypersurface is space-like. The basic example for this case is the instant form, to which we immediately specialize. The canonical momentum density is given by the velocity, $\pi=\dot{\phi}$, and the Lagrangian is quadratic in $\dot{\phi}$. The canonical Poisson bracket is derived from Schwinger's action principle using (113),

$$
\begin{aligned}
\delta \phi(x) & =\{\phi(x), \delta G(\tau)\} \\
& =\int d y^{0} \int d^{3} y \delta\left(x^{0}-y^{0}\right)\{\phi(x), \pi(y)\} \delta \phi(y) \\
& =\left.\int d^{3} y\{\phi(x), \phi(y)\} \delta \phi(y)\right|_{x^{0}=y^{0}=\tau} .
\end{aligned}
$$


The canonical Poisson bracket, therefore, must be

$$
\{\phi(x), \phi(y)\}_{x, y \in \Sigma}=\delta^{3}(\mathbf{x}-\mathbf{y}),
$$

which, of course, is the standard result. The second case, $N$ light-like, corresponds to the front form. With minor modifications, Schwinger's approach can also be used here, resulting in what is interchangably called light-cone, lightfront or null-plane quantization. The canonical light-cone momentum is

$$
\pi=N \cdot \Pi=N \cdot \partial \phi=\partial^{+} \phi \equiv 2 \frac{\partial}{\partial x^{-}} \phi,
$$

which is peculiar to the extent that it does not involve a (light-cone) time derivative. Therefore, $\pi$ is a dependent quantity which does not provide additional information, being known on $\Sigma$ when the field is known there. Thus, $\pi$ is merely an abbreviation for $\partial^{+} \phi$ which is a spatial derivative. Again, the reason is that the normal $N^{\mu}$ of the null-plane $\Sigma$ lies within $\Sigma$. This leads to the important consequence that the light-cone Lagrangian is linear in the velocity $\partial^{-} \phi$, or, put differently, that light-cone field theories are first-order systems. As a result, $\phi$ and $\partial^{+} \phi$ have to be treated on the same footing within Schwinger's approach which leads to an additional factor $1 / 2$ compared to (113),

$$
\frac{1}{2} \delta \phi=\{\phi, \delta G\},
$$

with a front-form generator

$$
\delta G\left(x^{+}\right)=\frac{1}{2} \int_{\Sigma} d x^{-} d^{2} x_{\perp} \partial^{+} \phi \delta \phi .
$$

The appearance of the peculiar factor $1 / 2$ in (117) has been discussed at length by (Schwinger 1953b) - see also (Chang et al. 1973). Roughly speaking it stems from the fact that the independent field content within the front form is only one half of that in the instant form. The factor $1 / 2$ cancels the light-cone Jacobian $J=1 / 2$ in (118), so that we are left with the Poisson bracket,

$$
\{\phi(x), \pi(y)\}_{x, y \in \Sigma}=\delta\left(x^{-}-y^{-}\right) \delta^{2}\left(\mathbf{x}_{\perp}-\mathbf{y}_{\perp}\right) .
$$

As usual, commutators are inferred from Poisson brackets by invoking Dirac's correspondence principle, that is, by replacing the bracket by $i$ times the commutator. For arbitrary classical observables, $A, B$, this means explicitly,

$$
[\hat{A}, \hat{B}]=i\{\widehat{A, B}\} \text {. }
$$

We do not address the question of operator-ordering ambiguities at this point, as these will not be an issue in the applications to be discussed later on. One should, however, be aware of this problem, as it indeed can arise within the framework of light-cone quantization (Heinzl et al. 1996).

Using (120), the bracket (119) leads to the following commutator,

$$
[\phi(x), \pi(y)]_{x^{+}=y^{+}=\tau}=i \delta\left(x^{-}-y^{-}\right) \delta^{2}\left(\mathbf{x}_{\perp}-\mathbf{y}_{\perp}\right)
$$


As the independent quantities are the fields themselves, we invert the derivative $\partial^{+}$and obtain the more fundamental commutator

$$
[\phi(x), \phi(y)]_{x^{+}=y^{+}=\tau}=-\frac{i}{4} \operatorname{sgn}\left(x^{-}-y^{-}\right) \delta^{2}\left(\mathbf{x}_{\perp}-\mathbf{y}_{\perp}\right) .
$$

In deriving (122) we have chosen the anti-symmetric Green function $\operatorname{sgn}\left(x^{-}\right)$ satisfying

$$
\frac{\partial}{\partial x^{-}} \operatorname{sgn}\left(x^{-}\right)=2 \delta\left(x^{-}\right),
$$

so that (121) is reobtained upon differentiating (122) with respect to $y^{-}$. We will see later that the field commutator (122) can be derived directly within Schwinger's method. Before that, however, let us study the relation between the choice of initializing hypersurfaces, the problem of field quantization and the solutions of the dynamical equations.

\subsection{Quantization as an Initial- and/or Boundary-Value Problem}

As a prototype field theory we consider a massive scalar field $\phi$ in $1+1$ dimensions. Its dynamics is encoded in the action

$$
S[\phi]=\int d^{2} x \mathcal{L}=\int d^{2} x\left(\frac{1}{2} \partial_{\mu} \phi \partial^{\mu} \phi-\frac{1}{2} m^{2} \phi^{2}-\mathcal{V}[\phi]\right),
$$

where $\mathcal{V}$ is some interaction term like e.g. $\lambda \phi^{4}$ and $\mathcal{L}=\mathcal{L}_{0}+\mathcal{V}$. By varying the free action in the standard way we obtain

$$
\delta S=\int_{\partial M} d \sigma_{\mu} \Pi^{\mu} \delta \phi+\int_{M}\left[\frac{\partial \mathcal{L}_{0}}{\partial \phi}-\partial_{\mu} \frac{\partial \mathcal{L}_{0}}{\partial\left(\partial_{\mu} \phi\right)}\right] \delta \phi .
$$

If we do not vary on the boundary of our integration region $M,\left.\delta \phi\right|_{\partial M}=0$, the surface term in $\delta S$ (which is closely related to $\delta G$ from (112)), vanishes and we end up with the (massive) Klein-Gordon equation in $1+1$ dimensions,

$$
\left(\square+m^{2}\right) \phi=0 .
$$

In this subsection, we will solve this equation by specifying initial and/or boundary conditions for the scalar field $\phi$ on different hypersurfaces $\Sigma$. In addition, we will clarify the relation between the associated initial value problems and the determination of 'equal-time' commutators.

It may look rather trivial to consider just the free theory, but this is not entirely true. Let us analyze what quantization of a field theory means in the light of the different forms of relativistic dynamics. One specifies canonical commutators like $[\phi(x), \phi(y)]_{x, y \in \Sigma}$, where the hypersurface $\Sigma: \tau=$ const defines the evolution parameter $\tau$. As both $x$ and $y$ lie in $\Sigma$, the commutator is evaluated at 'equal time', which implies that it is a kinematical quantity. Therefore, it is the same for the free and the interacting theory. 
Now, if $\phi$ is a free field, the commutator,

$$
[\phi(x), \phi(0)]=i \Delta(x),
$$

is exactly known: it is the Pauli-Jordan or Schwinger function $\Delta$ (Jordan and Pauli 1928; Schwinger 1949) which is a special solution of the Klein-Gordon equation (126). It can be obtained directly from the action in a covariant manner as a Peierls bracket (Peierls 1952; DeWitt 1984). Alternatively, one can find it by evaluating the Fourier integral,

$$
\begin{aligned}
\Delta(x) & =-\frac{i}{2 \pi} \int d^{2} p \delta\left(p^{2}-m^{2}\right) \operatorname{sgn}\left(p^{0}\right) e^{-i p \cdot x} \\
& =-\frac{1}{2} \operatorname{sgn}\left(x^{0}\right) \theta\left(x^{2}\right) J_{0}\left(m \sqrt{x^{2}}\right) \\
& =-\frac{1}{4}\left[\operatorname{sgn}\left(x^{+}\right)+\operatorname{sgn}\left(x^{-}\right)\right] J_{0}\left(m \sqrt{x^{+} x^{-}}\right),
\end{aligned}
$$

where I have given both the instant and front form representation (Heinzl and Werner 1994). We note that $\Delta$ is antisymmetric, $\Delta(x)=-\Delta(-x)$ and Lorentz invariant (under proper orthochronous transformations). Most important, it is causal, i.e. it vanishes outside the light-cone, $x^{2}<0$ (see Fig. 2).

Fig. 2. The Pauli-Jordan function as a function of $T=m x^{0} / 2$ and $X=m x^{1} / 2$. It vanishes outside the light-cone and oscillates inside.

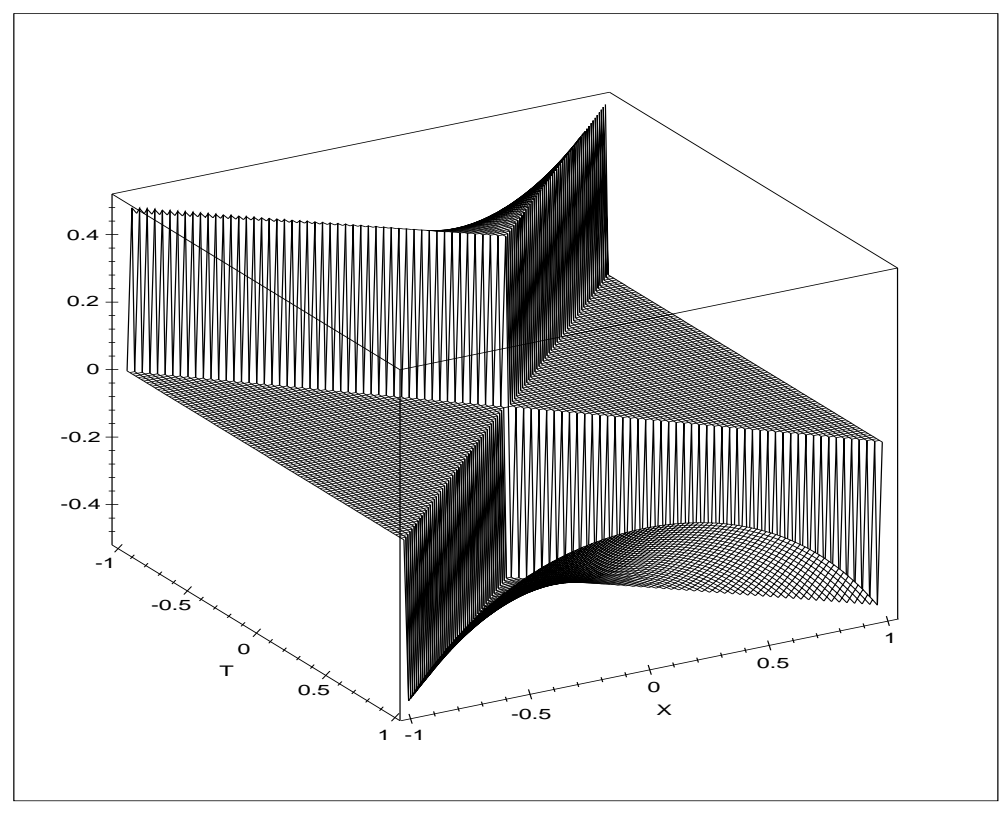


If $\phi$ is an interacting field, causality, of course, must still hold. If $x$ and $y$ are space-like with respect to each other, the commutator thus still vanishes,

$$
[\phi(x), \phi(y)]_{(x-y)^{2}<0}=0 .
$$

This expresses the fact that fields which are separated by a space-like distance cannot communicate with each other. For the front form, with the hypersurface $\Sigma: x^{+}=0,(129)$ cannot be used to obtain the canonical commutators: In $1+1$ dimensions, $\Sigma$ is part of the light-cone and therefore entirely light-like. In higher dimensions, $\Sigma$ still contains light-like directions namely where $x^{-}=\mathbf{x}_{\perp}=0$. For this reason, the light-front commutator (122) of two free fields does not vanish identically.

Let me now discuss the explicit relation between the choice of equal-time commutators and the classical initial/boundary for the Klein-Gordon equation. Three examples are of interest.

Cauchy Data: Instant Form. Conventional quantization on a space-like surface (based on the instant form) corresponds to a Cauchy problem: if one specifies the field $\phi$ and its time derivative $\dot{\phi}$ on $\Sigma: x^{0}=t=0$,

$$
\begin{aligned}
& \phi(t=0, x)=f(x), \\
& \dot{\phi}(t=0, x)=g(x),
\end{aligned}
$$

where the functions $f$ and $g$ denote the initial data (depending on $x \equiv x^{1}$ ), the solution of the Klein-Gordon equation is uniquely determined. This can be checked by considering the Taylor expansion around $(t, x) \in \Sigma$, i.e. $t=0$,

$$
\phi(t, x)=\phi(0, x)+t \dot{\phi}(0, x)+\frac{1}{2} t^{2} \ddot{\phi}(0, x)+\ldots,
$$

with the overdot denoting the time derivative. From this we see that one has to know all time derivatives of $\phi$ on $\Sigma$ once the data $f, g$ are given. If we calculate these,

$$
\begin{aligned}
\phi & =f, \\
\dot{\phi} & =g, \\
\ddot{\phi} & =\phi^{\prime \prime}-m^{2} \phi=f^{\prime \prime}-m^{2} f, \\
\frac{\partial^{3} \phi}{\partial t^{3}} & =\dot{\phi}^{\prime \prime}-m^{2} \dot{\phi}=g^{\prime \prime}-m^{2} g, \\
\vdots & .
\end{aligned}
$$

we find that indeed all time derivatives are given in terms of $f$ and $g$ and their known spatial derivatives, denoted by the prime. In the last two identities, we have made use of the equation of motion. As a result, we see that the Cauchy problem is well posed: the solution of the Klein-Gordon equation is uniquely determined by the data on $\Sigma$. 
Upon quantization, this translates into the fact that the Fock operators can be expressed in terms of the data,

$$
a\left(p^{1}\right)=\int d x^{1} e^{-i p^{1} x^{1}}\left[\omega_{p} \phi\left(x^{0}=0, x^{1}\right)+i \dot{\phi}\left(x^{0}=0, x^{1}\right)\right],
$$

with $\omega_{p}=\left(p_{1}^{2}+m^{2}\right)^{1 / 2}$. In addition, the canonical commutators can be viewed as the Cauchy data for the Pauli-Jordan function $\Delta$,

$$
\begin{aligned}
& {[\phi(x), \phi(0)]_{x^{0}=0}=\left.i \Delta(x)\right|_{x^{0}=0}=0,} \\
& {[\dot{\phi}(x), \phi(0)]_{x^{0}=0}=\left.i \dot{\Delta}(x)\right|_{x^{0}=0}=-i \delta\left(x^{1}\right) .}
\end{aligned}
$$

As stated above, the vanishing of the commutator (135) is due to causality.

The Characteristic Initial-Value Problem. In the following I will perform an analogous discussion for the hypersurfaces $\Sigma: x^{ \pm}=$const, which, in $d=1+1$, constitute the entire light-cone, $x^{2}=0$. In Dirac's classification, the light-cone corresponds to a degenerate point form with parameter $a=0$ (see Table 1). One thus does not have transitivity as points on different 'legs' of the cone are not related by a kinematical operation. Still, it turns out that the associated initial-value problem is well posed (Neville and Rohrlich 1971; Rohrlich 1971)

The light-fronts $x^{ \pm}=0$ are characteristics of the Klein-Gordon equation (Domokos 1972). Therefore, one is dealing with a characteristic initial-value problem (Courant and Hilbert 1962), for which one has to provide the data

$$
\begin{aligned}
\phi\left(x^{+}=0, x^{-}\right) & =f\left(x^{-}\right), \\
\phi\left(x^{+}, x^{-}=0\right) & =g\left(x^{+}\right), \\
f\left(x^{-}=0\right) & =g\left(x^{+}=0\right),
\end{aligned}
$$

where the last identity is a continuity condition. Consistency is again checked by Taylor expanding, this time around $\left(x^{+}, x^{-}\right)=0$,

$$
\begin{aligned}
\partial^{+} \phi & =\partial^{+} f \equiv f^{\prime}, \\
\partial^{-} \phi & =\partial^{-} g \equiv \dot{g}, \\
\partial^{+} \partial^{-} \phi & =m^{2} \phi=m^{2} f=m^{2} g, \\
\partial^{+} \partial^{+} \phi & =f^{\prime \prime} \\
\partial^{-} \partial^{-} \phi & =\ddot{g} \\
\partial^{+} \partial^{+} \partial^{-} \phi & =m^{2} f^{\prime} \\
\partial^{-} \partial^{-} \partial^{+} \phi & =m^{2} \dot{g} \\
\vdots & .
\end{aligned}
$$

Whereever a factor of $m^{2}$ appears we have made use of the Klein-Gordon equation. We thus note that the data (together with their known derivatives) determine all partial derivatives of $\phi$ at the vertex of the cone, $\left(x^{+}, x^{-}\right)=0$. 
Intuitively, this corresponds to the fact that the information spreads from a source located at origin.

The characteristic initial-value problem amounts to quantization on two characteristics, $x^{ \pm}=0$, i.e., in $d=1+1$, really on the light cone, $x^{2}=0$. The following two independent commutators,

$$
[\phi(x), \phi(0)]_{x^{ \pm}=0}=\left.i \Delta(x)\right|_{x^{ \pm}=0}=-\frac{i}{4} \operatorname{sgn}\left(x^{\mp}\right),
$$

are then characteristic data for the Pauli-Jordan function. It turns out that, in case the field $\phi$ is massless, the above quantization procedure is the only consistent one (in $d=1+1$ ), if one wants to use light-like hypersurfaces (Bogolubov et al. 1990).

However, it is important to note that the characteristic initial-value problem does not correspond to light-cone quantization. One would need two Hamiltonians $P^{-}$and $P^{+}$, and, accordingly, two time parameters. This seems somewhat weird, to say the least, and will not be pursued any further.

Initial-Boundary-Data: Front-Form. In order to find the initial-value problem of the front form with a single time parameter $x^{+}$, let us naively try a straightforward analog of the Cauchy data and prescribe field and velocity on $\Sigma: x^{+}=0$

$$
\begin{aligned}
\phi\left(x^{+}=0, x^{-}\right) & =f\left(x^{-}\right), \\
\partial^{-} \phi\left(x^{+}=0, x^{-}\right) & =g\left(x^{-}\right) .
\end{aligned}
$$

It turns out, however, that this overdetermines the system. Namely, from the equation of motion

$$
\partial^{+} \partial^{-} \phi=m^{2} \phi
$$

it is actually possible to obtain the velocity $\partial^{-} \phi$ by inversion of the spatial derivative $\partial^{+}$,

$$
\partial^{-} \phi=m^{2}\left(\partial^{+}\right)^{-1} \phi=m^{2}\left(\partial^{+}\right)^{-1} f .
$$

The last identity holds on $\Sigma$ and implies that the data $g$ are unnecessary (and will even lead to an inconsistency) as the velocity is already determined by $f$. This is confirmed by the Taylor expansion on $\Sigma$,

$$
\begin{aligned}
\phi & =f, \\
\partial^{+} \phi & =\partial^{+} f, \\
\partial^{-} \phi & =m^{2}\left(\partial^{+}\right)^{-1} f, \\
\partial^{-} \partial^{-} \phi & =m^{2}\left(\partial^{+}\right)^{-1} \partial^{-} \phi=m^{4}\left(\partial^{+}\right)^{-2} f, \\
\vdots & .
\end{aligned}
$$

It thus seems that the front form requires only half of the data as compared to the instant form. This appearance, however, is deceptive. Note that we have to 
invert the differential operator $\partial^{+}$. The inverse is nothing but the Green function $G$ defined via

$$
\partial^{+} G\left(x^{-}\right)=\delta\left(x^{-}\right) .
$$

Clearly, this Green function is determined only up to a homogeneous solution $h$ satisfying

$$
\partial^{+} h=0,
$$

i.e. up to a zero mode $h=h\left(x^{+}\right)$of the operator $\partial^{+}$. Thus, in order to uniquely specify the Green function (147), we have to provide additional information in terms of boundary conditions. The standard choice is to demand antisymmetry in $x^{-}$, whence

$$
G\left(x^{-}\right)=\frac{1}{4} \operatorname{sgn}\left(x^{-}\right),
$$

which we have already used in (122) and (123). Before we discuss the physical reason for demanding antisymmetry, let us briefly go to momentum space where we replace $\partial^{+}$by $i p^{+}$. The equation (147) for the Green function becomes $i p^{+} G\left(p^{+}\right)=1$, which has the general solution

$$
G\left(p^{+}\right)=-i / p^{+}+h\left(p^{-}\right) \delta\left(p^{+}\right) .
$$

In this identity, $1 / p^{+}$has to be viewed as a distribution corresponding to an arbitrary regularization of the singular function $1 / p^{+}$(Gelfand and Shilov 1964). Any two regularizations differ by terms proportional to $\delta\left(p^{+}\right)$, i.e. a zero mode of $p^{+}$. Choosing an antisymmetric Green function uniquely yields a principal value prescription,

$$
i G\left(p^{+}\right)=\mathcal{P} \frac{1}{p^{+}}=\frac{1}{2}\left(\frac{1}{p^{+}+i \epsilon}+\frac{1}{p^{+}-i \epsilon}\right),
$$

which is the canonical regularization of $1 / p^{+}$.

Altogether we have seen that the front form corresponds to prescribing both initial and boundary conditions, so that one has a 'mixed' or initial-boundary value problem. What are the implications for quantization? We address this question by determining the Poisson brackets through the requirement that EulerLagrange and canonical equations should be equivalent. To this end we solve the Klein-Gordon equation (126) for the velocity $\dot{\phi} \equiv \partial \phi / \partial x^{+}$as in (145). This gives

$$
\dot{\phi}\left(x^{+}, x^{-}\right)=-\frac{m^{2}}{4} \int d y^{-} G\left(x^{-}, y^{-}\right) \phi\left(x^{+}, y^{-}\right),
$$

using the Green function $G$ from (149). The Hamiltonian equation of motion is given by the Poisson bracket with $H=\frac{1}{2} m^{2} \int d x^{-} \phi^{2}$,

$$
\dot{\phi}\left(x^{+}, x^{-}\right)=\frac{m^{2}}{2} \int d y^{-}\left\{\phi\left(x^{+}, x^{-}\right), \phi\left(x^{+}, y^{-}\right)\right\} \phi\left(x^{+}, y^{-}\right)
$$

with the bracket of the fields $\phi$ to be determined. Clearly, Euler-Lagrange and Hamiltonian equation of motion, (152) and (153) become equivalent if one identifies

$$
\left\{\phi\left(x^{+}, x^{-}\right), \phi\left(x^{+}, y^{-}\right)\right\} \equiv-\frac{1}{2} G\left(x^{-}, y^{-}\right) .
$$


We thus see that the fundamental Poisson bracket coincides with the Green function which, accordingly, justifies the requirement of antisymmetry. After quantization, (154) of course coincides with (122), the result from Schwinger's action principle, specialized to $d=1+1$,

$$
[\phi(x), \phi(0)]_{x^{+}=0}=\left.i \Delta(x)\right|_{x^{+}=0}=-\frac{i}{4} \operatorname{sgn}\left(x^{-}\right) .
$$

From the momentum space perspective,

$$
\left[\phi\left(p^{+}\right), \phi(0)\right]=\frac{i}{2} G\left(p^{+}\right)=\frac{1}{2} \mathcal{P} \frac{1}{p^{+}},
$$

we conclude that, technically, light-cone quantization is the inversion of the longitudinal momentum $p^{+}$and as such requires the specification of initial-boundary data. In some sense, this can also be viewed as an infrared regularization because one provides a prescription of dealing with a pole at vanishing longitudinal momentum, $p^{+}=0$. As is well known, a particularly nice way of regularizing in the infrared is to enclose the system under consideration in a finite spatial volume. This is the topic of the next subsection.

\subsection{DLCQ - Basics}

DLCQ is the acronym for 'discretized light-cone quantization', originally developed by (Maskawa and Yamawaki 1976; Pauli and Brodsky 1985a; Pauli and Brodsky $1985 \mathrm{~b})^{4}$. The physical system under consideration is enclosed in a finite volume with discrete momenta and prescribed boundary conditions in $x^{-}$. Recently, there has been renewed interest in this method in the context of string theory (Banks et al. 1997; Susskind 1997; Lunin and Pinsky 1999).

Our starting point is the Fourier representation for a solution of the KleinGordon equation (still in infinite volume),

$$
\begin{aligned}
\phi(x) & =\int \frac{d^{2} p}{2 \pi} \chi(p) \delta\left(p^{+} p^{-}-m^{2}\right) e^{-i p \cdot x} \\
& =\int \frac{d p^{+} d p^{-}}{4 \pi} \chi\left(p^{+}, p^{-}\right) \frac{1}{\left|p^{+}\right|} \delta\left(p^{-}-m^{2} / p^{+}\right) e^{-i p \cdot x} \\
& =\int \frac{d p^{+}}{4 \pi\left|p^{+}\right|} \chi\left(p^{+}, \hat{p}^{-}\right) e^{-i \hat{p} \cdot x} \\
& =\int_{0}^{\infty} \frac{d p^{+}}{4 \pi p^{+}}\left[\chi\left(p^{+}, \hat{p}^{-}\right) e^{-i \hat{p} \cdot x}+\chi\left(-p^{+},-\hat{p}^{-}\right) e^{i \hat{p} \cdot x}\right] \\
& \equiv \int \frac{d p^{+}}{4 \pi p^{+}} \theta\left(p^{+}\right)\left[a\left(p^{+}\right) e^{-i \hat{p} \cdot x}+a^{*}\left(p^{+}\right) e^{i \hat{p} \cdot x}\right] .
\end{aligned}
$$

The following remarks are in order: we have defined the on-shell energy, $\hat{p}^{-} \equiv$ $\mathrm{m}^{2} / \mathrm{p}^{+}$; contrary to the instant form, the integration over the positive and negative mass hyperboloid is achieved by a single delta function. Again, this is a

\footnotetext{
${ }^{4}$ For a nice overview of recent developments see (Hiller 2000).
} 
consequence of the linearity of the mass-shell constraint in $p^{-}$. The two branches of the mass-shell correspond to positive and negative values of $p^{+}$(and also $\hat{p}^{-}$), respectively. Associated with the two signs of the kinematical momentum $p^{+}$are the positive and negative frequency modes $a, a^{*}$, defined in such a way that their argument $p^{+}$is always positive (cf. the step function $\theta$ in the last line). This can equivalently be viewed as the reality condition

$$
a^{*}\left(p^{+}\right)=a\left(-p^{+}\right),
$$

as is obvious from the last step in the derivation (157). Upon quantization this implies that annihilation operators with negative longitudinal momentum $p^{+}$are actually creation operators for particles with positive $p^{+}$. The field commutator (155) is reproduced by demanding

$$
\left[a\left(k^{+}\right), a^{\dagger}\left(p^{+}\right)\right]=4 \pi p^{+} \delta\left(k^{+}-p^{+}\right) .
$$

As already indicated, DLCQ amounts to compactifying the spatial light-cone coordinate, $-L \leq x^{-} \leq L$, and imposing periodic boundary conditions for the fields,

$$
\phi\left(x^{+}, x^{-}=-L\right)=\phi\left(x^{+}, x^{-}=L\right),
$$

which are to hold for all light-cone times $x^{+}$. Space-time is thus endowed with the topology of a cylinder. This implies discrete longitudinal momenta, $k_{n}^{+}=$ $2 \pi n / L$, so that the Fock expansion (157) becomes

$$
\phi\left(x^{+}=0, x^{-}\right)=a_{0}+\sum_{n>0} \frac{1}{\sqrt{4 \pi n}}\left(a_{n} e^{-i n \pi x^{-} / L}+a_{n}^{*} e^{i n \pi x^{-} / L}\right),
$$

Note that we have allowed for a zero momentum mode $a_{0}$. We will see in a moment that it actually vanishes in the free theory. Plugging (161) into the free Lagrangian

$$
L_{0}[\phi]=\frac{1}{2} \int d x^{-}\left(\frac{1}{2} \partial^{+} \phi \partial^{-} \phi-\frac{1}{2} m^{2} \phi^{2}\right)
$$

we obtain (discarding a total time derivative)

$$
L_{0}\left[a_{n}, a_{0}\right]=-i \sum_{n>0} a_{n} \dot{a}_{n}^{*}-m^{2} L a_{0}^{2}-\sum_{n>0} \frac{m^{2} L}{4 \pi n} a_{n}^{*} a_{n} \equiv-i \sum_{n>0} a_{n} \dot{a}_{n}^{*}-H,
$$

with $H$ denoting the Hamiltonian and $\dot{a}_{n}^{*}=\partial a_{n}^{*} / \partial x^{+}$. From both representations (162) and (163) it is obvious that the light-cone Lagrangian is linear in the velocity ( $\partial^{-} \phi$ and $\dot{a}_{n}^{*}$, respectively). A particularly suited method for quantization in this case is the one of Faddeev and Jackiw for first order systems (Faddeev and Jackiw 1988; Jackiw 1993). It avoids many of the technicalities of the Dirac-Bergmann formalism and is in general more economic. It reduces phase-space right from the beginning as there are no 'primary constraints' introduced. The method is essentially equivalent to Schwinger's action principle, especially in the form presented in (Schwinger 1953b). For the case at hand, the 
method basically boils down to demanding equivalence of the Euler-Lagrange and Hamiltonian equations of motion (cf. last subsection).

The former are given by

$$
\begin{aligned}
-i \dot{a}_{n}+\frac{m^{2} L}{4 \pi n} a_{n} & =0, \\
2 m^{2} L a_{0} & =0 .
\end{aligned}
$$

The first equation, (164), is just the free Klein-Gordon equation which can be easily seen upon multiplying by $k_{n}^{+}$. The second identity, (165), is nondynamical and thus a constraint which states the absence of a zero mode for free fields, $a_{0}=0$.

The canonical equations are

$$
\dot{a}_{n}=\left\{a_{n}, H\right\}=\sum_{k>0} \frac{m^{2} L}{4 \pi k}\left\{a_{n}, a_{k}^{*}\right\} a_{k},
$$

which obviously coincides with (164) if the canonical bracket is

$$
\left\{a_{k}, a_{n}^{*}\right\}=-i \delta_{k n} .
$$

The constraint (165) is obtained by differentiating the Hamiltonian,

$$
\frac{\partial H}{\partial a_{0}}=2 m^{2} L a_{0}=0 \text {. }
$$

Let us briefly show that the approach presented above is equivalent to Schwinger's (Schwinger 1953b). From (163) we read off a generator

$$
\delta G=-i \sum_{n>0} a_{n} \delta a_{n}^{*}
$$

effecting the transformation

$$
\delta a_{n}^{*}=\left\{a_{n}^{*}, \delta G\right\}=-i \sum_{k>0}\left\{a_{n}^{*}, a_{k}\right\} \delta a_{k}^{*},
$$

which in turn implies the canonical bracket (167).

Quantization is performed as usual by employing the correspondence principle (120), so that, from (167), the elementary commutator is given by

$$
\left[a_{m}, a_{n}^{\dagger}\right]=\delta_{m n} .
$$

The Fock space expansion for the (free) scalar field $\phi$ thus becomes

$$
\phi\left(x^{+}=0, x^{-}\right)=\sum_{n>0} \frac{1}{\sqrt{4 \pi n}}\left(a_{n} e^{-i n \pi x^{-} / L}+a_{n}^{\dagger} e^{i n \pi x^{-} / L}\right),
$$


Like in the infinite-volume expression (157), the Fock 'measure' $1 / \sqrt{4 \pi n}$ does not involve any scale like the mass $m$ or the volume $L$. This is at variance with the analogous expansion in the instant form which reads

$$
\phi(x, t=0)=\frac{1}{\sqrt{2 L}} \sum_{n} \frac{1}{\sqrt{2\left(k_{n}^{2}+m^{2}\right)}}\left(a_{n} e^{i k_{n} x}+a_{n}^{\dagger} e^{-i k_{n} x}\right)
$$

where $-L \leq x \leq L, k_{n}=\pi n / L$, and $\left[a_{n}, a_{m}^{\dagger}\right]=\delta_{m n}$. Obviously, the 'measure' $\left(k_{n}^{2}+m^{2}\right)^{1 / 2}$ does depend on $m$ and $L$. We will discuss some consequences of this difference in Subsection 3.6.

We can use the results (171) and (172) to calculate the free field commutator at equal light-cone time $x^{+}$,

$$
[\phi(x), \phi(0)]_{x^{+}=0}=\sum_{n \neq 0} \frac{1}{4 \pi n} e^{-i n \pi x^{-} / L}=-\frac{i}{2}\left[\frac{1}{2} \operatorname{sgn}\left(x^{-}\right)-\frac{x^{-}}{2 L}\right] .
$$

This coincides with (155) up to a finite size correction given by the additional term $x^{-} / 2 L$. The effect of this term is two-fold. First, it makes the sign function periodic (in the interval $-L \leq x^{-} \leq L$ ), and second, it guarantees the absence of a zero mode which must hold according to (165), (168), and

$$
\int_{-L}^{L} d x^{-}[\phi(x), \phi(0)]_{x^{+}=0}=0 .
$$

One may equally think of this as the finite-volume analog of the principal value prescription.

The commutator (174) has originally been obtained in (Maskawa and Yamawaki 1976) using the Dirac-Bergmann algorithm for constrained systems. The Faddeev-Jackiw method, however, is much more economic and transparent. In particular, it makes clear that the basic canonical variables of a light-cone field theory are the Fock operators or their classical counterparts. The $a_{n}$ with, say, $-N \leq n \leq N$ in (161) can be viewed as defining a $(2 N+1)$-dimensional phase space. A phase space, however, should have even dimension. This is accomplished by choosing a polarization in terms of positions and momenta, here $a_{n}$ and $a_{n}^{\dagger}$, with $n>0$, and by the vanishing of the zero mode, $a_{0}=0$. It turns out that this vanishing is a peculiarity of the free theory as is discussed in (Maskawa and Yamawaki 1976; Wittman 1989; Heinzl et al. 1992).

At this point one should honestly state that the issue of zero modes is one of the unsolved problems of light-cone quantization ${ }^{5}$. The constraint equations for the zero modes are in general very hard to solve unless one has some small parameter like in perturbation theory (McCartor and Robertson 1992; Heinzl et al. 1996) or within a large- $N$ expansion (Borderies et al. 1993; Borderies et al. 1995). Using a path integral approach, it has recently been shown (Hellerman

\footnotetext{
${ }^{5}$ For an overview see e.g. Ch. 7 of (Brodsky, Pauli, and Pinsky 1998) and references therein.
} 
and Polchinski 1999) that integrating out the zero modes constitutes a strong coupling problem. There are speculations that this problem might be less severe if one goes beyond quantum field theory, i.e. in string or M-theory (Banks 1999).

In the last reference, the author also states that compactification in a lightlike direction "is close to a space with periodic time" and thus "weird", in view of possible 'grandfather paradoxes'. Therefore, the natural question arises whether DLCQ is actually consistent with causality.

\subsection{DLCQ - Causality}

In this subsection I will address the question under which circumstances compactification of 'space' is compatible with the requirements of causality. The presented results are based on recent work with N. Scheu and H. Kröger (Heinzl et al. 1999).

In (128) and (129) we have seen that the (infinite-volume) commutator of two scalar fields vanishes whenever their space-time arguments are separated by a space-like distance (cf. Fig. 2). As already mentioned, this is a manifestation of the principle of microcausality, which is the general statement that the commutator of any two observables $\mathcal{O}_{1}(x)$ and $\mathcal{O}_{2}(y)$ must vanish whenever their separation $x-y$ is space-like. Physically, this implies that measurements of the observables $\mathcal{O}_{1}$ and $\mathcal{O}_{2}$ performed at $x$ and $y$, do not interfere. Some consequences of this principle are the spin-statistics theorem, analyticity properties of Green functions leading to dispersion relations etc. (Streater and Wightman 1963).

Our starting point are the Fourier representations of the Pauli-Jordan function, both for the instant and front form (denoted IF and FF, respectively),

$$
\begin{gathered}
\text { IF: } \quad \Delta(x)=-\int \frac{d k^{1}}{2 \pi \omega_{k}} \sin (k \cdot x) \equiv \int d k^{1} I\left(k^{1}\right), \\
\text { FF: } \Delta(x)=-\int_{0}^{\infty} \frac{d k^{+}}{2 \pi k^{+}} \sin (k \cdot x) \equiv \int d k^{+} I\left(k^{+}\right) .
\end{gathered}
$$

Both integrals yield the same result (128) for the Pauli-Jordan function. Note, however, that the integrand $I\left(k^{+}\right)$is exploding and rapidly oscillating for $k^{+} \rightarrow 0$ (see Fig. 3) so that the finite result for the integral is due to sizable cancellations that occur upon integration.

To obtain the finite-volume representations for the commutator, one proceeds as in Subsection 3.4 by restricting the spatial coordinates, $-L \leq x^{1}, x^{-} \leq L$, and imposing periodic boundary conditions for the field $\phi$. Momenta become discrete, $k_{n}^{1} \equiv \pi n / L$, and $k_{n}^{+} \equiv 2 \pi n / L$. The finite-volume representations are defined by replacing the integrals (176) and (177) by the discrete sums,

$$
\Delta_{I F}(x) \equiv-\sum_{n=-N}^{N} \frac{1}{2 \omega_{n} L} \sin \left(k_{n} \cdot x\right),
$$


Fig. 3. The integrand $I\left(k^{+}\right)$.

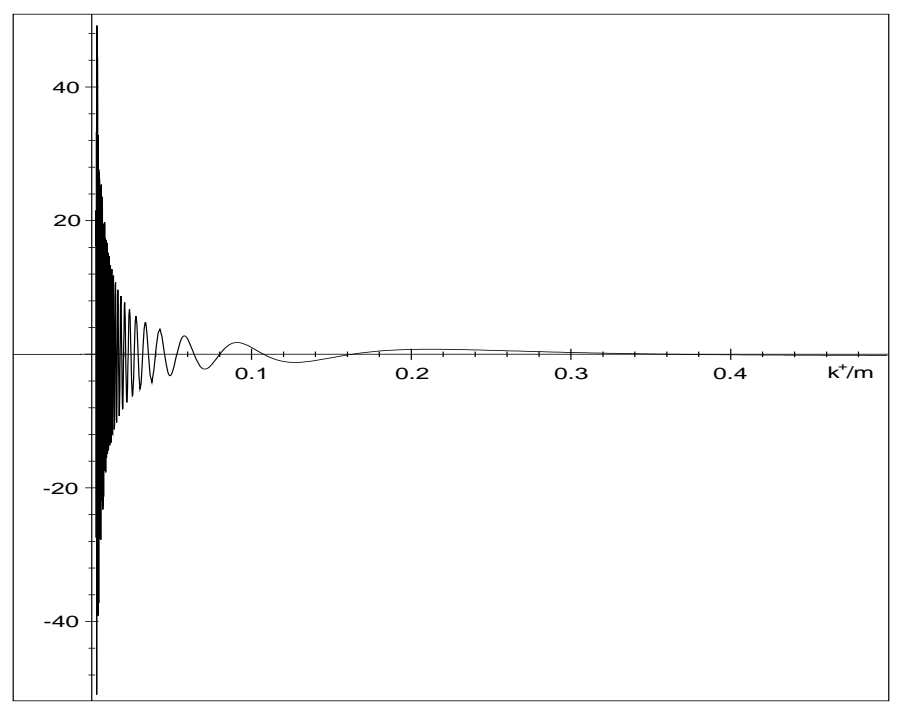

$$
\Delta_{F F}(x) \equiv-\sum_{n=1}^{N} \frac{1}{2 \pi n} \sin \left(k_{n} \cdot x\right)
$$

The on-shell energies for discrete momenta are defined as

$$
\omega_{n}=\left(n^{2} \pi^{2} / L^{2}+m^{2}\right)^{1 / 2} \quad \text { and } \quad \hat{k}_{n}^{-}=m^{2} L / 2 \pi n .
$$

For both functions, $\Delta_{I F}$ and $\Delta_{F F}$, the periodicity in $x^{1}$ and $x^{-}$, respectively, with periodicity length $2 L$, is obvious. The limit $N \rightarrow \infty$ is understood unless we perform numerical calculations where $N$ is kept finite.

The evaluation of the sums (178) and (179) is not straightforward. To gain some intuition, we evaluate them numerically beginning with the IF expression (178). The resulting $\Delta_{I F}$ is plotted in Fig. 4.

Upon inspection, one notes the following: Up to small oscillations stemming from the (unavoidable) Gibbs phenomenon, $\Delta_{I F}$ vanishes outside the lightcone $\left(\left|x^{0}\right|<\left|x^{1}\right|<L\right)$, and thus is causal even in finite volume. If we let the summation cutoff $N$ go to infinity, $\Delta_{I F}$ approaches the continuum Pauli-Jordan function $\Delta$ (for $-L<x^{0}, x^{1}<L$ ). There is a clear physical picture behind these observations. One can imagine a periodic array of sources located at the quantization hypersurface $x^{0}=0$ at points $x^{1}=2 L n$. These sources 'emit' spherical 'waves' into their own future LCs which start to overlap after time $x^{0}>L$. At this point the 'waves' emanating from the sources begin to interfere. Thus, the influence of the BC is felt only after a long time (as large as the 
Fig. 4. $\Delta_{I F}(X, T)$ as a function of $X=x^{1} / 2 L . T=X^{0} / 2 L=0.2, m L=1, N=50$.

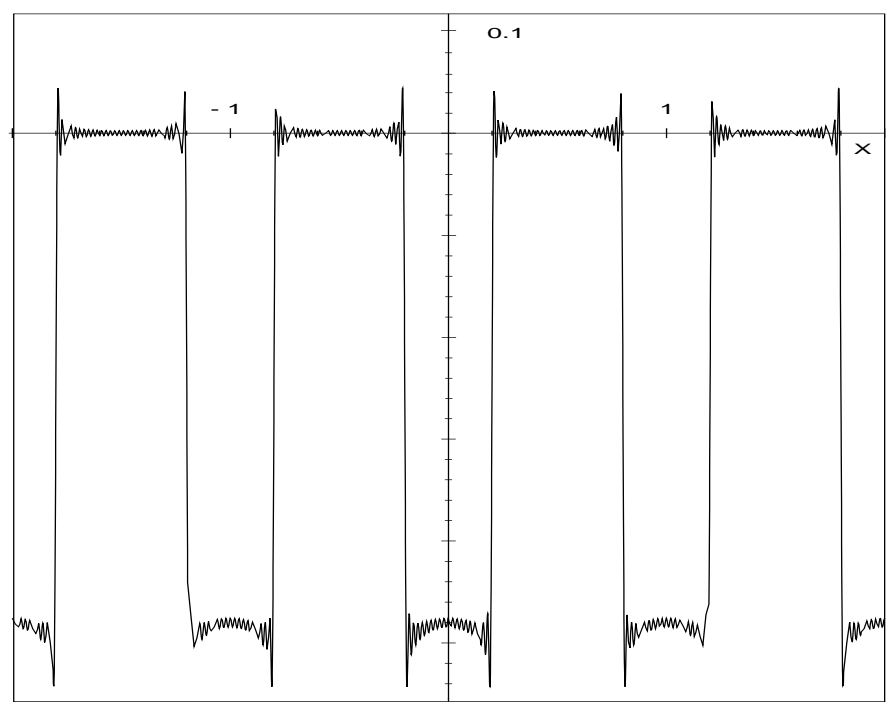

spatial extension $L$ of the system). This picture can be confirmed analytically. An application of the Poisson resummation formula yields

$$
\Delta_{I F}(x)=\sum_{n} \Delta\left(x^{0}, x^{1}+2 L n\right),
$$

i.e. a periodic array of $\Delta$ 's which are nonoverlapping as long as $x^{0}<L$.

For the front form, the situation turns out to be more complicated. Using Poisson resummation one can derive the finite-volume version of the canonical light-cone commutator, at $x^{+}=0$, which is a periodic sign function,

$$
\Delta_{F F}\left(x^{+}=0, x^{-}\right)=-\frac{1}{4} \sum_{n} \operatorname{sgn}\left(x^{-}+2 L n\right)+x^{-} / 4 L .
$$

This coincides with (174) if $x^{-}$is restricted to lie between $-L$ and $L$.

For $x^{+} \neq 0$, I have evaluated $\Delta_{F F}$ numerically. The result is shown in Fig. 5 as a function of the dimensionless variables $v \equiv x^{-} / 2 L, w=m^{2} L x^{+} / 2$. For large values of $w, \Delta_{F F}$ attains a very irregular shape, though numerically the representation (179) converges to a periodic function. The most important observation, however, is that $\Delta_{F F}$ does not vanish outside the light-cone, i.e. for $x^{-}<0$, if $x^{+}>0$ as in Fig. 5. This a clear violation of microcausality as has first been observed in (Scheu 1997).

As already stated, it is not straightforward to confirm these findings analytically. Poisson resummation does not work; first, because of the weak localization 
Fig. 5. $\Delta_{F F}(v, w)$ as a function of $v=x^{-} / 2 L . w=10000, N=70$. It does not vanish outside the LC, $-1<v<0$.

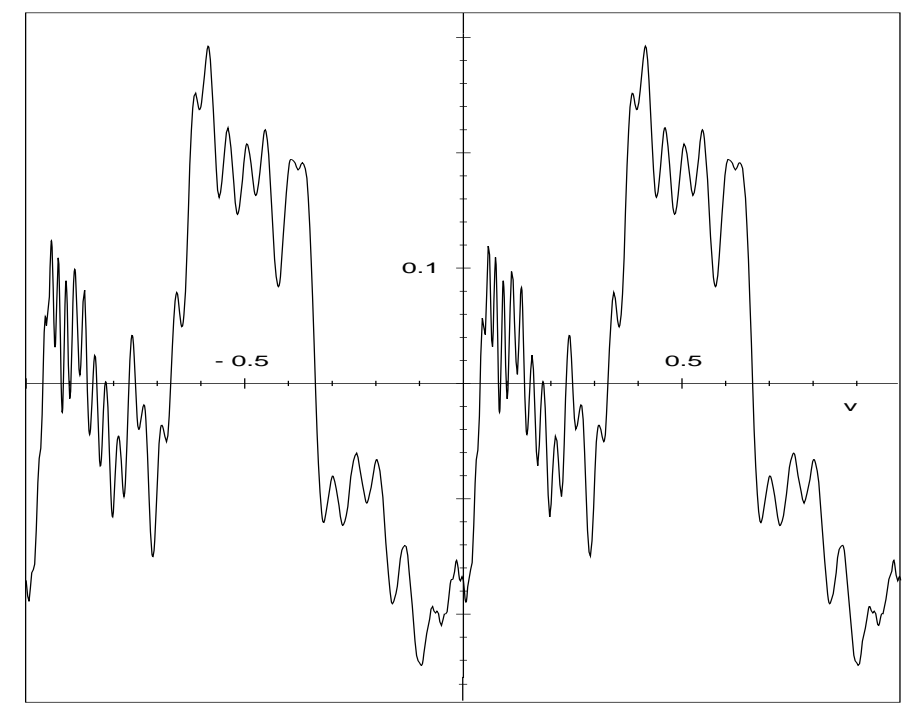

properties of $\Delta$ in $x^{-}$(asymptotically, is goes like $\left.\left(x^{-}\right)^{-1 / 4}\right)$; second, and even worse, because the zero mode $I\left(k^{+}=0\right)$ does not exist for $x^{+} \neq 0$. Nevertheless, an independent confirmation of causality violation can be obtained from resumming (179) in terms of Bernoulli polynomials, thereby replacing the Fourier series by a (rapidly converging) power series in $w$. The result is shown in Fig. 6 for $w=5$. There is nice agreement with the Fourier representation (179) (and no Gibbs phenomenon, as expected). Again, causality violation is obvious.

From a technical point of view, the violation of causality is not really astonishing. We are replacing the integral over the severely oscillating function $I\left(k^{+}\right)$ by a Riemann sum with equidistant grid points. In this way, we are sampling the integrand in such a way that the huge cancellations present in the integral do not take place. Instead, for small $k^{+}$, we replace $I\left(k^{+}\right)$by a random 'staircase' function which in the end produces the 'noise' seen in Fig. 5.

At this point a natural questions arises: is there a remedy for the causality violation? The answer is positive. We have found two ways around the problem ${ }^{6}$, both, however, with shortcomings of their own. The first way is to regularize the integral (177), replacing $\Delta$ by $\Delta_{\epsilon}$ in such a way that the associated integrand satisfies $I_{\epsilon}\left(k^{+}=0\right)=0$. One can chose e.g. a principal value regularization (Hornbostel et al. 1988) or a more sophisticated prescription (Nakanishi and Yamawaki 1977). In this way, one suppresses the oscillations and the divergence at

\footnotetext{
${ }^{6}$ For a third method, see (Chakrabarti et al. 1999).
} 
Fig. 6. Comparison of the Fourier representation (179) with the result of Bernoulli resummation (smooth, heavy line).

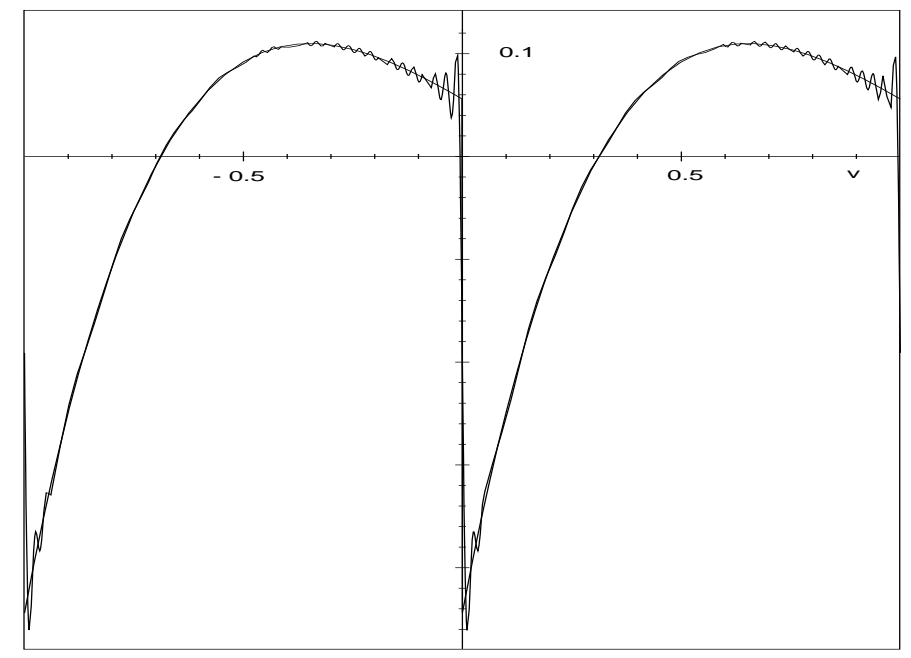

$k^{+}=0$ at the price of introducing a small causality violation of order $\epsilon$. But now $\Delta_{\epsilon}$ can be approximated by a discrete sum if the momentum grid is sufficiently fine, $\triangle k^{+} \ll \epsilon$. The order of limits, however, becomes important. First, one has to perform the infinite-volume limit, $\triangle k^{+} \rightarrow 0, L \rightarrow \infty$, and only then the limit $\epsilon \rightarrow 0$. For this method, Poisson resummation should work (Salmons et al. 1999). However, it seems somewhat awkward and not very economic to perform two regularizations (finite $L$ and $\epsilon$ ).

An alternative way of resolving the problem is the following: instead of an equally spaced grid à la DLCQ (i.e. $\triangle k_{n}^{+}=$const) one can chose an adapted momentum grid with spacing $\triangle k_{n}^{+} \sim 1 / n$ for small $n$. In this way, one is sampling the small- $k^{+}$-region of $I\left(k^{+}\right)$in a more reasonable way. Practically, the method amounts to viewing $\Delta_{I F}$ as the correct finite-volume expression and replacing $x^{0}$ and $x^{1}$ by $\left(x^{+} \pm x^{+}\right) / 2$, respectively. This is equivalent to introducing new discrete momenta, $k_{n}^{ \pm} \equiv \omega_{n} \pm k_{n}^{1}$.

As a result, the point $k^{+}=0$ becomes an accumulation point of the momentum grid which leads to a causal finite-volume representation $\Delta_{c}\left(x^{+}, x^{-}\right)$of $\Delta$ (see Fig. 7). This function, however, is no longer periodic in $x^{-}$. We thus find that, with a light-like direction being compactified, one cannot have both, periodicity and causality. On the other hand, the regularization method above seems to suggest that the causality violation is in some sense 'small' and thus may have a minor effect on the calculation of observables. Whether this statement is true has still to be worked out in detail. 
Fig. 7. The causal commutator $\Delta_{c}$ as a function of $v \cdot x^{+} / 2 L=0.2, m L=50, N=50$.

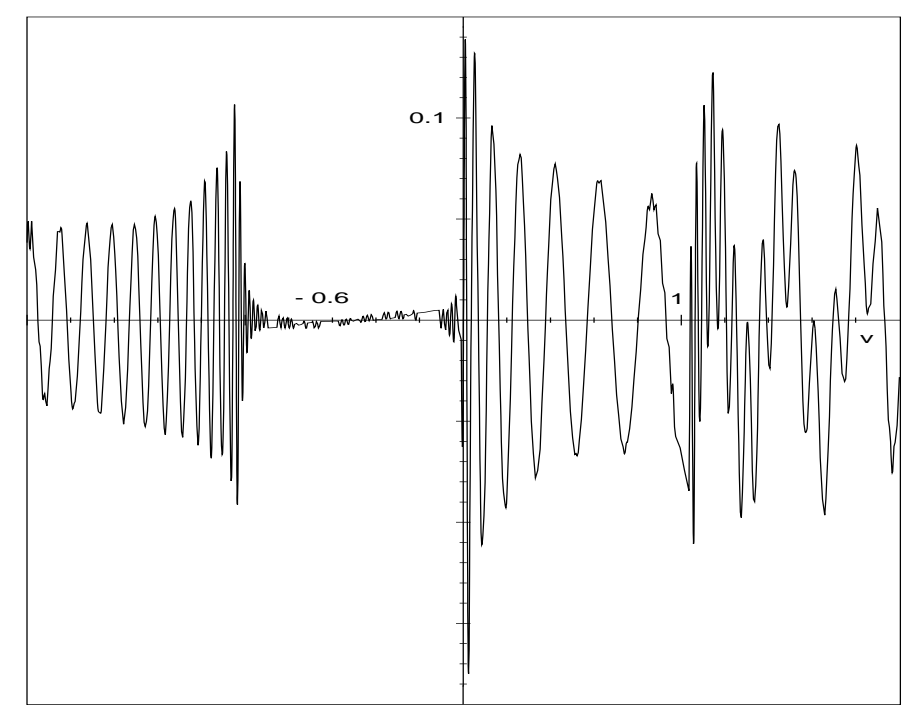

\subsection{The Functional Schrödinger Picture}

Having discussed some difficulties of light-cone quantization in a finite 'box' we go back to the infinite volume and consider yet another method of quantization, the functional Schrödinger picture. The idea of this method is to mimic quantum mechanics within quantum field theory. States are described as functionals $\Psi[\phi]$ depending on the field(s) $\phi$, while operators are combinations of multiplication by functionals of $\phi$ and functional differentiation with respect to $\phi$.

Bosons (Instant Form). For a free massive scalar field in two dimensions one finds the following results using instant form dynamics (Jackiw 1987). The canonical momentum (operator) acts via differentiation,

$$
\hat{\pi}(x) \Psi[\phi]=-i \frac{\delta}{\delta \phi(x)} \Psi[\phi] .
$$

The ground state $\Psi_{0}$ of the system, the Fock vacuum, is obtained by direct analogy with the harmonic oscillator. One rewrites the vacuum annihilation condition $a(k)|0\rangle=0$ as a functional differential equation,

$$
\left[\int d y \Omega(x-y) \phi(y)+\frac{\delta}{\delta \phi(x)}\right] \Psi_{0}[\phi]=0
$$


which is solved by a Gaussian,

$$
\Psi_{0}[\phi] \sim \exp \left[-\frac{1}{2}(\phi, \Omega \phi)\right] .
$$

Here we have introduced the 'quadratic form',

$$
(\phi, \Omega \phi) \equiv \int d x d y \phi(x) \Omega(x-y) \phi(y),
$$

using the kernel (or covariance) $\Omega(x-y$ ). The latter is defined by its Fourier transform,

$$
\Omega(k) \equiv \sqrt{k^{2}+m^{2}} \equiv \omega_{k},
$$

which is nothing but the on-shell energy of a free massive scalar. For later use, it is important to note that the instant form covariance $\Omega$ is explicitly mass dependent. As a consequence, if we have two free scalars of masses $m_{1}$ and $m_{2}$, respectively, their Fock vacua are related by a Bogolubov transformation,

$$
\left|\Omega_{2}\right\rangle=U_{21}\left|\Omega_{1}\right\rangle,
$$

with the unitary operator $U_{21}$ explicitly given by

$$
U_{21}=\exp \int \frac{d k}{4 \pi} \theta_{k}\left[a_{1}(k) a_{1}(-k)-a_{1}^{\dagger}(k) a_{1}^{\dagger}(-k)\right] .
$$

$\theta_{k}$ is the Bogolubov angle. We mention in passing that in order to properly define $U_{21}$ as an operator one should work in finite volume to avoid infrared singularities (Heinzl 1998). Otherwise the two vacuum states have vanishing overlap. Within the functional Schrödinger picture this has been analysed by (Jackiw 1987).

Fermions (Instant Form). For fermions, the situation is slightly more involved. Note that even within the instant form, the Dirac Lagrangian represents a first-order system, so that one expects some similarities with light-cone quantization in this case. This expectation will indeed turn out to be true. The instant form fermionic field operators are given by (Kiefer and Wipf 1994)

$$
\hat{\psi}_{\alpha}=\frac{1}{\sqrt{2}}\left(u_{\alpha}+\frac{\delta}{\delta u_{\alpha}^{*}}\right), \quad \hat{\psi}_{\alpha}^{\dagger}=\frac{1}{\sqrt{2}}\left(u_{\alpha}^{*}+\frac{\delta}{\delta u_{\alpha}}\right),
$$

and thus are linear combinations of multiplication by and differentiation with respect to the complex-valued Grassmann functions $u_{\alpha}(x)$ and $u_{\alpha}^{*}(x)$. These functions characterize the states, for example the ground state (Fock vacuum) which is again Gaussian,

$$
\Psi_{0}\left[u, u^{*}\right] \sim \exp \left(u^{*}, \Omega u\right) .
$$

For $2 d$ massive fermions, the covariance is found to be

$$
\Omega(k)=\frac{1}{\sqrt{k^{2}+m^{2}}}\left(k \sigma^{1}-m \sigma^{3}\right),
$$


with $\sigma^{1}$ and $\sigma^{3}$ the standard Pauli matrices. Again, $\Omega$ is explicitly mass dependent. In the massless case, $m=0$, it becomes particularly simple,

$$
\Omega(k)=\operatorname{sgn}(k) \sigma^{1},
$$

or, after Fourier transformation,

$$
\Omega(x-y)=\frac{i}{\pi} \mathcal{P} \frac{1}{x-y} \sigma^{1} .
$$

Here we have once more made use of the fact that the principle value $\mathcal{P}(1 / x)$ is the Fourier transform of the sign function.

Bosons (Front Form). As stated above, the latter case is somewhat similar to the generic situation in light-cone quantization. Let us again consider a massive free scalar field $\phi$ in $2 d$ with Fock expansion (157). We decompose it into positive and negative frequency part,

$$
\phi=\phi^{+}[a]+\phi^{-}\left[a^{*}\right] \equiv u+u^{*},
$$

where $\phi^{-}=\left(\phi^{+}\right)^{*}$ as $\phi$ is real. Quantization is performed by defining field operators such that the canonical light-cone commutator (122) is reproduced. The solution turns out to be somewhat more complicated than for instant form fields, namely

$$
\begin{aligned}
& \hat{\phi}^{+}\left(x^{-}\right)=u\left(x^{-}\right)+\frac{1}{2} \int d y^{-} i \Delta_{+}\left(x^{-}-y^{-}\right) \frac{\delta}{\delta u^{*}\left(y^{-}\right)}, \\
& \hat{\phi}^{-}\left(x^{-}\right)=u^{*}\left(x^{-}\right)+\frac{1}{2} \int d y^{-} i \Delta_{-}\left(x^{-}-y^{-}\right) \frac{\delta}{\delta u\left(y^{-}\right)} .
\end{aligned}
$$

$\Delta_{+}$and $\Delta_{-}$are distributions that sum up to $\Delta, \Delta_{+}+\Delta_{-}=\Delta$, and are explicitly given by

$$
i \Delta_{ \pm}\left(x^{-}\right)=\mp \ln \left( \pm x^{-}-i \epsilon\right)=\mp \ln \left|x^{-}\right| \pm \frac{i}{4} \theta\left(\mp x^{-}\right) .
$$

The ground state is annihilated by $\hat{\phi}^{+}, \hat{\phi}^{+} \Psi_{0}\left[u, u^{*}\right]=0$, which yields a functional differential equation, again with Gaussian solution,

$$
\Psi_{0}\left[u, u^{*}\right]=\exp \left[-\left(u^{*}, \Omega u\right)\right] .
$$

The covariance is given by

$$
\Omega\left(x^{-}\right)=2 i \partial^{+} \delta\left(x^{-}\right), \quad \Omega\left(k^{+}\right)=2 k^{+},
$$

and thus is a local expression which is a very peculiar finding. In momentum space, the ubiquitous longitudinal momentum $k^{+}$appears. One thing that is particularly obvious from (200) is the fact that the light-cone Fock vacuum is mass independent, $\Psi_{0}\left(m_{1}\right)=\Psi_{0}\left(m_{2}\right)$ which means that the analog of the Bogolubov transformation (189) is trivial, i.e. $U_{21}=\mathbb{1}$. This has been checked 
explicitly for several examples, including the Nambu-Jona-Lasinio model (Dietmaier et al. 1989) and bosons and fermions coupled to external sources (Heinzl 1998).

I believe that the locality of the covariance has far reaching consequences which, however, are still to be worked out in the present framework. Within ordinary Fock space language, some properties of the light-cone vacuum will be discussed in what follows.

\subsection{The Light-Cone Vacuum}

One of the basic axioms of quantum field theory states that the spectrum of the four-momentum operator is contained within the closure of the forward lightcone (Streater and Wightman 1963; Bogolubov et al. 1975). The four-momentum $P^{\mu}$ of any physical, that is, observable particle thus obeys

$$
P^{2} \geq 0, \quad P^{0} \geq 0,
$$

which is, of course, consistent with the mass-shell constraint, $p^{2}=m^{2}$. The tip of the cone, the point $P^{2}=P^{0}=0$, corresponds to the vacuum. From the spectrum condition (201) we infer that

$$
P_{0}^{2}-P_{3}^{2} \geq P_{\perp}^{2} \geq 0 \quad \text { or } \quad P^{0} \geq\left|P^{3}\right| .
$$

This implies for the longitudinal light-cone momentum,

$$
P^{+}=P^{0}+P^{3} \geq\left|P^{3}\right|+P^{3} \geq 0 .
$$

We thus have the important kinematical constraint that physical states must have nonnegative longitudinal momentum,

$$
\left\langle\text { phys }\left|P^{+}\right| \text {phys }\right\rangle \geq 0 \text {. }
$$

The spectrum of $P^{+}$is thus bounded from below. Due to Lorentz invariance, the vacuum $|0\rangle$ must have vanishing four-momentum, and in particular

$$
P^{+}|0\rangle=0 .
$$

Therefore, the vacuum is an eigenstate of $P^{+}$with the lowest possible eigenvalue, namely zero. We will be interested in the phenomenon of spontaneous symmetry breaking, i.e. in the question whether - roughly speaking - the vacuum is degenerate. Let us thus analyse whether there is another state, $\left|p^{+}=0\right\rangle$, having the same eigenvalue, $p^{+}=0$, as the vacuum. If so, it must be possible to create this state from the vacuum with some operator $U$,

$$
\left|p^{+}=0\right\rangle=U|0\rangle,
$$

where $U$ must not produce any longitudinal momentum. Note that within ordinary quantization such a construction is straightforward and quite common, 
for example in BCS theory. A state with vanishing three-momentum can be obtained via

$$
|\mathbf{p}=0\rangle=\int d^{3} k f(\mathbf{k}) a^{\dagger}(\mathbf{k}) a^{\dagger}(-\mathbf{k})|0\rangle,
$$

where $f$ is an arbitrary wave function. Evidently, the contributions from modes with positive and negative momenta cancel each other. It is obvious as well, that within light-cone quantization things must be different as there cannot be an analogous cancellation for the longitudinal momenta which are always nonnegative. Instead, one could imagine something like

$$
\left|p^{+}=\mathbf{p}_{\perp}=0\right\rangle=\int_{0}^{\infty} d k^{+} \int d^{2} k_{\perp} f\left(\mathbf{k}_{\perp}\right) \delta\left(k^{+}\right) a^{\dagger}\left(k^{+}, \mathbf{k}_{\perp}\right) a^{\dagger}\left(k^{+},-\mathbf{k}_{\perp}\right)|0\rangle .
$$

The problem thus boils down to the question whether there are Fock operators carrying light-cone momentum $k^{+}=0$. As we have seen in Subsection 3.4, there are no such operators, and a construction like (208) is impossible.

The only remaining possibility is that, if $U$ contains a creation operator $a^{\dagger}\left(k^{+}>0\right)$ carrying longitudinal momentum $k^{+} \neq 0$, there must be annihilators that annihilate exactly the same amount $k^{+}$of momentum. Thus, after Wick ordering, $U$ must have the general form

$$
\begin{aligned}
U=\langle 0|U| 0\rangle & +\int_{k^{+}>0} d k^{+} f_{2}\left(k^{+}\right) a^{\dagger}\left(k^{+}\right) a\left(k^{+}\right) \\
& +\int_{p^{+}>0} d p^{+} \int_{k^{+}>0} d k^{+} f_{3}\left(k^{+}, p^{+}\right) a^{\dagger}\left(p^{+}+k^{+}\right) a\left(p^{+}\right) a\left(k^{+}\right) \\
& +\int_{p^{+}>0} d p^{+} \int_{k^{+}>0} d k^{+} \tilde{f}_{3}\left(k^{+}, p^{+}\right) a^{\dagger}\left(p^{+}\right) a^{\dagger}\left(k^{+}\right) a\left(k^{+}+p^{+}\right) \\
& +\ldots
\end{aligned}
$$

It follows that the light-cone vacuum $|0\rangle$ is an eigenstate of $U$,

$$
U|0\rangle=\langle 0|U| 0\rangle|0\rangle .
$$

As we only deal with rays in Hilbert space, the action of $U$ on the vacuum does not create a state distinct from the vacuum. One says that the vacuum is trivial. Put differently, 'there is no vacuum but the Fock vacuum'. Note that this is actually consistent with our findings in the last subsection: the light-cone vacuum is the same irrespective of the masses of the particles; the Bogolubov transformation present in the instant form becomes trivial.

Let us analyse the dynamical implications of the general result (210). Any quantity that is obtained by integrating some functional of the fields over $x^{-}$, i.e.,

$$
F[\phi]=\int d x^{-} \mathcal{F}[\phi],
$$


is of the form (209), because the integration can be viewed as a projection onto the longitudinal momentum sector $k^{+}=0$. The most important examples for such quantities are the Poincaré generators, as is obvious from the representations $(107,108)$. This implies in particular that the trivial light-cone vacuum is an eigenstate of the fully interacting light-cone Hamiltonian $P^{-}$,

$$
P^{-}|0\rangle=\left\langle 0\left|P^{-}\right| 0\right\rangle|0\rangle .
$$

This can be seen alternatively by considering

$$
P^{+} P^{-}|0\rangle=P^{-} P^{+}|0\rangle=0,
$$

which says that $P^{-}|0\rangle$ is a state with $k^{+}=0$, so that $P^{-}$must have a Fock representation like $U$ in (209).

The actual value of $\left\langle 0\left|P^{-}\right| 0\right\rangle$ is not important at this point as it only defines the zero of light-cone energy. Note that, within the instant form, the Fock or trivial vacuum is not an eigenstate of the full Hamiltonian as the latter usually contains terms with only creation operators where positive and negative threemomenta compensate to zero as in (207). The instant-form vacuum thus is unstable under time evolution. Such a vacuum, a typical example of which is provided by (207), is called 'nontrivial'.

This concludes the general discussion of light-cone quantum field theory. Having understood the foundations of the approach we are now heading for the applications. We begin with a survey of the light-cone Schrödinger equation. 


\section{Light-Cone Wave Functions}

In this section we collect some basic facts about the eigenvalue problem of the light-cone Hamiltonian, or, in other words, about the light-cone Schrödinger equation and its solutions, the light-cone wave functions. Throughout this section, I will use the conventions of (Brodsky and Lepage 1989).

\subsection{Kinematics}

To set the stage for the definition of light-cone wave functions let me first introduce some relevant kinematical variables. Consider a system of many particles which, for the time being, will be assumed as noninteracting. Let the $i^{\text {th }}$ particle have mass $m_{i}$ and light-cone four-momentum

$$
p_{i}=\left(p_{i}^{+}, \mathbf{p}_{\perp i}, p_{i}^{-}\right) .
$$

As the particles are free, the total four-momentum is conserved and thus given by the sum of the individual momenta,

$$
P=\sum_{i} p_{i}
$$

The individual particles are on-shell, so their four-momentum squared is

$$
p_{i}^{2}=p_{i}^{+} p_{i}^{-}-p_{\perp i}^{2}=m_{i}^{2} .
$$

The square of the total four-momentum, on the other hand, defines the free invariant mass squared,

$$
P^{2}=P^{+} P^{-}-P_{\perp}^{2} \equiv M_{0}^{2}
$$

a quantity that will become important later on. We introduce relative momentum coordinates $x_{i}$ and $\mathbf{k}_{\perp i}$ via

$$
\begin{aligned}
p_{i}^{+} & \equiv x_{i} P^{+} \\
\mathbf{p}_{\perp i} & \equiv x_{i} \mathbf{P}_{\perp}+\mathbf{k}_{\perp i} .
\end{aligned}
$$

Thus, $x_{i}$ and $\mathbf{k}_{\perp i}$ denote the longitudinal momentum fraction and the relative transverse momentum of the $i^{t h}$ particle, respectively. Comparing with (215) we note that these variables have to obey the constraints

$$
\sum_{i} x_{i}=1, \quad \sum_{i} \mathbf{k}_{\perp i}=0 .
$$

A particularly important property of the relative momenta is their boost invariance. To show this we calculate, using (86),

$$
x_{i}^{\prime}=e^{\omega} p_{i}^{+} / e^{\omega} P^{+}=x_{i}
$$


From this and (88) we find in addition

$$
\mathbf{k}_{\perp i}^{\prime}=\mathbf{p}_{\perp i}^{\prime}-x_{i} \mathbf{P}_{\perp}^{\prime}=\mathbf{p}_{\perp i}+\mathbf{v}_{\perp} p_{i}^{+}-x_{i}\left(\mathbf{P}_{\perp}+\mathbf{v}_{\perp} P^{+}\right)=\mathbf{k}_{\perp i},
$$

which indeed proves the frame independence of $x_{i}$ and $\mathbf{k}_{\perp i}$.

Let us calculate the total light-cone energy of the system in terms of the relative coordinates. Making use of the constraints (220), we obtain

$$
\begin{aligned}
P^{-} & =\sum_{i} p_{i}^{-}=\sum_{i} \frac{p_{\perp i}^{2}+m_{i}^{2}}{p_{i}^{+}}=\sum_{i} \frac{\left(x_{i} \mathbf{P}_{\perp}+\mathbf{k}_{\perp i}\right)^{2}+m_{i}^{2}}{x_{i} P^{+}} \\
& =\frac{1}{P^{+}}\left(P_{\perp}^{2}+\sum_{i} \frac{k_{\perp i}^{2}+m_{i}^{2}}{x_{i}}\right) \equiv P_{\mathrm{CM}}^{-}+P_{\mathrm{r}}^{-} .
\end{aligned}
$$

This is another important result: the light-cone Hamiltonian $P^{-}$separates into a center-of-mass term,

$$
P_{\mathrm{CM}}^{-}=P_{\perp}^{2} / P^{+},
$$

and a term containing only the relative coordinates,

$$
P_{\mathrm{r}}^{-}=\frac{1}{P^{+}}\left(\sum_{i} \frac{k_{\perp i}^{2}+m_{i}^{2}}{x_{i}}\right)=\frac{M_{0}^{2}}{P^{+}} .
$$

The second identity, which states that $P_{r}^{-}$is essentially the free invariant mass squared, follows upon multiplying (223) by $P^{+}$,

$$
P^{+} P_{r}^{-}=P^{+} P^{-}-P_{\perp}^{2}=M_{0}^{2}=\sum_{i} \frac{k_{\perp i}^{2}+m_{i}^{2}}{x_{i}} .
$$

To simplify things even more, one often goes to the 'transverse rest frame' where $\mathbf{P}_{\perp}$ and therefore the center-of-mass Hamiltonian $P_{\mathrm{CM}}^{-}$from (224) vanish.

In the interacting case, the dynamical Poincaré generators acquire 'potential' terms as I have shown in Section 3.1. The light-cone Hamiltonian, e.g. becomes $P^{-}=P_{0}^{-}+V$, leading to a four-momentum squared

$$
P^{2}=P^{+}\left(P_{0}^{-}+V\right)-P_{\perp}^{2} \equiv M^{2} .
$$

Subtracting (217) we obtain the useful relation,

$$
M^{2}-M_{0}^{2}=P^{+} V \equiv W .
$$

In the quantum theory, this operator identity, when applied to physical states, is nothing but the light-cone Schrödinger equation.

Summarizing we note that the special behavior under boosts together with the transverse Galilei invariance leads to frame independent relative coordinates and a separation of the center-of-mass motion, reminiscent of ordinary nonrelativistic physics. This is at variance with the instant form, where the appearance of the notorious square root in the energy, $P^{0}=\left(\mathbf{P}^{2}+M_{0}^{2}\right)^{1 / 2}$, prohibits a similar separation of variables. 


\subsection{Definition of Light-Cone Wave Functions}

Let us first stick to a discrete notation and, for the time being, stay in $1+1$ dimensions. We thus have a Fock basis of states

$$
\begin{aligned}
& |n\rangle=a_{n}^{\dagger}|0\rangle, \\
& |m, n\rangle=a_{m}^{\dagger} a_{n}^{\dagger}|0\rangle, \\
& \vdots \\
& \left|n_{1}, \ldots, n_{N}\right\rangle=a_{n_{1}}^{\dagger} \ldots a_{n_{N}}^{\dagger}|0\rangle .
\end{aligned}
$$

This leads to a completeness relation defining the unit operator in Fock space,

$$
\begin{aligned}
\mathbb{1} & =|0\rangle\left\langle 0\left|+\sum_{n>0}\right| n\right\rangle\left\langle n\left|+\frac{1}{2} \sum_{m, n>0}\right| m, n\right\rangle\langle m, n|+\ldots \\
& =|0\rangle\left\langle 0\left|+\sum_{N=1}^{\infty} \frac{1}{N !} \sum_{n_{1}, \ldots, n_{N}>0}\right| n_{1}, \ldots, n_{N}\right\rangle\left\langle n_{1}, \ldots, n_{N}\right| .
\end{aligned}
$$

An arbitrary state $|\psi\rangle$ can thus be expanded as

$$
|\psi\rangle=\sum_{n>0}\langle n \mid \psi\rangle|n\rangle+\frac{1}{2} \sum_{m, n>0}\langle m, n \mid \psi\rangle|m, n\rangle+\ldots
$$

The sums are such that the longitudinal momenta in each Fock sector add up to the total longitudinal momentum of $|\psi\rangle$. Note that the vacuum state does not contribute as it is orthogonal to any particle state, $\langle 0 \mid \psi\rangle=0$. The normalization of this state is obtained as

$$
\begin{aligned}
\langle\psi \mid \psi\rangle & =\sum_{n>0}|\langle n \mid \psi\rangle|^{2}+\frac{1}{2} \sum_{m, n>0}|\langle m, n \mid \psi\rangle|^{2}+\ldots \\
& =\sum_{N=1}^{\infty} \frac{1}{N !} \sum_{n_{1}, \ldots, n_{N}>0}\left|\left\langle n_{1}, \ldots, n_{N} \mid \psi\right\rangle\right|^{2} .
\end{aligned}
$$

Let us assume that the state $|\psi\rangle$ corresponds to a bound state obeying the light-cone Schrödinger equation derived from (228),

$$
\left(M^{2}-\hat{M}_{0}^{2}\right)|\psi\rangle=\hat{W}|\psi\rangle .
$$

We want to project this equation onto the different Fock sectors. For this we need the eigenvalues of the free invariant mass squared when applied to an $N$-particle state

$$
|N\rangle \equiv\left|n_{1}, \ldots n_{N}\right\rangle .
$$

We find that $|N\rangle$ is an eigenstate of $\hat{M}_{0}^{2}$,

$$
\hat{M}_{0}^{2}|N\rangle=\sum_{i=1}^{N} \frac{m_{i}^{2}}{x_{i}}|N\rangle \equiv M_{N}^{2}|N\rangle .
$$


The light-cone Schrödinger equation thus becomes a system of coupled eigenvalue equations,

$$
\left[\begin{array}{c}
\left(M^{2}-M_{1}^{2}\right)\langle l \mid \psi\rangle \\
\left(M^{2}-M_{2}^{2}\right)\langle k l \mid \psi\rangle \\
\vdots
\end{array}\right]=\left[\begin{array}{ccc}
\langle l|W| m\rangle & \langle l|W| m n\rangle & \ldots \\
\langle k l|W| m\rangle & \langle k l|W| m n\rangle & \ldots \\
\vdots & \vdots & \ddots
\end{array}\right]\left[\begin{array}{c}
\langle l \mid \psi\rangle \\
\langle k l \mid \psi\rangle \\
\vdots
\end{array}\right] .
$$

Clearly, this represents an infinite number of equations which in general will prove impossible to solve unless the matrix is very sparse and/or the matrix elements are small. The former condition is usually fulfilled as the interaction $W$ in the light-cone Hamiltonian typically changes particle number at most by two ${ }^{7}$. Assuming the matrix elements to be small amounts to dealing with a perturbative situation. This will be true for nonrelativistic bound states of heavy constituents, but not for light hadrons which we are mainly interested in. There are, however, situations where the magnitude of the amplitudes decreases enormously with the particle number $N$, so that it is a good approximation to restrict to the lowest Fock sectors. In instant form field theory this has long been known as the Tamm-Dancoff method (Tamm 1945; Dancoff 1950).

Let us turn to the more realistic case of $3+1$ dimensions in a continuum formulation. The invariant normalization of a momentum eigenstate $\left|P^{+}, \mathbf{P}_{\perp}\right\rangle \equiv$ $|\boldsymbol{P}\rangle$ is given by

$$
\langle\boldsymbol{P} \mid \boldsymbol{K}\rangle=16 \pi^{3} P^{+} \delta^{3}(\boldsymbol{P}-\boldsymbol{K}) .
$$

We already know that the bare Fock vacuum is an eigenstate of the interacting Hamiltonian. It thus serves as an appropriate ground state on top of which we can build a reasonable Fock expansion. If we specialize immediately to the case of QCD, we are left with the Fock basis states

$$
\begin{aligned}
& |0\rangle \\
& \left|q \bar{q}: \boldsymbol{k}_{i}, \alpha_{i}\right\rangle=b^{\dagger}\left(\boldsymbol{k}_{1}, \alpha_{1}\right) d^{\dagger}\left(\boldsymbol{k}_{2}, \alpha_{2}\right)|0\rangle \\
& \left|q \bar{q} g: \boldsymbol{k}_{i}, \alpha_{i}\right\rangle=b^{\dagger}\left(\boldsymbol{k}_{1}, \alpha_{1}\right) d^{\dagger}\left(\boldsymbol{k}_{2}, \alpha_{2}\right) a^{\dagger}\left(\boldsymbol{k}_{3}, \alpha_{3}\right)|0\rangle
\end{aligned}
$$

In these expressions, $b^{\dagger}, d^{\dagger}$ and $a^{\dagger}$ create quarks $q$, antiquarks $\bar{q}$ and gluons $g$ with momenta $\boldsymbol{k}_{i}$ from the trivial vacuum $|0\rangle$. The $\alpha_{i}$ denote all other relevant quantum numbers, like helicity, polarization, flavor and color.

In a more condensed notation we can thus describe, say, a pion with momentum $\boldsymbol{P}=\left(P^{+}, \mathbf{P}_{\perp}\right)$, as

$$
|\pi(\boldsymbol{P})\rangle=\sum_{n, \lambda_{i}} \int \overline{\prod_{i}} d x_{i} \frac{d^{2} k_{\perp i}}{16 \pi^{3}} \psi_{n / \pi}\left(x_{i}, \mathbf{k}_{\perp i}, \lambda_{i}\right)\left|n: x_{i} P^{+}, x_{i} \mathbf{P}_{\perp}+\mathbf{k}_{\perp i}, \lambda_{i}\right\rangle,
$$

\footnotetext{
${ }^{7}$ Note that terms with only creation operators are forbidden by $k^{+}$-conservation. Still, in $1+1$ dimensions, things can become messy as interactions with polynomials of arbitrary powers in $\phi$ are allowed without spoiling renormalizability (Zinn-Justin 1996)
} 
where we have suppressed all discrete quantum numbers apart from the helicities $\lambda_{i}$. The integration measure takes care of the constraints (220) which the relative momenta in each Fock state (labeled by $n$ ) have to obey,

$$
\begin{aligned}
\overline{\prod_{i}} d x_{i} & \equiv \prod_{i} d x_{i} \delta\left(1-\sum_{j} x_{j}\right) \\
\overline{\prod_{i}} d^{2} k_{\perp i} & \equiv 16 \pi^{3} \prod_{i} d^{2} k_{\perp i} \delta^{2}\left(\sum_{j} \mathbf{k}_{\perp j}\right) .
\end{aligned}
$$

As a mnemonic rule, we note that any measure factor $d^{2} k_{\perp i}$ in (240) is always accompanied by $1 / 16 \pi^{3}$.

The most important quantities in (240) are the light-cone wave functions

$$
\psi_{n / \pi}\left(x_{i}, \mathbf{k}_{\perp i}, \lambda_{i}\right) \equiv\left\langle n: x_{i} P^{+}, x_{i} \mathbf{P}_{\perp}+\mathbf{k}_{\perp i}, \lambda_{i} \mid \pi(\boldsymbol{P})\right\rangle,
$$

which are the amplitudes to find $n$ constituents with relative momenta $p_{i}^{+}=$ $x_{i} P^{+}, \mathbf{p}_{\perp i}=x_{i} \mathbf{P}_{\perp}+\mathbf{k}_{\perp i}$ and helicities $\lambda_{i}$ in the pion. Due to the separation properties of the light-cone Hamiltonian the wave functions do not depend on the total momentum $\boldsymbol{P}$ of the pion. Applying (237) to the pion state (240), we obtain the normalization condition

$$
\sum_{n, \lambda_{i}} \int \overline{\prod_{i}} d x_{i} \frac{d^{2} k_{\perp i}}{16 \pi^{3}}\left|\psi_{n / \pi}\left(x_{i}, \mathbf{k}_{\perp i}, \lambda_{i}\right)\right|^{2}=1
$$

The light-cone bound-state equation for the pion is a straightforward generalization of (236),

$$
\left[\begin{array}{cc}
\left(M^{2}-M_{q \bar{q}}^{2}\right) & \langle q \bar{q} \mid \pi\rangle \\
\left(M^{2}-M_{q \bar{q} g}^{2}\right) & \langle q \bar{q} g \mid \pi\rangle \\
\vdots &
\end{array}\right]=\left[\begin{array}{ccc}
\langle q \bar{q}|W| q \bar{q}\rangle & \langle q \bar{q}|W| q \bar{q} g\rangle & \ldots \\
\langle q \bar{q} g|W| q \bar{q}\rangle & \langle q \bar{q} g|W| q \bar{q} g\rangle & \ldots \\
\vdots & \vdots & \ddots
\end{array}\right]\left[\begin{array}{c}
\langle q \bar{q} \mid \pi\rangle \\
\langle q \bar{q} g \mid \pi\rangle \\
\vdots
\end{array}\right]
$$

If a constituent picture for the pion were true, the valence state would dominate,

$$
\left|\psi_{2 / \pi}\right|^{2} \gg\left|\psi_{n / \pi}\right|^{2}, \quad n>2,
$$

and, in the extreme case, the pion wave function would be entirely given by the projection $\langle q \bar{q} \mid \pi\rangle$ onto the valence state. All the higher Fock contributions would vanish and the unitarity sum (244) would simply reduce to

$$
\sum_{\lambda \lambda^{\prime}} \int_{0}^{1} d x \int \frac{d^{2} k_{\perp}}{16 \pi^{3}}\left|\psi_{q \bar{q} / \pi}\left(x, \mathbf{k}_{\perp}, \lambda, \lambda^{\prime}\right)\right|^{2}=1 .
$$

We will later discuss a model where this is indeed a good approximation to reality. 


\subsection{Properties of Light-Cone Wave Functions}

Let us rewrite the light-cone bound-state equation (236) by collecting all lightcone wave functions $\psi_{n}=\langle n \mid \psi\rangle$ into a vector $\Psi$,

$$
\Psi=\frac{W \Psi}{M^{2}-M_{0}^{2}} .
$$

From this expression it is obvious that all light-cone wave functions tend to vanish whenever the denominator

$$
\epsilon \equiv M^{2}-M_{0}^{2}=M^{2}-\left(\sum_{i} p_{i}\right)^{2}=M^{2}-\sum_{i} \frac{k_{\perp i}^{2}+m_{i}^{2}}{x_{i}}
$$

becomes very large. This quantity measures how far off energy shell the total system, i.e. the bound state is,

$$
P^{-}-\sum_{i} p_{i}^{-}=\epsilon / P^{+}
$$

For this reason, $\epsilon$ is sometimes called the 'off-shellness' (Namysłowski 1985; Lavelle et al. 1987). We thus learn from (248) that there is only a small overlap of the bound state with Fock states that are far off shell. This implies the limiting behavior

$$
\psi\left(x_{i}, \mathbf{k}_{\perp i}, \lambda_{i}\right) \rightarrow 0 \quad \text { for } x_{i} \rightarrow 0, k_{\perp i}^{2} \rightarrow \infty .
$$

These boundary conditions are related to the self-adjointness of the light-cone Hamiltonian and to the finiteness of its matrix elements. Analogous criteria have been used recently to relate wave functions of different Fock states $n$ (Antonuccio et al. 1997) and to analyse the divergence structure of light-cone perturbation theory (Burkardt 1998).

Omitting spin, flavor and color degrees of freedom, a light-cone wave function will be a scalar function $\phi\left(x_{i}, \mathbf{k}_{\perp i}\right)$ of the parameter $\epsilon$. This is used for building models, the most common one being to assume a Gaussian behavior, originally suggested by (Terent'ev 1976),

$$
\phi\left(x_{i}, \mathbf{k}_{\perp i}\right)=N \exp \left(-|\epsilon| / \beta^{2}\right),
$$

where $\beta$ measures the size of the wave function in momentum space. Note, however, that a Gaussian ansatz is in conflict with perturbation theory which is the appropriate tool to study the high $-\mathbf{k}_{\perp}$ behavior and indicates a power decay of the renormalized wave function - up to possible logarithms (Brodsky and Lepage 1989). For the unrenormalized wave functions the boundary conditions (251) are violated unless one uses a cutoff as a regulator (see next section).

As the off-shellness $\epsilon$ is the most important quantity characterizing a lightcone wave function let us have a closer look by specializing to the simplest possible system, namely two bound particles of equal mass. One can think of 
this, for instance, as the valence wave function of the pion or positronium. The off-shellness becomes

$$
\epsilon=M^{2}-\frac{k_{\perp}^{2}+m^{2}}{x(1-x)}=-\frac{1}{x(1-x)}[M^{2}\left(x-\frac{1}{2}\right)^{2}+\underbrace{\frac{4 m^{2}-M^{2}}{4}}_{\geq 0}+k_{\perp}^{2}] .
$$

The second term in square brackets is positive because, for a bound state, the binding energy,

$$
E=M-2 m
$$

is negative so that $2 m>M$. As a result, the off-shellness is always negative. Only for free particles it is zero, because all momentum components (including the energy) sum up to the total momentum. In this case, each individual term in (253) vanishes,

$$
M=2 m, \quad x=\frac{1}{2}, \quad \mathbf{k}_{\perp}=0 .
$$

It follows that the light-cone wave function of a two-particle system (composed of equal-mass constituents) with the binding energy switched off 'adiabatically', is of the form

$$
\phi\left(x, \mathbf{k}_{\perp}\right) \sim \delta(x-1 / 2) \delta^{2}\left(\mathbf{k}_{\perp}\right)
$$

\subsection{Examples of Light-Cone Wave Functions}

From the discussion above, one expects that for weak binding, in particular for nonrelativistic systems, the wave functions will be highly peaked around $x=1 / 2$ (in the equal mass case) and $\mathbf{k}_{\perp}=0$. Let us check this explicitly for hydrogenlike systems which constitute our first example (Lepage et al. 1981).

Example 1: Hydrogen-like Systems. Let me recall the ordinary Schrödinger equation of the Coulomb problem written in momentum space,

$$
\left(E-\frac{\mathbf{p}^{2}}{2 m}\right) \psi(\mathbf{p})=\int \frac{d^{3} k}{(2 \pi)^{3}} V(\mathbf{p}-\mathbf{k}) \psi(\mathbf{k})=\int \frac{d^{3} k}{(2 \pi)^{3}} \frac{4 \pi \alpha}{(\mathbf{p}-\mathbf{k})^{2}} \psi(\mathbf{k}) .
$$

This integral equation looks very similar to a light-cone Schrödinger equation within a two-particle truncation. The Coulomb kernel is due to the exchange of an instantaneous photon having a propagator proportional to $\delta\left(x^{0}\right)$. One can actually solve the Coulomb problem directly in momentum space (Fock 1935; Bethe and Salpeter 1957) but for our purposes it is simpler just to Fourier transform the ground state wave function $\psi_{0}(r)=N \exp (-m \alpha r)$, yielding

$$
\psi_{0}(\mathbf{p})=8 \pi N \frac{m \alpha}{\left(\mathbf{p}^{2}+m^{2} \alpha^{2}\right)^{2}}
$$

with $\alpha=e^{2} / 4 \pi=1 / 137$ being the fine structure constant, $m$ the reduced mass and $\mathbf{p}$ the relative momentum. 
How does this translate into the light-cone language? To answer this question, we go to the particle rest frame with $\mathbf{P}=0$ or $P^{+}=P^{-}=M$ and $\mathbf{P}_{\perp}=0$, implying $\mathbf{p}_{\perp i}=\mathbf{k}_{\perp i}$. In this frame, the nonrelativistic limit is defined by the following inequalities for the constituent masses and momenta (in ordinary instant-form coordinates),

$$
p_{i}^{0}-m_{i} \simeq \frac{\mathbf{p}_{i}^{2}}{2 m_{i}} \ll\left|\mathbf{p}_{i}\right| \ll m_{i} .
$$

The prototype systems in this class are of hydrogen type where we have for binding energy and r.m.s. momentum,

$$
\begin{aligned}
& |E|=\frac{\left\langle\mathbf{p}^{2}\right\rangle}{2 m}=\frac{m \alpha^{2}}{2}, \\
& \langle p\rangle=m \alpha
\end{aligned}
$$

In this case, the hierarchy (259) becomes

$$
\frac{\alpha^{2}}{2} \ll \alpha \ll 1,
$$

which is fulfilled to a very good extent in view of the smallness of $\alpha$.

Consider now the longitudinal momentum of the $i^{\text {th }}$ constituent,

$$
p_{i}^{+}=p_{i}^{0}+p_{i}^{3} \simeq m_{i}+\frac{\mathbf{p}_{i}^{2}}{2 m_{i}}+p_{i}^{3}=x_{i} P^{+}=x_{i} M .
$$

We thus find that we should replace $p_{i}^{3}$ in instant-form nonrelativistic wave functions by

$$
p_{i}^{3}=x_{i} M-m_{i},
$$

where we neglect terms of order $\mathbf{p}_{i}^{2} / m_{i}$. Let us analyze the consequences for the off-shellness. The latter is in ordinary coordinates

$$
\epsilon=M^{2}-M_{0}^{2}=\left(M+\sum_{i} p_{i}^{0}\right)\left(M-\sum_{i} p_{i}^{0}\right) .
$$

We thus need

$$
\sum_{i} p_{i}^{0} \simeq \sum_{i} m_{i}+\sum_{i} \frac{\mathbf{p}_{i}^{2}}{2 m_{i}}=M-E+\sum_{i} \frac{\mathbf{p}_{i}^{2}}{2 m_{i}},
$$

with $E=M-\sum m_{i}$ denoting the mass-defect, which is a small quantity, $E \ll$ $M$. The off-shellness (265), therefore, becomes

$$
\epsilon \simeq 2 M\left(E-\sum_{i} \mathbf{p}_{i}^{2} / 2 m_{i}\right) \simeq-2 M \sum_{i} \frac{k_{\perp i}^{2}+\left(M x_{i}-m_{i}\right)^{2}}{2 m_{i}}
$$


where we have performed the replacement (264) in the second identity. The light-cone wave functions will be peaked where the off-shellness is small, that is, for

$$
x_{i}=m_{i} / M, \quad \text { and } \quad \mathbf{k}_{\perp \mathrm{i}}=0,
$$

as expected from the noninteracting case.

To be explicit, we consider the ground state wave function of positronium, given by (258) with the reduced mass $m$ being half the electron mass $m_{e}$. Using the replacement prescription (264) once more, we obtain

$$
\psi\left(x, \mathbf{k}_{\perp}\right)=8 \pi N \frac{m \alpha}{\left[k_{\perp}^{2}+\left(x M-m_{e}\right)^{2}+m^{2} \alpha^{2}\right]^{2}},
$$

where $M \simeq 2 m_{e}$ is the bound state mass. This result is valid for small momenta, i.e. when $k_{\perp}^{2},\left(x M-m_{e}\right)^{2} \ll m_{e}^{2}$. It is obvious from (269) that the positronium wave function is sharply peaked around $x=m_{e} / M \simeq 1 / 2$ and $k_{\perp}^{2}=0$.

Example 2: 't Hooft Model. The 't Hooft model ('t Hooft 1974; 't Hooft $1975)$ is QCD in two space-time dimensions with the number $N_{C}$ of colors being infinite. The Lagrangian is

$$
\mathcal{L}=\bar{\psi}(i \not \partial-m) \psi-\frac{1}{4} F_{\mu \nu} F^{\mu \nu}
$$

The limit of large $N_{C}$ is taken in such a way that the expression $g^{2} N_{C}, g$ denoting the Yang-Mills coupling, stays finite. In two dimensions, $g$ has mass dimension one, which renders the theory superrenormalizable and provides a basic unit of mass, namely,

$$
\mu_{0} \equiv \sqrt{g^{2} N_{C} / 2 \pi} .
$$

The model is interesting because it contains physics analogous or similar to what one finds in 'real' $\mathrm{QCD}^{8}$. First of all, the model is (trivially) confining due to the linear rise of the Coulomb potential in $2 d$. This is most easily exhibited by working in light-cone gauge, $A^{+}=0$, and eliminating $A^{-}$via Gauss's law. In this way it becomes manifest that there are no dynamical gluons in $2 d$. Within covariant perturbation theory, the Coulomb potential can be understood as resulting from the exchange of an instantaneous gluon.

In the next section, we will discuss the spontaneous breakdown of chiral symmetry in QCD. It turns out that in the ' $t$ Hooft model a similar phenomenon occurs: chiral symmetry is 'almost' spontaneously broken (Witten 1978; Zhitnitsky 1986). As a consequence, there arises a massless bound state in the chiral limit of vanishing quark mass ('t Hooft 1974; 't Hooft 1975) which we will call

\footnotetext{
${ }^{8}$ For a recent review on the 't Hooft model, see (Abdalla and Abdalla 1996).
} 
the 'pion' for brevity ${ }^{9}$. Furthermore, there is a nonvanishing quark condensate in the model which has first been calculated by (Zhitnitsky 1986),

$$
\langle 0|\bar{\psi} \psi| 0\rangle / N_{C}=-0.28868 .
$$

Note that the condensate is proportional to $N_{C}$ as it involves a color trace.

It turns out that the " $t$ Hooft model has one big advantage for the application of light-cone techniques which is due to the large $-N_{C}$ limit. The matrix elements entering the light-cone Schrödinger equation in the two-particle sector have the following $N_{C}$-dependence,

$$
\langle 2|W| 2 n\rangle \sim\left(\frac{g^{2} N_{C}}{N_{C}}\right)^{n} \sim N_{C}^{-n} .
$$

As a result, those diagrams which correspond to a change in particle number (like $2 \rightarrow 4,2 \rightarrow 6, \ldots$ ) are suppressed by powers of $1 / N_{C}$. The truncation to the two-particle sector therefore becomes exact: the 'pion' is a pure quark-antiquark state; there are no admixtures of higher Fock states. A constituent picture is thus realized, and we are left with the light-cone Schrödinger equation,

$$
\left[M^{2}-\frac{m^{2}}{x(1-x)}\right] \phi(x)=\mathcal{P} \int_{0}^{1} d y \frac{\phi(x)-\phi(y)}{(x-y)^{2}} .
$$

This expression defines the 'Coulomb problem' of the 't Hooft model. It corresponds to the first line of (245) where, as a result of the truncation, only the matrix element $W_{2} \equiv\langle q \bar{q}|W| q \bar{q}\rangle$ has been retained. We will refer to (274) as the 't Hooft equation in what follows. $\phi(x)$ denotes the valence part of the 'pion' wave function, $x$ and $y$ are the momentum fractions of the two quarks (with equal mass $m$ ) in the meson. The symbol $\mathcal{P}$ indicates that the integral is defined as a principal value ('t Hooft 1974; Gelfand and Shilov 1964). It regularizes ${ }^{10}$ the Coulomb singularity $1 /(x-y)^{2}$ in the matrix element $W_{2}$. All masses are expressed as multiples of the basic scale $\mu_{0}$ defined in (271). The eigenvalue $M$ denotes the mass of the lowest lying bound state (the 'pion'). Our objective is to calculate $M$ and $\phi$.

In his original work on the subject, ('t Hooft 1974; 't Hooft 1975), 't Hooft used the following ansatz for the wave function

$$
\phi(x)=x^{\beta}(1-x)^{\beta} .
$$

This ansatz is symmetric in $x \leftrightarrow 1-x$ (charge conjugation odd), and $\beta$ is supposed to lie between zero and one so that the endpoint behavior is nonanalytic.

\footnotetext{
${ }^{9}$ As explained in (Witten 1978; Zhitnitsky 1986), this is not in contradiction with Coleman's theorem (Coleman 1973) as the 'pion' is not a Goldstone boson.

10 (Wu 1977) has suggested a theoretical alternative to the principal value which nowadays is called 'Leibbrandt-Mandelstam prescription (Mandelstam 1983; Leibbrandt 1984). It leads to completely different physics. This apparent contradiction has only recently been clarified (Bassetto et al. 2000).
} 
As a nontrivial boundary condition, one has the exact solution of the massless case, $m=0$ (the 'chiral limit'),

$$
M^{2}=0, \quad \text { and } \phi(x)=1, \text { i.e. } \beta=0 .
$$

In this limit, the 'pion' wave function is constant, i.e. the pion has no structure and is point-like. The main effect of a nonzero quark mass is the vanishing of the wave functions at the endpoints implying a nonzero $\beta$. This suggests the following series expansion for $\beta$,

$$
\beta(m)=\beta_{1} m+\beta_{2} m^{2}+\beta_{3} m^{3}+O\left(m^{4}\right),
$$

and for the 'pion' mass squared,

$$
M^{2}=M_{1} m+M_{2} m^{2}+M_{3} m^{3}+O\left(m^{4}\right) .
$$

As is obvious from the last two expressions, we are working in the limit of small quark mass $m$. The expansion (277) shows that also $\beta$ is small in this case so that the wave function will be rather flat (for intermediate values of $x$ ). On the other hand we know from Example 1 that light-cone wave functions are highly peaked near $x=1 / 2$ in case the binding is weak. This suggests that, for small quark mass $m$, the 'pion' is rather strongly bound.

The exponent $\beta$ in (275) can actually be determined exactly by studying the small- $x$ behavior of the bound state equation (274). To this end we evaluate the principal value integral for $x \rightarrow 0$ and plug it into (274). This yields the transcendental equation ('t Hooft 1974),

$$
m^{2}-1+\pi \beta \cot \pi \beta=0 .
$$

Using this expression we can determine $\beta$ either numerically for arbitrary $m$ or analytically for small $m$, which yields the coefficients of (277),

$$
\beta=\frac{\sqrt{3}}{\pi} m+O\left(m^{3}\right) .
$$

The 'pion' mass is determined by calculating the expectation value of the lightcone Hamiltonian (274) in the state given by 't Hooft's ansatz $(275)^{11}$. This yields $M^{2}$ as a function of $\beta$ and $m$,

$$
M^{2}=\frac{2}{\beta_{1}} m+O\left(m^{2}\right) .
$$

Upon comparing with (277) and (280) the lowest order coefficient $M_{1}$, which is the slope of $M^{2}(m)$ at $m=0$, is found to be

$$
M_{1}=2 \pi / \sqrt{3} .
$$

Note that $M^{2}$ indeed vanishes in the chiral limit.

\footnotetext{
11 The relevant integrals can be found in (Bardeen et al. 1980) and (Harada et al. 1994).
} 
Having obtained an approximate solution for the mass and wave function of the 'pion' we are in the position to calculate 'observables'. It turns out that, for small $m$, all of them can be expressed in terms of the lowest order coefficient, $M_{1}$. We begin with the 'pion decay constant' (Callan et al. 1976; Zhitnitsky 1986), which is given by the 'wave function at the origin', i.e. the integral over the (momentum space) wave function

$$
f_{\pi} \equiv\left\langle 0\left|\bar{\psi} i \gamma_{5} \psi\right| \pi\right\rangle=\sqrt{\frac{N_{C}}{\pi}} \frac{M^{2}}{2 m} \int d x \phi(x)=\sqrt{\frac{N_{C}}{4 \pi}} M_{1} .
$$

The quark condensate is obtained via a sum rule using the chiral Ward identity (Zhitnitsky 1986; Heinzl 1996b),

$$
\langle 0|\bar{\psi} \psi| 0\rangle=-m \frac{f_{\pi}^{2}}{M^{2}}=-\frac{N_{C}}{4 \pi} M_{1} .
$$

Inserting the value (282) for $M_{1}$ this coincides with (272). The last identity can actually be viewed as the 'Gell-Mann-Oakes-Renner relation' (Gell-Mann et al. 1968) of the 't Hooft model,

$$
M^{2}=-4 \pi m\langle 0|\bar{\psi} \psi| 0\rangle / N_{C}+O\left(m^{2}\right),
$$

which provides a relation between the particle spectrum (the 'pion' mass) and a ground state property (the condensate). This is conceptually important because it implies that we can circumvent the explicit construction of a nontrivial vacuum state by calculating the spectrum of excited states, i.e. by solving the lightcone Schrödinger equation. The eigenvalues and wave functions actually contain information about the structure of the vacuum! This point of view has been adopted long ago in the context of chiral symmetry breaking within the (lightcone) parton model (Casher et al. 1971): "In this framework the spontaneous symmetry breakdown must be attributed to the properties of the hadron's wave function and not to the vacuum" (Casher and Susskind 1974). Related ideas have been put forward more recently in (Lenz et al. 1991).

In the above, we have been using the value for $\beta$ given in (280). One can equally well use $\beta$ as a variational parameter and minimize the expectation value of the mass operator with respect to it. This yields the same result for $M_{1}$, namely (282). The variational method, however, is better suited if one wants to go beyond the leading order in expansion (278). This has been done in (Harada et al. 1998). The results are shown in Table 2 where we list the expansion coefficients $M_{i}$ of the 'pion' mass squared, $M^{2}$, as they change with increasing number of variational parameters, $(a, b, c, d)$.

Interestingly, the value of $M_{1}$ does not change at all by enlarging the space of trial functions. $M_{2}$ and $M_{3}$, on the other hand, do change and show rather good convergence. For $M_{2}$ we finally have a relative accuracy of $8 \cdot 10^{-7}$, and for $M_{3}$ of $4 \cdot 10^{-5}$. Furthermore, the coefficients are getting smaller if one adds more basis functions, in accordance with the variational principle. The associated light-cone wave functions are shown in Fig. 8. There are only minor changes upon including 
more variational parameters. In Fig. 9 we display the eight lowest excited states. They habe been obtained using the position of the nodes as additional variational parameters (Stern 1999).

Table 2. Expansion coefficients of $M^{2}$ for the 't Hooft model obtained by successively enlarging the space of variational parameters. $M_{1}$ is the standard 't Hooft result (282). Note the good convergence towards the bottom of the table.

\begin{tabular}{lccc}
\hline \hline Ansatz & $M_{1}=2 \pi / \sqrt{3}$ & $M_{2}$ & $M_{3}$ \\
\hline 't Hooft & 3.62759873 & 3.61542218 & 0.043597197 \\
$a$ & 3.62759873 & 3.58136872 & 0.061736701 \\
$b$ & 3.62759873 & 3.58107780 & 0.061805257 \\
$c$ & 3.62759873 & 3.58105821 & 0.061795547 \\
$d$ & 3.62759873 & 3.58105532 & 0.061793082 \\
\hline \hline
\end{tabular}

Fig. 8. The light-cone wave function of the 't Hooft model 'pion' for $m=0.1$. The solid curve represents the result from 't Hooft's original ansatz, the dashed curve our best result (with maximum number of variational parameters). At the given resolution, however, the curves of all extensions of 't Hooft's ansatz $(a, b, c, d)$ lie on top of each other.

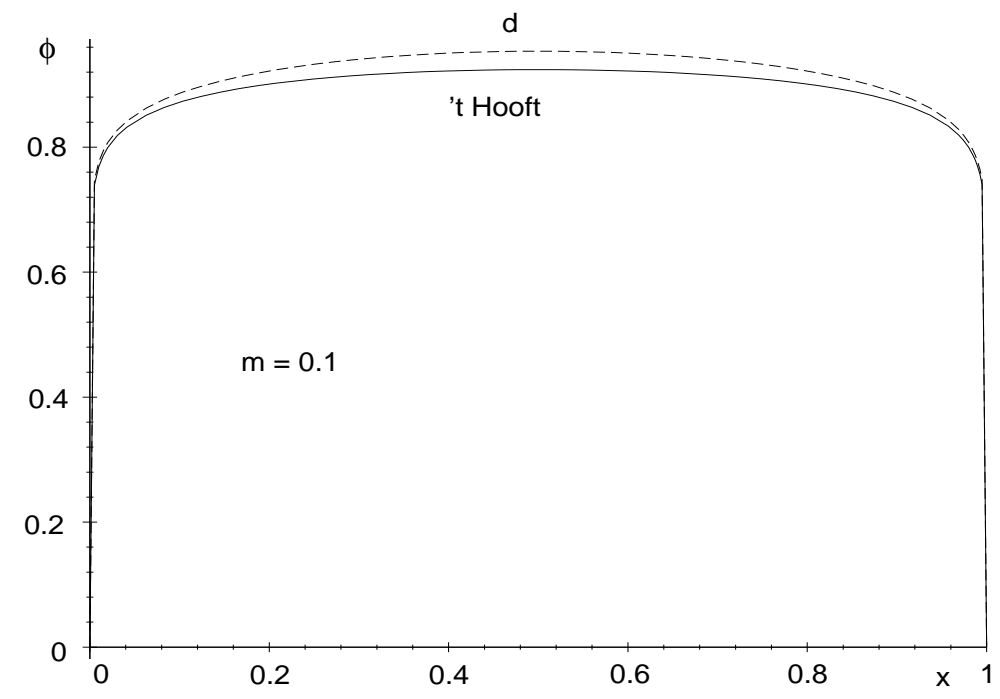


Fig. 9. Wave functions of the first eight excited states in the 't Hooft model as obtained via variational methods (Stern 1999). Note that all wave functions vanish at the end points, $x=0$ and $x=1$.

(a) -1

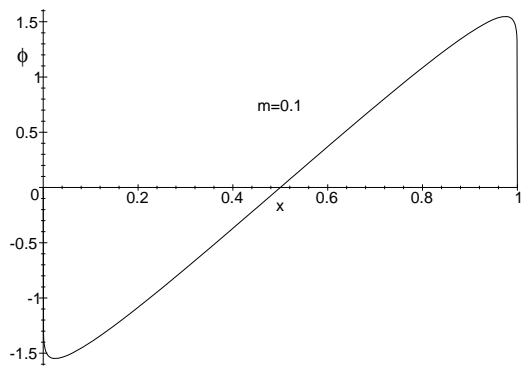

(c)

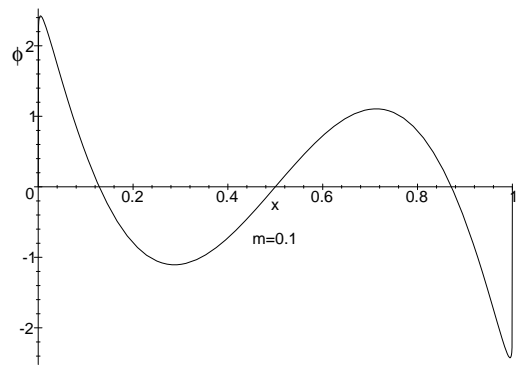

(e)

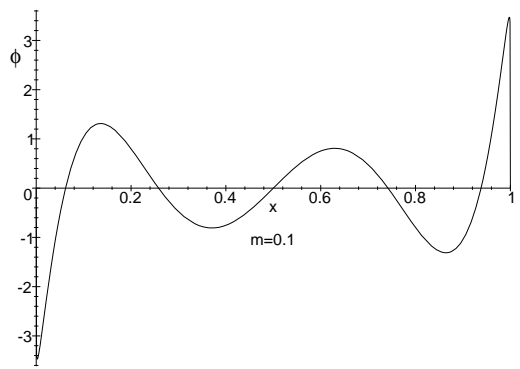

(g)

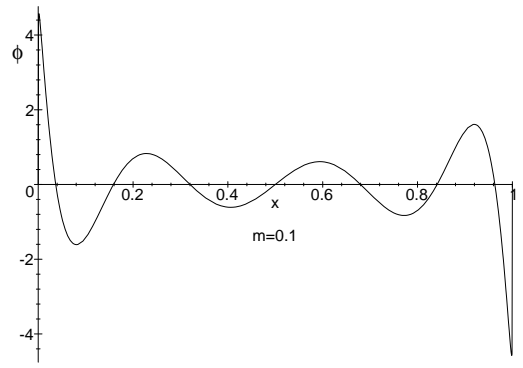

(b)

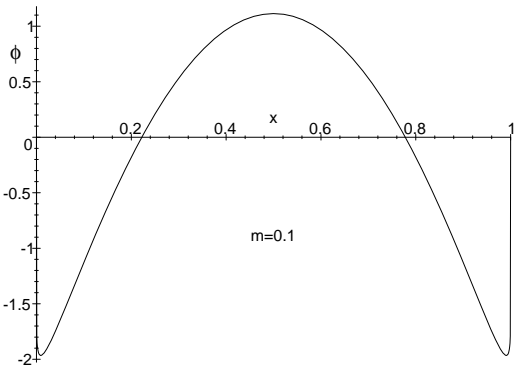

(d)

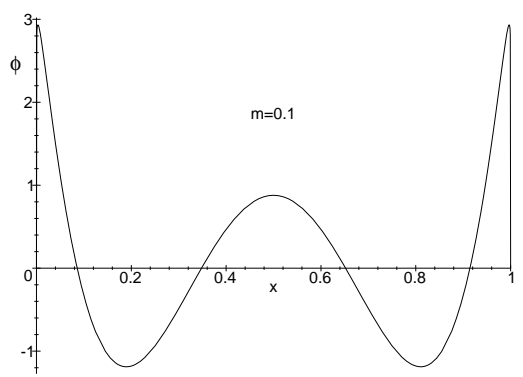

(f)

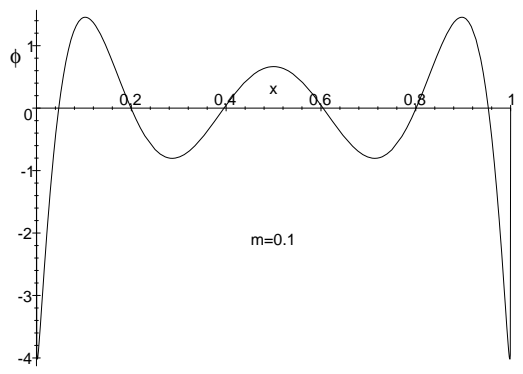

(h)

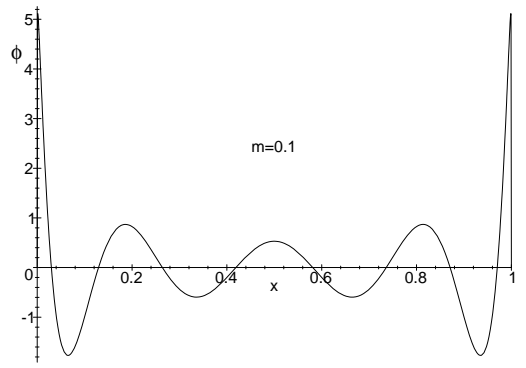


Example 3: Gaussian Model. A very simple and intuitive example of a lightcone wave function is provided by the Gaussian model in the form presented by (Lepage et al. 1981),

$$
\phi\left(x, \mathbf{k}_{\perp}\right)=N \exp \left[-\frac{1}{\Lambda^{2}} \frac{k_{\perp}^{2}+m^{2}}{x(1-x)}\right] .
$$

This is a scalar two-particle wave function that drops exponentially with the free invariant mass squared of two constituents with equal mass $m$. The shortcoming of the model is that the wave function is not derived dynamically, i.e. as a solution of a light-cone Schrödinger equation. However, it satisfies all the requirements derived in Subsection 4.3. The benefit of the model is its simplicity. The latter is even enhanced if one neglects the constituent mass $m$ by assuming $m \ll \Lambda$. Then one is left with only two parameters, the normalization $N$ and the transverse size $\Lambda$.

One enforces a constituent picture by normalizing to unity,

$$
\left\|\phi\left(x, \mathbf{k}_{\perp}\right)\right\|^{2} \equiv \int_{0}^{1} d x \int \frac{d^{2} k_{\perp}}{16 \pi^{3}} \phi^{2}\left(x, \mathbf{k}_{\perp}\right)=\frac{N^{2} \Lambda^{2}}{192 \pi^{2}} \stackrel{!}{=} 1 .
$$

This obviously relates $N$ and $\Lambda$. If we now calculate the r.m.s. transverse momentum in the bound state described by (286), we find using (287),

$$
\left\langle k_{\perp}^{2}\right\rangle \equiv \int_{0}^{1} d x \int \frac{d^{2} k_{\perp}}{16 \pi^{3}} k_{\perp}^{2} \phi^{2}\left(x, \mathbf{k}_{\perp}\right)=\frac{N^{2} \Lambda^{4}}{1920 \pi^{2}}=\Lambda^{2} / 10 .
$$

If we view the Gaussian wave function as a crude model for, say, the pion we can actually estimate the width parameter $\Lambda$. The pion is highly relativistic, so we expect its r.m.s. transverse momentum to be of the order of the constituent quark mass, $\left\langle k_{\perp}^{2}\right\rangle^{1 / 2} \simeq m_{Q} \simeq 330 \mathrm{MeV}$. This leads to a typical width of $\Lambda \simeq 1$ $\mathrm{GeV}$.

Having thus determined the two parameters of the model we could go on and calculate observables (Lepage et al. 1981). This will actually be done in the next section using a more realistic model for the (determination of) the pion wave function.

\section{The Pion Wave Function in the NJL Model}

The ultimate goal of light-cone field quantization is to derive and solve lightcone Schrödinger equations, in particular the one of QCD. This would yield hadron masses and light-cone wave functions and thus detailed information on the internal structure of mesons and baryons. In order to successfully pursue this program, a number of problems has to be overcome.

In dealing with gauge theories in a Hamiltonian framework one has to solve Gauss's law together with the light-cone specific constraints. The only known solution so far is in the light-cone gauge $A^{+}=0$ (Tomboulis 1973; Casher 1976; 
Lepage et al. 1981) which, however, is beset by infrared problems of its own - see e.g. (Bassetto et al. 1991)12. A Hamiltonian formulation analogous to the Weyl gauge, where one fixes the residual gauge freedom and solves Gauss's law after quantization, seems particularly difficult (Heinzl 1996a).

Even after successful light-cone quantization of QCD one encounters a severe problem: the theory has to be renormalized. Otherwise, light-cone wave function will not be normalizable and physically meaningful. Renormalization in a Hamiltonian framework presents enormous difficulties as there is no explicit covariance. In addition, rotational invariance is not manifest within the front form (see Section 2). Due to the lack of these important symmetries, there is an abundance of possible counterterms which even can be nonlocal, e.g. behave like $\sim 1 / k^{+}$. As a result, to the best of my knowledge, the renormalization program has not been extended beyond one loop - with one notable exception in QED (Brodsky et al. 1973).

The issue of renormalization is, of course, delicately intertwined with solving the light-cone bound state equation. The latter attempt will in general only be feasible if a Tamm-Dancoff truncation in particle number is performed (Harindranath et al. 1990). This again violates important symmetries (even gauge symmetry). One hopes, however, that a Wilsonian renormalization group explicitly taylored for this case will restore these symmetries (Wilson et al. 1994). The present status of this program is nicely reviewed in (Perry 1999).

A conceptual problem has already been mentioned. The light-cone vacuum is trivial as argued in Section 3. On the other hand, it is well known that the instant form vacuum is populated by all sorts of nonperturbative quantum fluctuations leading to nonvanishing vacuum expectation values or condensates. The whole business of QCD sum rules (Shifman et al. 1979) is based upon this picture. How do we reconcile this with the triviality of the light-cone vacuum? A possible resolution to this 'triviality problem' has been given in the last section. The spectrum of excited states actually carries implicit information on the structure of the vacuum. The task then is to make this information explicit.

The list of problems just given is yet another manifestation of the 'principle of conservation of difficulties'. In a first attempt to tackle these problems I will simply side-step most of them by considering an (instructive) model instead of QCD. This model, however, is designed to capture some important physical features of 'real' QCD. The idea is originally due to Nambu and Jona-Lasinio (NJL), who, back in 1961, invented a "Dynamical Model of Elementary Particles Based on an Analogy with Superconductivity" (Nambu and Jona-Lasinio 1961). It was meant to provide a microscopic mechanism for the generation of nucleon masses, with the mass gap being the analog of the BCS energy gap in a superconductor. Nowadays, with the nucleons replaced by quarks, the model serves as a low-energy effective theory of QCD explaining the spontaneous breakdown of chiral symmetry. Let me thus give a brief introduction to the latter phenomenon

\footnotetext{
${ }^{12}$ Recently, some progress has also been made in the covariant gauge (Srivastava and Brodsky 2000).
} 
before I come to the detailed explanation of the model.

\subsection{A Primer on Spontaneous Chiral Symmetry Breaking}

If we have a look at Table 3, which provides a list of all quark flavors, we realize that there are large differences in the quark masses as they appear in the QCD Hamiltonian. In particular, there is a hierarchy,

$$
\underbrace{m_{u}, m_{d} \ll m_{s}}_{\text {light quarks }} \ll \underbrace{m_{c}, m_{b}, m_{t}}_{\text {heavy quarks }} \text {. }
$$

As the masses of heavy and light quarks are separated by the very same scale $(\simeq 1 \mathrm{GeV})$ as the perturbative and nonperturbative regime, one expects different physics associated with those two kinds of quarks. This expectation turns out to be true. The physics of heavy quarks is governed by a symmetry called 'heavy quark symmetry' leading to a very successful 'heavy quark effective theory' (Neubert 1994). The physics of light quarks, on the other hand, is governed by chiral symmetry which we are now going to explain.

Table 3. The presently observed quark flavors. $Q / e$ is the electric charge in units of the electron charge. The (scale dependent!) quark masses are given for a scale of 1 $\mathrm{GeV}$.

\begin{tabular}{llcrl}
\hline \hline flavor & & $Q / e$ & mass & \\
\hline down & $d$ & $-1 / 3$ & 10 & $\mathrm{MeV}$ \\
up & $u$ & $+2 / 3$ & 5 & $\mathrm{MeV}$ \\
strange & $s$ & $-1 / 3$ & 150 & $\mathrm{MeV}$ \\
\hline charm & $c$ & $+2 / 3$ & 1.5 & $\mathrm{GeV}$ \\
bottom & $b$ & $-1 / 3$ & 5.1 & $\mathrm{GeV}$ \\
top & $t$ & $+2 / 3$ & 180 & $\mathrm{GeV}$ \\
\hline \hline
\end{tabular}

Let us write the QCD Hamiltonian in the following way,

$$
H_{\mathrm{QCD}}=H_{\chi}+\bar{\psi} \mathcal{M} \psi
$$

$\mathcal{M}=\operatorname{diag}\left(m_{u}, m_{d}, m_{s}\right)$ being the mass matrix for the light flavors. To a good approximation, one can set $\mathcal{M}=0$. In this case, the QCD Hamiltonian $H_{\chi}$ is invariant under the symmetry group $S U(3)_{R} \otimes S U(3)_{L}$, the chiral flavor group. Under the action of this group, the left and right handed quarks independently undergo a chiral rotation. Due to Noether's theorem, there are sixteen conserved 
quantities, eight vector charges and, more important for us, eight pseudo-scalars, the chiral charges $Q_{5}^{a}$ satisfying

$$
\left[Q_{5}^{a}, H_{\chi}\right]=0 .
$$

This states both that the chiral charges are conserved, and that $H_{\chi}$ is chirally invariant. Under parity, $Q_{5}^{a} \rightarrow-Q_{5}^{a}$. Now, if $|A\rangle$ is an eigenstate of $H_{\chi}$, so is $Q_{5}^{a}|A\rangle$ with the same eigenvalue. Thus, one expects (nearly) degenerate parity doublets in nature, which, however, do not exist empirically. The only explanation for this phenomenon is that chiral symmetry is spontaneously broken. In contradistinction to the Hamiltonian, the QCD ground state (the vacuum) is not chirally invariant,

$$
Q_{5}^{a}|0\rangle \neq 0 \text {. }
$$

For this reason, there must exist a nonvanishing vacuum expectation value, the quark condensate,

$$
\langle\bar{\psi} \psi\rangle=\left\langle\bar{\psi}_{R} \psi_{L}+\bar{\psi}_{L} \psi_{R}\right\rangle
$$

This condensate is not invariant (it mixes left and right) and therefore serves as an order parameter of the symmetry breaking. Note that in QCD the quark condensate is a renormalization scale dependent quantity. A recent estimate can be found in (Dosch and Narison 1998), with a numerical value,

$$
\langle 0|\bar{\psi} \psi| 0\rangle(1 \mathrm{GeV}) \simeq(-229 \mathrm{MeV})^{3} .
$$

The spontaneous breakdown of chiral symmetry thus implies that the (QCD) vacuum is nontrivial: it must contain quark-antiquark pairs with spins and momenta aligned in a way consistent with vacuum quantum numbers. A possible analog is the BCS ground state given in (207).

In terms of the full quark propagator,

$$
S(p)=\frac{\not p+M(p)}{p^{2}-M^{2}(p)},
$$

where we have allowed for a momentum dependent (or 'running') mass $M(p)$, the quark condensate is given by

$$
\langle 0|\bar{\psi} \psi| 0\rangle=-i \int \frac{d^{4} p}{(2 \pi)^{4}} \operatorname{tr} S(p)=-4 i N_{C} \int \frac{d^{4} p}{(2 \pi)^{4}} \frac{M(p)}{p^{2}-M^{2}(p)} .
$$

We thus see that the involved Dirac trace yields a nonvanishing condensate only if the effective quark mass $M(p)$ is nonzero. This links the existence of a quark condensate to the mechanism of dynamical mass generation. In this way we have found another argument that the bare quarks appearing in the QCD Hamiltonian (290) indeed acquire constituent masses. Of course we are still lacking a microscopic mechanism for that.

Goldstone's theorem (Goldstone 1961) now states that for any symmetry generator which does not leave the vacuum invariant, there must exist a massless boson with the quantum numbers of this generator. This results in the prediction 
Table 4. Masses of the pseudoscalar octet mesons (in $\mathrm{MeV}$ ).

\begin{tabular}{lccccc}
\hline \hline meson & $\pi^{0}$ & $\pi^{ \pm}$ & $K^{0}, \bar{K}^{0}$ & $K^{ \pm}$ & $\eta$ \\
\hline mass & 135 & 140 & 500 & 494 & 549 \\
\hline \hline
\end{tabular}

that in massless QCD one should have an octet of massless pseudoscalar mesons. In reality one finds what is listed in Table 4.

The nonvanishing masses of these mesons are interpreted as stemming from the nonvanishing quark masses in the QCD Hamiltonian which break chiral symmetry explicitly. Being small, they can be treated as perturbations.

It should be stressed that chiral symmetry has nothing to say about the mechanism of confinement which presumably is a totally different story. This is also reflected within a QCD based derivation of chiral symmetry breaking in terms of the instanton model, as presented e.g. in (Diakonov 1996). This model explains many facts of low-energy hadronic physics but is known not to yield confinement. It is therefore possible that confinement is not particularly relevant for the understanding of hadron structure (Diakonov 1996).

The instanton vacuum actually leads to an effective theory very close to the NJL model, the basics of which are our next topic.

\section{$5.2 \quad$ NJL Folklore}

Before I consider the light-cone formulation of the model, let me briefly recall its main physical features ${ }^{13}$. In its standard form, the NJL model has a chirally invariant four-fermion interaction, which can be imagined as the result of 'integrating out' the gluons in the QCD Lagrangian. For simplicity I concentrate on the case of one flavor. Extension to several flavors is straightforward. In the chiral limit (quark mass $m_{0}=0$ ), the Lagrangian is

$$
\mathcal{L}=\bar{\psi} i \not \partial \psi-G\left(\bar{\psi} \gamma_{\mu} \psi\right)^{2} \equiv \mathcal{L}_{0}+\mathcal{L}_{\text {int }} .
$$

Its four-fermion interaction is chirally symmetric under $U(1)_{L} \times U(1)_{R}$. We shall see in a moment that this symmetry is spontaneously broken.

It is important to observe that the coupling $G$ of the model has negative mass dimension, $[G]=-2$, hence, it is not renormalizable. Accordingly, it requires a cutoff which is viewed as a parameter of the model that is to be fixed by phenomenology. We thus follow the general spirit of effective field theory (Holstein 1995; Kaplan 1995; Manohar 1996). From the point of view of the light-cone

${ }_{13}$ For recent reviews, see (Vogl and Weise 1991; Klevansky 1992; Hatsuda and Kunihiro 1994). 
formulation to be developed later, the nonrenormalizability is an advantage: it enables us to 'circumvent' the difficulties of the light-cone renormalization program. There simply is no 'need' to renormalize.

Following the standard approach (Nambu and Jona-Lasinio 1961; Vogl and Weise 1991) we treat the model in mean-field approximation (which actually coincides with the large $-N$ limit). We begin with a Fierz transformation by schematically rewriting the interaction Lagrangian in Fierz symmetric form

$$
\mathcal{L}_{\text {int }}=G \sum_{i} c_{i}\left(\bar{\psi} \Gamma_{i} \psi\right)^{2}=G(\bar{\psi} \psi)^{2}+\ldots,
$$

where $i=S, P, V, A$ enumerates the different Dirac bilinears (scalar, pseudoscalar, vector and axial vector, respectively). In (298), I have only displayed the scalar part of the interaction because only the scalar density $S \equiv \bar{\psi} \psi$ can have a vacuum expectation value, the quark condensate,

$$
\langle S\rangle=\langle 0|\bar{\psi} \psi| 0\rangle .
$$

Let us determine this quantity in mean-field approximation. To define the latter we calculate,

$$
S^{2}=(S-\langle S\rangle+\langle S\rangle)^{2}=(S-\langle S\rangle)^{2}+2 S\langle S\rangle-\langle S\rangle^{2} \simeq 2 S\langle S\rangle+\text { const } .
$$

Thus, by neglecting quadratic fluctuations of $S$ around its expectation value, we linearize the interaction and obtain the mean-field Lagrangian,

$$
\mathcal{L}_{\mathrm{MFA}}=\bar{\psi}(i \not \partial+2 G\langle S\rangle) \psi .
$$

The mean-field solution has a very intuitive explanation. One essentially argues that the main effect of the interaction is to generate the mass of the quarks which become quasi-particles that interact only weakly. Neglecting this interaction entirely, one can view the process of mass generation as the transition of quarks with mass $m_{0}=0$ to mass $m$ resulting in a mass term $m \bar{\psi} \psi$ in the Lagrangian (301). We thus find that the dynamically generated mass is determined by the gap equation

$$
m=-2 G\langle 0|\bar{\psi} \psi| 0\rangle .
$$

How do we actually calculate the condensate $\langle 0|\bar{\psi} \psi| 0\rangle$ ? To this end we go back to (296) and express the condensate in terms of the full propagator 'at the origin', i.e. at space-time point $x=0$,

$$
\langle 0|\bar{\psi} \psi| 0\rangle_{m}=-i \operatorname{tr} S_{F}(x=0)=-i \operatorname{tr} \int \frac{d^{4} p}{(2 \pi)^{4}} \frac{1}{\not p-m+i \epsilon} .
$$

The following remarks are in order. First we note that within mean-field approximation the dynamically generated mass $m$ is constant, i.e. independent of the momentum $p$. Furthermore, the full propagator $S_{F}$ is defined in terms of the constituent mass $m$, so that the gap equation (302) becomes an implicit self-consistency condition where the mass $m$ to be determined appears on both 
sides. This equation will be solved in a moment. The integral appearing on the r.h.s. of (303) is quadratically divergent ${ }^{14}$ and has to be regulated. If we use a cutoff $\Lambda$ we have, for dimensional reasons,

$$
\langle 0|\bar{\psi} \psi| 0\rangle \sim \Lambda^{2} m .
$$

A particularly intuitive way to calculate the condensate (which will also be used in the light-cone case) is based on the Hellmann-Feynman theorem. This states that 'the derivative of an expectation value is the expectation value of the derivative', if the expectation is taken between normalizable states. If applied to the vacuum expectation value of our mean-field Hamiltonian,

$$
\mathcal{H}(m)=\mathcal{H}_{0}+m \bar{\psi} \psi
$$

which is the vacuum energy density $\mathcal{E}(m) \equiv\langle 0|\mathcal{H}(m)| 0\rangle$, the theorem yields,

$$
\langle 0|\bar{\psi} \psi| 0\rangle_{m}=\frac{\partial}{\partial m} \mathcal{E}(m)=-\frac{\partial}{\partial m} \int \frac{d^{3} p}{(2 \pi)^{3}} 2 \sqrt{\mathbf{p}^{2}+m^{2}} .
$$

The factor of two in the integrand is due to the fermion spin degeneracy. If we choose a noncovariant three-vector cutoff, $|\mathbf{p}|<\Lambda_{3}$, the result for the condensate is of the form (304),

$$
\langle 0|\bar{\psi} \psi| 0\rangle_{m}=-\frac{m \Lambda_{3}^{2}}{2 \pi^{2}}\left[1+\frac{\delta^{2}}{2} \ln \delta^{2}+O\left(\delta^{2}\right)\right],
$$

with $\delta \equiv m / \Lambda_{3}$. The dynamical mass is found by inserting this result into the gap equation (302). A nontrivial solution $m=m(G) \neq 0$ arises above a critical coupling $G_{c}$ which is determined by the identity $m\left(G_{c}\right)=0$. It is known from the theory of critical phenomena that the mean-field approximation leads to a square root behaviour of the mass around $G_{c}=4 \pi^{2} / \Lambda^{2}$,

$$
m(G) \sim\left(G-G_{c}\right)^{1 / 2}, \quad G \geq G_{c} .
$$

We thus have seen that the NJL model describes the transformation of bare quarks of mass $m_{0}(=0)$ into dressed (or constituent) quarks $(Q)$ of mass $m \neq$ 0 . By considering the bound state equation in the pseudoscalar channel one can also verify Goldstone's mechanism: there is a massless $Q \bar{Q}$ bound state, which is identified with the pion, exactly if the gap equation holds (Nambu and Jona-Lasinio 1961; Vogl and Weise 1991). This concludes the presentation of spontaneous chiral symmetry breaking within the model. One should keep in mind that it follows the same pattern as in QCD.

Let me conclude the NJL 'crash course' by reemphasizing that the model does not confine. This means in particular, that there is a nonvanishing probability for mesons to decay into constituent quarks. We thus cannot expect to obtain

${ }^{14}$ As the condensate involves the product of field operators at coinciding space-time points, this clearly is a short-distance singularity. 
reliable estimates for strong decay widths of mesons or other quantities that are not dominated by their chiral properties.

It is getting time to discuss the light-cone formulation of the model. For a 'light-cone physicist' the model is interesting for several reasons. I have already pointed out that the lack of renormalizability is welcome because we only have to worry about a proper regularization. Furthermore, the model addresses the conceptually important questions of spontaneous symmetry breaking and condensates. Are these in conflict with a trivial vacuum? Finally, a constituent picture seems to be realized which should make a truncation of the light-cone Schrödinger equation feasible. Now, we again encounter the standard rule of physics that there is nothing like free lunch. Here, this is mainly due to the appearance of complicated constraints for part of the fermionic degrees of freedom. Let me thus make a small aside on the special features of light-cone fermions.

Light-Cone Fermions. The solution of the Dirac equation (for free fermions of mass $m_{0}$ ) has the following light-cone Fock expansion at $x^{+}=0$,

$$
\psi(\boldsymbol{x}, 0)=\sum_{\lambda} \int_{0}^{\infty} \frac{d k^{+}}{k^{+}} \int \frac{d^{2} k_{\perp}}{16 \pi^{3}}\left[b(\boldsymbol{k}, \lambda) u(\boldsymbol{k}, \lambda) e^{-i \boldsymbol{k} \cdot \boldsymbol{x}}+d^{\dagger}(\boldsymbol{k}, \lambda) v(\boldsymbol{k}, \lambda) e^{i \boldsymbol{k} \cdot \boldsymbol{x}}\right],
$$

where we recall the notations

$$
\boldsymbol{k} \equiv\left(k^{+}, \mathbf{k}_{\perp}\right), \quad \boldsymbol{x} \equiv\left(x^{-}, \mathbf{x}_{\perp}\right), \quad \boldsymbol{k} \cdot \boldsymbol{x} \equiv \frac{1}{2} k^{+} x^{-}-\mathbf{k}_{\perp} \cdot \mathbf{x}_{\perp} .
$$

Like for scalars, the Fock measure is independent of the mass $m_{0}$. The Fock operators satisfy the canonical anti-commutation relations,

$$
\left\{b(\boldsymbol{k}, \lambda), b^{\dagger}\left(\boldsymbol{p}, \lambda^{\prime}\right)\right\}=\left\{d(\boldsymbol{k}, \lambda), d^{\dagger}\left(\boldsymbol{p}, \lambda^{\prime}\right)\right\}=16 \pi^{3} k^{+} \delta^{3}(\boldsymbol{k}-\boldsymbol{p}),
$$

The basis spinors $u$ and $v$ obey the Dirac equations,

$$
\begin{aligned}
& \left(\not k-m_{0}\right) u(\boldsymbol{k}, \lambda)=0, \\
& \left(\not k+m_{0}\right) v(\boldsymbol{k}, \lambda)=0,
\end{aligned}
$$

and are explicitly given by

$$
\begin{aligned}
u(\boldsymbol{k}, \lambda) & =\frac{1}{\sqrt{k^{+}}}\left(k^{+}+\beta m+\alpha_{i} k_{i}\right) X_{\lambda}, \\
v(\boldsymbol{k}, \lambda) & =\frac{1}{\sqrt{k^{+}}}\left(k^{+}-\beta m+\alpha_{i} k_{i}\right) X_{-\lambda} .
\end{aligned}
$$

The four-spinor $X$ will be defined in a moment; $\alpha_{i}$ and $\beta$ are the standard hermitean Dirac matrices. The crucial point is the decomposition of the fermion field, $\psi=\psi_{+}+\psi_{-}$into 'good' (+) and 'bad' (-) components, $\psi_{ \pm} \equiv \Lambda_{ \pm} \psi$, by means of the projection matrices,

$$
\Lambda_{ \pm} \equiv \frac{1}{4} \gamma^{\mp} \gamma^{ \pm}=\frac{1}{2}\left(\begin{array}{cc}
\mathbb{1} & \pm \sigma_{3} \\
\pm \sigma_{3} & \mathbb{1}
\end{array}\right)
$$


The spinor $X$ appearing in (314) and (315) is an eigenspinor of $\Lambda_{+}, \Lambda_{+} X_{\lambda}=X_{\lambda}$. The Dirac equation decomposes accordingly into two equations. The one for $\psi_{-}$ reads

$$
2 i \partial^{+} \psi_{-}=\left(-i \gamma^{i} \partial^{i}+m_{0}\right) \gamma^{+} \psi_{+},
$$

and does not contain a light-cone time derivative. Therefore, the bad component $\psi_{-}$is constrained; it can be expressed in terms of the good component, i.e. $\psi_{-}=$ $\psi_{-}\left[\psi_{+}\right]$. Again, this requires the inversion of the notorious spatial derivative $\partial^{+}$. As a result, the free Dirac Hamiltonian (density) only depends on $\psi_{+}$,

$$
\mathcal{H}=\psi_{+}^{\dagger} \frac{-\partial_{\perp}^{2}+m_{0}^{2}}{i \partial^{+}} \psi_{+} \equiv \mathcal{H}\left[\psi_{+}\right]
$$

where we easily recognize the light-cone energy, $\left(k_{\perp}^{2}+m_{0}^{2}\right) / k^{+}$.

It turns out that in case of a four-fermion interaction like in the NJL model the constraint becomes rather awkward to solve. In particular, its solution has to be consistent with the mean field approximation employed (Dietmaier et al. 1989). This can be achieved most elegantly by using the large $-N$ expansion (Bentz et al. 1999; Itakura and Maedan 2000). Nevertheless, the light-cone Hamiltonian of the model is a rather complicated expression. We will therefore follow an alternative road which is the topic of the next subsection.

\subsection{Schwinger-Dyson Approach}

The first derivation, analysis and solution of a light-cone bound-state equation appeared in 't Hooft's original paper on what is now called the 't Hooft model ('t Hooft 1974). We have discussed this model in the last section where we also rederived 't Hooft's solution. Interestingly, 't Hooft did not use the light-cone formalism in the manner we presented it and which nowadays might be called standard. This amounts to deriving the canonical light-cone Hamiltonian and setting up the associated system of bound-state equations by projecting on the different sectors of Fock space (cf. Section 4). Instead, he started from covariant equations, namely the Schwinger-Dyson equations for the quark propagator (or self-energy), and the Bethe-Salpeter equation for the bound-state amplitude, which needs the quark self-energy as an input. The light-cone Schrödinger equation was then obtained by projecting the Bethe-Salpeter equation onto hypersurfaces of equal light-cone time. In this way, one avoids to explicitly derive the light-cone Hamiltonian, which, as explained above, can be a tedious enterprise in view of complicated constraints one has to solve. Let us therefore have a closer look at this way of proceeding.

The Schwinger-Dyson Equation for the Propagator. The first step in the program ${ }^{15}$ is to solve the Schwinger-Dyson equation for the propagator,

$\overline{15}$ For recent literature on the Schwinger-Dyson approach, see e.g. (Roberts and Williams 1994; Roberts and Schmidt 2000; Maris and Tandy 2000). 
or, equivalently, for the quark self-energy. As this cannot be done exactly, one resorts to mean-field (or large $-N$ ) approximation. This is essentially what has been done in the last subsection. Let us rewrite this in terms of Schwinger-Dyson equations. The one for the full propagator $S$ reads

$$
S=S_{0}+S_{0} \Sigma S,
$$

and is formally solved by

$$
S(p)=\frac{1}{S_{0}^{-1}(p)-\Sigma(p)},
$$

where $S_{0}$ is the free propagator,

$$
S_{0}(p)=\frac{1}{p-m_{0}},
$$

and $\Sigma$ the quark self-energy. In mean-field approximation, it is momentum independent and defines the constituent mass through the gap equation, $\Sigma=$ const $=m$, see (302) and (303).

To solve the latter in the light-cone framework, we basically just have to calculate the condensate. As in the standard approach, this can be obtained via the Feynman-Hellman theorem by differentiating the energy density of the quasi-particle Dirac sea,

$$
\begin{aligned}
\langle 0|\bar{\psi} \psi| 0\rangle_{m} & =\frac{\partial}{\partial m} \mathcal{E}(m)=\frac{\partial}{\partial m} \int_{-\infty}^{0} d k^{+} \int \frac{d^{2} k_{\perp}}{16 \pi^{3}} 2 \frac{m^{2}+k_{\perp}^{2}}{k^{+}} \\
& =-\frac{m}{4 \pi^{3}} \int_{0}^{\infty} \frac{d k^{+}}{k^{+}} \int d^{2} k_{\perp},
\end{aligned}
$$

Again, as it stands, the integral is divergent and requires regularization. In the most straightforward manner one might choose $m^{2} / \Lambda \leq k^{+} \leq \Lambda$ and $\left|\mathbf{k}_{\perp}\right| \leq \Lambda$, so that the condensate becomes

$$
\langle 0|\bar{\psi} \psi| 0\rangle_{m}=-\frac{m}{4 \pi^{2}} \int_{m^{2} / \Lambda}^{\Lambda} \frac{d k^{+}}{k^{+}} \int_{0}^{\Lambda^{2}} d\left(k_{\perp}^{2}\right)=-\frac{m}{4 \pi^{2}} \Lambda^{2} \ln \frac{\Lambda^{2}}{m^{2}} .
$$

Plugging this result into the gap equation (302) one finds for the dynamical mass squared,

$$
m^{2}(G)=\Lambda^{2} \exp \left(-\frac{2 \pi^{2}}{G \Lambda^{2}}\right) .
$$

The critical coupling is determined by the vanishing of this mass, $m\left(G_{c}\right)=0$, and from (324) we find the surprising result

$$
G_{c}=0 \text {. }
$$


This result, however, is wrong since one knows from the conventional treatment of the model that the critical coupling is finite of the order $\pi^{2} / \Lambda^{2}$, both for covariant and noncovariant cutoff (Nambu and Jona-Lasinio 1961). In addition, it is quite generally clear that in the free theory $(G=0)$ chiral symmetry is not broken (as $m_{0}=0$ ) and, therefore, this should not happen for arbitrarily small coupling, either, cf. (308). The remedy is to use the invariant-mass cutoff (Lepage and Brodsky 1980),

$$
M_{0}^{2} \equiv \frac{k_{\perp}^{2}+m^{2}}{x(1-x)} \leq \Lambda^{2},
$$

where we have defined the longitudinal momentum fraction, $x \equiv k^{+} / \Lambda$. This provides a cutoff both in $x\left(\right.$ or $\left.k^{+}\right)$and $k_{\perp}$,

$$
\begin{aligned}
0 & \leq k_{\perp}^{2} \leq \Lambda^{2} x(1-x)-m^{2}, \\
x_{0} & \leq x \leq x_{1}, \\
x_{0,1} & \equiv \frac{1}{2}\left(1 \mp \sqrt{1-4 \epsilon^{2}}\right),
\end{aligned}
$$

with $\epsilon^{2} \equiv m^{2} / \Lambda^{2}$. Note that the transverse cutoff becomes a polynomial in $x$. The $k_{\perp}$-integration thus has to be performed before the $x$-integration.

For the condensate (322) the invariant-mass cutoff (326) yields an analytic structure different from (323),

$$
\langle 0|\bar{\psi} \psi| 0\rangle_{m}=-\frac{m \Lambda^{2}}{8 \pi^{2}}\left(1+2 \epsilon^{2} \ln \epsilon^{2}+O\left(\epsilon^{2}\right)\right),
$$

where we have neglected sub-leading terms in $\epsilon^{2}$. The result (330) coincides with the standard one, (307), if one identifies the noncovariant cutoffs according to

$$
\Lambda^{2} \equiv 4\left(\Lambda_{3}^{2}+m^{2}\right)
$$

This has independently been observed by (Bentz et al. 1999). From (330), one infers the correct cutoff dependence of the critical coupling,

$$
G_{c}=\frac{4 \pi^{2}}{\Lambda^{2}} .
$$

The moral of this calculation is that even in a nonrenormalizable theory like the NJL model, the light-cone regularization prescription is a subtle issue.

In the NJL model with its second-order phase transition of mean-field type, the usual analogy with magnetic systems can be made. Chiral symmetry corresponds to rotational symmetry, the vacuum energy density to the Gibbs free energy, and the mass $m$ to an external magnetic field. The order parameter measuring the rotational symmetry breaking is the magnetization. It is obtained by differentiating the free energy with respect to the external field. This is the analogue of expression (322) derived from the Feynman-Hellmann theorem. 
The Bound-State Equation. Once the physical fermion mass $m$ is known by solving the gap equation, it can be plugged into the Bethe-Salpeter equation for quark-antiquark bound states (mesons), given by

$$
\chi_{\mathrm{BS}}=S_{1} S_{2} K \chi_{\mathrm{BS}} .
$$

$S_{1}, S_{2}$ denote the full propagators of quark and anti-quark, $K$ the BetheSalpeter kernel, and $\chi_{\mathrm{BS}}$ the Bethe-Salpeter amplitude. From the latter, one obtains the light-cone wave function via integration over the energy variable $k^{-}$ (Michael and Payne 1982; Brodsky et al. 1985; Chakrabarty et al. 1989; Liu and Soper 1993),

$$
\phi_{\mathrm{LC}}(\boldsymbol{k})=\int \frac{d k^{-}}{2 \pi} \chi_{\mathrm{BS}}(k), \quad \boldsymbol{k}=\left(k^{+}, \mathbf{k}_{\perp}\right) .
$$

In ladder approximation (again equivalent to the large $-N$ limit), (333) and (334) become

$$
\phi_{\mathrm{LC}}(\boldsymbol{k})=\int \frac{d k^{-}}{2 \pi} S(k) S(k-P) \int \frac{d^{3} p}{(2 \pi)^{3}} \int \frac{d p^{-}}{2 \pi} K(k, p) \chi_{\mathrm{BS}}(p),
$$

with $P$ denoting the bound-state four-momentum. On the left-hand-side, the projection onto $x^{+}=0$ (i.e. the $k^{-}$-integration) has already been carried out. On the right-hand-side, the two integrations over $k^{-}$and $p^{-}$still have to be performed. Whether this can easily be done depends of course crucially on the kernel $K$, which in principle is a function of both energy variables. For the NJL model, however, $K$ assumes the very simple form,

$$
K(k, p)=2 \gamma_{5} \otimes \gamma_{5}-\gamma_{\mu} \gamma_{5} \otimes \gamma^{\mu} \gamma_{5},
$$

i.e. it is momentum independent due to the four-point contact interaction,

$$
W \sim \int d^{4} x \int d^{4} y(\bar{\psi} \Gamma \psi)(x) \delta^{4}(x-y)(\bar{\psi} \Gamma \psi)(y) .
$$

Thus, the $p^{-}$-integration immediately yields $\phi_{\mathrm{LC}}$, and the $k^{-}$-integration can be performed via residue techniques and is completely determined by the poles of the propagators, $S(k)$ and $S(k-P)$. As a result, one finds a nonvanishing result only if $0 \leq k^{+} \leq P^{+}$, and one of the two particles is put on-shell, e.g. $k^{2}=m^{2}$, as already observed by (Gross 1989).

The upshot of all this is nothing but the light-cone bound-state equation, which explicitly reads

$$
\begin{aligned}
\phi_{\mathrm{LC}}\left(x, \mathbf{k}_{\perp}\right) & =-\frac{2 G}{x(1-x)} \frac{(\hat{\not k}+m) \gamma_{5}(\hat{\not k}-\not P+m)}{M^{2}-M_{0}^{2}} \\
& \times \int_{0}^{1} d y \int \frac{d^{2} p_{\perp}}{8 \pi^{3}} \operatorname{tr}\left[\gamma_{5} \phi_{\mathrm{LC}}\left(y, \mathbf{p}_{\perp}\right)\right] \theta_{\Lambda}\left(y, \mathbf{p}_{\perp}\right) \\
& +\frac{G}{x(1-x)} \frac{(\hat{\not k}+m) \gamma_{\mu} \gamma_{5}(\hat{\not k}-\not P+m)}{M^{2}-M_{0}^{2}}
\end{aligned}
$$




$$
\times \int_{0}^{1} d y \int \frac{d^{2} p_{\perp}}{8 \pi^{3}} \operatorname{tr}\left[\gamma^{\mu} \gamma_{5} \phi_{\mathrm{LC}}\left(y, \mathbf{p}_{\perp}\right)\right] \theta_{\Lambda}\left(y, \mathbf{p}_{\perp}\right) .
$$

Here we have defined the longitudinal momentum fractions $x=k^{+} / P^{+}, y=$ $p^{+} / P^{+}$, the on-shell momentum $\hat{k}=\left(\hat{k}^{-}, \mathbf{k}_{\perp}, k^{+}\right)$with $\hat{k}^{-}=\left(k_{\perp}^{2}+m^{2}\right) / k^{+}$, and the bound state mass squared, $M^{2}=P^{2}$, which is the eigenvalue to be solved for. $\theta_{\Lambda}\left(x, \mathbf{p}_{\perp}\right)$ denotes the invariant mass cutoff $(326)$.

Now, while (338) may appear somewhat complicated it is actually very simple; indeed, it is basically already the solution of the problem. The crucial observation is to note that the two integral expressions are mere normalization constants,

$$
\begin{aligned}
C_{\Lambda} & \equiv \int_{0}^{1} d y \int \frac{d^{2} p_{\perp}}{8 \pi^{3}} \operatorname{tr}\left[\gamma_{5} \phi_{\mathrm{LC}}\left(y, \mathbf{p}_{\perp}\right)\right] \theta_{\Lambda}\left(y, \mathbf{p}_{\perp}\right), \\
D_{\Lambda} P^{\mu} & \equiv \int_{0}^{1} d y \int \frac{d^{2} p_{\perp}}{8 \pi^{3}} \operatorname{tr}\left[\gamma^{\mu} \gamma_{5} \phi_{\mathrm{LC}}\left(y, \mathbf{p}_{\perp}\right)\right] \theta_{\Lambda}\left(y, \mathbf{p}_{\perp}\right) .
\end{aligned}
$$

Thus, the solution of the light-cone bound-state equation (338) is

$$
\begin{aligned}
\phi_{\mathrm{LC}}\left(x, \mathbf{k}_{\perp}\right) & =-\frac{2 G C_{\Lambda}}{x(1-x)} \frac{(\hat{\not k}+m) \gamma_{5}(\hat{\not k}-\not P+m)}{M^{2}-M_{0}^{2}} \\
& +\frac{G D_{\Lambda}}{x(1-x)} \frac{(\hat{\not k}+m) \not P \gamma_{5}(\hat{\not k}-\not P+m)}{M^{2}-M_{0}^{2}},
\end{aligned}
$$

with yet undetermined normalization constants $C_{\Lambda}$ and $D_{\Lambda}$. As a first check of our bound-state wave function (341) we look for a massless pion in the chiral limit. To this end we decompose the light-cone wave function into Dirac components according to (Lucha et al. 1991),

$$
\phi_{\mathrm{LC}}=\phi_{\mathrm{S}}+\phi_{\mathrm{P}} \gamma_{5}+\phi_{\mathrm{A}}^{\mu} \gamma_{\mu} \gamma_{5}+\phi_{\mathrm{V}}^{\mu} \gamma_{\mu}+\phi_{\mathrm{T}}^{\mu \nu} \sigma_{\mu \nu}
$$

Multiplying (341) with $\gamma_{5}$, taking the trace and integrating over $\boldsymbol{k}$ we find

$$
\begin{aligned}
C_{\Lambda} & =-\frac{G C_{\Lambda}}{2 \pi^{3}} \int_{0}^{1} d x \int d^{2} k_{\perp} \frac{M^{2} x+M_{0}^{2}(1-x)}{x(1-x)\left(M^{2}-M_{0}^{2}\right)} \theta_{\Lambda}\left(x, \mathbf{k}_{\perp}\right) \\
& +\frac{G D_{\Lambda}}{2 \pi^{3}} M^{2} \int_{0}^{1} d x \int d^{2} k_{\perp} \theta_{\Lambda}\left(x, \mathbf{k}_{\perp}\right) .
\end{aligned}
$$

In the chiral limit one expects a solution for $M=0$, the Goldstone pion. In this case one obtains

$$
1=\frac{G}{2 \pi^{3}} \int_{0}^{1} d x \int d^{2} k_{\perp} \theta_{\Lambda}\left(x, \mathbf{k}_{\perp}\right) .
$$

This is exactly the gap equation (302) using the definition (322) of the condensate (with the invariant-mass cutoff (326) understood in both identities). Note once more the light-cone peculiarity that the (Fock) measure in (344) is entirely 
mass independent. All the mass dependence, therefore, has to come from the (invariant-mass) cutoff. Otherwise one will get a wrong behavior of the dynamical mass $m$ as a function of the coupling $G$, as was the case in (324).

With this in mind, we see that the Goldstone pion is a solution of the lightcone bound-state equation exactly if the gap equation holds. This provides additional evidence for the self-consistency of the procedure. The deeper reason for the fact that the quark self-energy and the bound-state amplitude satisfy essentially the same equation, is the chiral Ward identity relating the quark propagator and the pseudoscalar vertex (Savklı and Tabakin 1998).

Our next task is to actually evaluate the solution (341) of the bound-state equation. $\phi_{\mathrm{LC}}$ is a Dirac matrix and therefore is not yet a light-cone wave function as defined in Section 4. The relation between the two quantities has been given by (Liu and Soper 1993),

$$
2 P^{+} \psi\left(x, \mathbf{k}_{\perp}, \lambda, \lambda^{\prime}\right)=\bar{u}\left(x P^{+}, \mathbf{k}_{\perp}, \lambda\right) \gamma^{+} \phi_{\mathrm{LC}}(\boldsymbol{k}) \gamma^{+} v\left(\bar{x} P^{+},-\mathbf{k}_{\perp}, \lambda^{\prime}\right),
$$

where we have denoted $\bar{x} \equiv 1-x$ to save space. A somewhat lengthy calculation yields the result (Heinzl 1998),

$$
\psi\left(x, \mathbf{k}_{\perp}, \lambda, \lambda^{\prime}\right)=\frac{2 G P^{+} / \sqrt{x \bar{x}}}{M^{2}-M_{0}^{2}}\left(\frac{2 C_{\Lambda}}{M} \bar{u}_{\lambda} M \gamma_{5} v_{\lambda^{\prime}}-D_{\Lambda} \bar{u}_{\lambda} \not P \gamma_{5} v_{\lambda^{\prime}}\right),
$$

with the arguments of the spinors $\bar{u}$ and $v$ suppressed. At this point we have to invoke another symmetry principle. (Ji et al. 1992) have pointed out that the spin structure $(\bar{u} \Gamma v)$ should be consistent with the one obtained form the instant form spinors via a subsequent application of a Melosh transformation (Melosh 1974) and a boost. Using this recipe, one obtains the following relation between the constants $C_{\Lambda}$ and $D_{\Lambda}$,

$$
2 C_{\Lambda} / M=-D_{\Lambda} \equiv N / 2 G .
$$

As a result, the spin structure in (346) coincides with the standard one used e.g. in (Dziembowski 1988; Chung et al. 1988; Jaus 1990; Ji and Cotanch 1990; Ji et al. 1992). The NJL wave function of the pion thus becomes

$$
\psi\left(x, \mathbf{k}_{\perp}, \lambda, \lambda^{\prime}\right)=\frac{N P^{+} / \sqrt{x \bar{x}}}{M^{2}-M_{0}^{2}} \bar{u}_{\lambda}(M+\not P) \gamma_{5} v_{\lambda^{\prime}} \theta\left(\Lambda^{2}-M_{0}^{2}\right) .
$$

Not surprisingly, the off-shellness $M^{2}-M_{0}^{2}$ appears in the denominator. $N$ is the normalization parameter defined in (347), and the spin (or helicity) structure is given by

$$
\begin{aligned}
& \bar{u}\left(x P^{+}, \mathbf{k}_{\perp}, \lambda\right)(M+\not P) \gamma_{5} v\left(\bar{x} P^{+},-\mathbf{k}_{\perp}, \lambda^{\prime}\right)= \\
& =\frac{1}{\sqrt{x \bar{x}} P^{+}}\left[\lambda\left(m M+m^{2}-\mathbf{k}_{\perp}^{2}+M^{2} x \bar{x}\right) \delta_{\lambda,-\lambda^{\prime}}-k_{-\lambda}(M+2 m) \delta_{\lambda \lambda^{\prime}}\right]
\end{aligned}
$$

where we have used (314), (315) and denoted $k_{\lambda} \equiv k^{1}+i \lambda k^{2}$. The first term with spins anti-parallel corresponds to $L_{z}=0$, the second one (with spins parallel) to 
$L_{z}= \pm 1$. It has already been pointed out by Leutwyler that both spin alignments should contribute to the pion wave function (Leutwyler 1974a; Leutwyler 1974b). Note that the latter is a cutoff dependent quantity. This is necessary in order to render the wave function normalizable. A single power of the off-shellness in the denominator is not sufficient for that. Only the cutoff guarantees the boundary conditions (251) so that the wave function drops off sufficiently fast in $x$ and $k_{\perp}$.

As we are interested in analyzing the quality of a constituent picture, we approximate the pion by its valence state, denoting $\psi \equiv \psi_{2}$,

$$
|\pi: \boldsymbol{P}\rangle=\sum_{\lambda, \lambda^{\prime}} \int_{0}^{1} d x \int \frac{d^{2} k_{\perp}}{16 \pi^{3}} \psi_{2}\left(x, \mathbf{k}_{\perp}, \lambda, \lambda^{\prime}\right)\left|q \bar{q}: x, \mathbf{k}_{\perp}, \lambda, \lambda^{\prime}\right\rangle
$$

which should be compared with the general expression (240). The normalization of this state is given by (237),

$$
\left\langle\pi: \boldsymbol{P}^{\prime} \mid \pi: \boldsymbol{P}\right\rangle=16 \pi^{3} P^{+} \delta^{3}\left(\boldsymbol{P}-\boldsymbol{P}^{\prime}\right) .
$$

As usual we work in a frame in which the total transverse momentum vanishes, i.e. $\boldsymbol{P}=\left(P^{+}, \mathbf{P}_{\perp}=0\right)$. Expression (351) yields the normalization (247) of the wave function,

$$
\sum_{\lambda \lambda^{\prime}} \int_{0}^{1} d x \int \frac{d^{2} k_{\perp}}{16 \pi^{3}}\left|\psi_{2}\left(x, \mathbf{k}_{\perp}, \lambda, \lambda^{\prime}\right)\right|^{2} \equiv\left\|\psi_{2}\right\|^{2}=1 .
$$

It is of course a critical assumption that the probability to find the pion in its valence state is one. In this way we enforce a constituent picture by fiat, and it is clear that such an assumption has to be checked explicitly by comparing with phenomenology.

\subsection{Observables}

With the light-cone wave function at hand, we are in the position to calculate observables. To proceed we will employ the following two simplifications. First of all, we will always work in the chiral limit of vanishing quark mass, $m_{0}=0$, which, as we have seen, leads to a massless Goldstone pion, $M=0$. We write the pion wave function as a matrix in helicity space,

$$
\begin{aligned}
\psi_{2}\left(x, \mathbf{k}_{\perp}\right) & =\left(\begin{array}{c}
\psi_{2 \uparrow \uparrow} \psi_{2 \uparrow \downarrow} \\
\psi_{2 \downarrow \uparrow} \psi_{2 \downarrow \downarrow}
\end{array}\right) \\
& =-\frac{N}{k_{\perp}^{2}+m^{2}}\left(\begin{array}{cc}
-2 m\left(k^{1}-i k^{2}\right) & m^{2}-k_{\perp}^{2} \\
k_{\perp}^{2}-m^{2} & -2 m\left(k^{1}+i k^{2}\right)
\end{array}\right) \theta\left(\Lambda^{2}-M_{0}^{2}\right) .
\end{aligned}
$$

Note that, in the chiral limit, the wave function becomes independent of $x$ (apart from cutoff effects). This actually agrees with our findings in the two-dimensional 't Hooft model, cf. (276). 
The diagonal terms in (353) correspond to parallel spins, the off-diagonal ones to anti-parallel spins. The different components are related by the symmetry properties,

$$
\psi_{2 \downarrow \downarrow}=\psi_{2 \uparrow \uparrow}^{*}, \quad \psi_{2 \downarrow \uparrow}=-\psi_{2 \uparrow \downarrow} .
$$

Second, we will go to the large-cutoff limit, that is, we will keep only the leading order in $\epsilon^{2}=m^{2} / \Lambda^{2}$. We thus assume that the cutoff is large compared to the constituent mass. From the standard values, $\Lambda \simeq 1 \mathrm{GeV}, m \simeq 300 \mathrm{MeV}$, we expect that this assumption should induce an error of the order of $10 \%$. The technical advantage of the large-cutoff limit is a simple analytic evaluation of all the integrals we will encounter. Furthermore, the leading order will be independent of the actual value of the constituent mass. It should be mentioned that the same procedure has been used in calculations based on the instanton model of the QCD vacuum (Petrov et al. 1999). There, the ratio $\epsilon^{2}$ can be related to parameters of the instanton vacuum, namely

$$
\epsilon^{2}=(m \rho)^{2} \sim(\rho / R)^{4},
$$

where $\rho \simeq 1 / 3 \mathrm{fm}$ is the instanton size and $R$ the mean distance between instantons. Thus, $\epsilon^{2}$ can be identified with the 'diluteness parameter' or 'packing fraction' of the instanton vacuum and hence is parametrically small, $\epsilon^{2} \simeq 1 / 4$.

The upshot of all this is that we work with the extremely simple model wave function (Radyushkin 1995)

$$
\psi_{2 \uparrow \downarrow} \simeq N \theta\left(\Lambda^{2}-M_{0}^{2}\right), \quad \psi_{2 \uparrow \uparrow}=0,
$$

which is entirely determined by two parameters, the normalization constant $N$ and the cutoff $\Lambda$. We thus need two constraints on the wave function to fix our two parameters.

Normalization. As announced, we enforce a constituent picture by demanding (352) which decomposes into

$$
1=\left\|\psi_{2}\right\|^{2}=\left\|\psi_{2 \uparrow \downarrow}\right\|^{2}+\left\|\psi_{2 \uparrow \uparrow}\right\|^{2}+\left\|\psi_{2 \downarrow \uparrow}\right\|^{2}+\left\|\psi_{2 \downarrow \downarrow}\right\|^{2} .
$$

Explicitly, one finds

$$
\begin{aligned}
\left\|\psi_{2 \uparrow \downarrow}\right\|^{2} & =\left\|\psi_{2 \downarrow \uparrow}\right\|^{2}=N^{2} \int_{0}^{1} d x \int \frac{d^{2} k_{\perp}}{16 \pi^{3}} \theta\left(\Lambda^{2}-M_{0}^{2}\right) \\
& =\frac{N^{2}}{16 \pi^{2}} \int_{0}^{1} d x \int_{0}^{\Lambda^{2} x(1-x)} d k_{\perp}^{2}=\frac{N^{2} \Lambda^{2}}{96 \pi^{2}} \stackrel{!}{=} 1 / 2,
\end{aligned}
$$

while the components with parallel spins have vanishing norm in the large-cutoff limit, $\left\|\psi_{2 \uparrow \uparrow}\right\|^{2}=\left\|\psi_{2 \downarrow \downarrow}\right\|^{2}=0$, cf. (356). 
Pion Decay Constant. A second constraint on the wave function is provided by the pion decay constant $f_{\pi}$, which appears in the semi-leptonic process $\pi \rightarrow$ $\mu \nu$. The relevant matrix element is

$$
\left\langle 0\left|\bar{\psi}_{\bar{d}}(0) \gamma^{+} \gamma_{5} \psi_{u}(0)\right| \pi^{+}\left(P^{+}\right)\right\rangle=i \sqrt{2} P^{+} f_{\pi},
$$

$\bar{\psi}_{\bar{d}}$ and $\psi_{u}$ denote the field operators of the $\bar{d}$ and $u$ quark in the pion. If we insert all quantum numbers, the pion state to the right of the matrix element is given by

$$
\left|\pi^{+}\right\rangle=\psi_{\bar{d} u} \otimes \frac{1}{\sqrt{6}}\left(\left|\bar{d}_{c \uparrow} u_{c \downarrow}\right\rangle-\left|\bar{d}_{c \downarrow} u_{c \uparrow}\right\rangle\right) .
$$

The spatial (or internal) structure of the state is encoded in the light-cone wave function $\psi_{2} \equiv \psi_{\bar{d} u}$. If we insert the Fock expansions (309) for $\bar{\psi}_{\bar{d}}$ and $\psi_{u}$ as well as the pion state (350), we obtain the following constraint on the pion wave function,

$$
\int_{0}^{1} d x \int \frac{d^{2} k_{\perp}}{16 \pi^{3}} \psi_{2 \uparrow \downarrow}\left(x, \mathbf{k}_{\perp}\right)=\frac{f_{\pi}}{2 \sqrt{3}} .
$$

The left-hand-side is basically the (position space) 'wave function at the origin'. Quark $(u)$ and antiquark $(\bar{d})$ thus have to sit on top of each other in order to have sizable probability for decay. Note that only the $L_{z}=0$ component contributes. Concerning the effect of higher Fock states, it can be shown (Lepage et al. 1981; Brodsky and Lepage 1989) that indeed only the valence wave function contributes to (361). This constraint is therefore exact and holds beyond a constituent picture. Empirically, the pion decay constant is $f_{\pi}=92.4 \mathrm{MeV}$ (Holstein 1995).

As already stated, this is our second source of phenomenological information to fix cutoff and normalization. Using the explicit form (356) of the wave function, the constraint (361) becomes

$$
\int_{0}^{1} d x \int \frac{d^{2} k_{\perp}}{16 \pi^{3}} \psi_{2 \uparrow \downarrow}\left(x, \mathbf{k}_{\perp}\right)=\frac{N \Lambda^{2}}{96 \pi^{2}} \stackrel{!}{=} \frac{f_{\pi}}{2 \sqrt{3}} .
$$

With (362) and (358) we now have two equations for our two parameters which accordingly are determined as

$$
\begin{aligned}
N & =\sqrt{3} / f_{\pi}, \\
\Lambda & =4 \pi f_{\pi} \simeq 1.16 \mathrm{GeV} .
\end{aligned}
$$

The value (364) for the cutoff $\Lambda$ is the standard scale below which chiral effective Lagrangians are believed to make sense (Manohar and Georgi 1984). It is reassuring that within our approximations we get exactly this value. This means that we are not doing something entirely stupid. A more severe test of consistency is provided by the next constraint to be satisfied. 
Constraint from $\boldsymbol{\pi}^{\mathbf{0}} \rightarrow \mathbf{2 \gamma}$. This constraint has also been derived by Brodsky and Lepage (Lepage et al. 1981) within an analysis of the $\pi \gamma$ transition form factor. It assumes the very simple form,

$$
\int_{0}^{1} d x \psi_{2 \uparrow \downarrow}\left(x, \mathbf{0}_{\perp}\right)=\frac{\sqrt{3}}{f_{\pi}} .
$$

Inserting the light-cone wave function (356), the right-hand-side simply becomes the normalization $N$ which is indeed consistent with our findings (363) and (364). We mention in passing that the constraint (365) usually is the simplest way to fix the normalization $N$. Its derivation, however, is more complicated than that of (361).

Pion Form Factor. We proceed by calculating the pion electromagnetic formfactor. It is defined by the matrix element of the electromagnetic current $J_{\mathrm{em}}^{\mu}$ between pion states,

$$
\left\langle\pi: \boldsymbol{P}\left|J_{\mathrm{em}}^{\mu}\right| \pi: \boldsymbol{P}^{\prime}\right\rangle=2\left(P+P^{\prime}\right)^{\mu} F\left(Q^{2}\right), \quad Q^{2} \equiv-\left(P-P^{\prime}\right)^{2} .
$$

Considering $\mu=+$ in a frame where $\boldsymbol{P}=\left(P^{+}, \mathbf{0}\right)$ and $\boldsymbol{P}^{\prime}=\left(P^{+}, \mathbf{q}_{\perp}\right)$ one is led to the the Drell-Yan formula (Drell and Yan 1970; Brodsky and Lepage 1989),

$$
F\left(\mathbf{q}_{\perp}^{2}\right)=\sum_{\lambda \lambda^{\prime}} \int_{0}^{1} d x \int \frac{d^{2} k_{\perp}}{16 \pi^{3}} \psi^{*}\left(x, \mathbf{k}_{\perp}^{\prime}, \lambda, \lambda^{\prime}\right) \psi\left(x, \mathbf{k}_{\perp}, \lambda, \lambda^{\prime}\right) .
$$

The transverse momentum of the struck quark is $\mathbf{k}_{\perp}^{\prime}=\mathbf{k}_{\perp}+(1-x) \mathbf{q}_{\perp}$. The formula (367) with its overlap of two wave functions on the right-hand-side is rather similar to the nonrelativistic result as will be shown in what follows.

The form factor of a nonrelativistic system is given by the Fourier transform of the charge distribution (normalized to one), that is,

$$
F(\mathbf{p})=\int d^{3} r \psi^{*}(\mathbf{r}) \psi(\mathbf{r}) e^{i \mathbf{p} \cdot \mathbf{x}}=\int \frac{d^{3} k}{(2 \pi)^{3}} \psi^{*}(\mathbf{k}+\mathbf{p}) \psi(\mathbf{k}) .
$$

It is important to note that $\mathbf{k}$ and $\mathbf{k}^{\prime} \equiv \mathbf{k}+\mathbf{p}$ are relative momenta,

$$
\mathbf{k} \equiv \frac{1}{M}\left(m_{2} \mathbf{k}_{1}-m_{1} \mathbf{k}_{2}\right)
$$

(and analogously for $\mathbf{k}^{\prime}$ ), so that the $\mathbf{k}_{1,2}$ are the actual particle momenta. Accordingly, $\mathbf{p}$ is the relative momentum transfer,

$$
\mathbf{p}=\mathbf{k}^{\prime}-\mathbf{k}=\frac{m_{2}}{M}\left(\mathbf{k}_{1}^{\prime}-\mathbf{k}_{1}\right) \equiv \frac{m_{2}}{M} \mathbf{q}=x_{2} \mathbf{q},
$$

where, in the last step, we have used (268). Plugging this into the form factor (368) we obtain the formula,

$$
F(\mathbf{q})=\int \frac{d^{3} k}{(2 \pi)^{3}} \psi^{*}\left(\mathbf{k}+x_{2} \mathbf{q}\right) \psi(\mathbf{k}),
$$


which, as promised, is quite similar to (367).

If one sets the momentum transfer $\mathbf{q}_{\perp}$ in (367) equal to zero, one is left with the normalization integral (352), so that the form factor is automatically normalized to one (the same is true in the nonrelativistic case).

We will use the Drell-Yan formula for the form factor to determine the pion charge radius $r_{\pi}$, which is given by the slope of the form factor at vanishing momentum transfer,

$$
F\left(q_{\perp}^{2}\right) \equiv 1-\frac{r_{\pi}^{2}}{6} q_{\perp}^{2}+O\left(q_{\perp}^{4}\right) .
$$

Using (367) this results in the nice explicit formula,

$$
r_{\pi}^{2}=-\left.\frac{3}{2} \int_{0}^{1} d x \int \frac{d^{2} k_{\perp}}{16 \pi^{3}} \frac{\partial^{2}}{\partial q_{i} \partial q_{i}} \psi_{2}^{*}\left(\mathbf{k}_{\perp}+\bar{x} \mathbf{q}_{\perp}\right)\right|_{q_{\perp}=0} \psi\left(\mathbf{k}_{\perp}\right) .
$$

Upon inserting the wave function (356), however, one encounters a problem. The sharp cutoff (corresponding to a step function) is too singular to lead to a reasonable result. The derivatives in (373) are concentrated at the boundary of the support of the wave function which in the end leads to artificial infinities. Thus, for the time being, we resort to a smooth cutoff,

$$
\theta_{\Lambda}^{s}\left(x, \mathbf{k}_{\perp}\right) \equiv \exp \left[-\frac{k_{\perp}^{2}}{\Lambda^{2} x(1-x)}\right],
$$

which basically transforms the sharp-cutoff wave function (356) to the Gaussian (286) (with $m$ set to zero). Plugging this into (373) yields the pion charge radius

$$
r_{\pi}^{2}=\frac{12}{\Lambda^{2}}=\frac{3}{4 \pi^{2} f_{\pi}^{2}}=(0.60 \mathrm{fm})^{2} .
$$

This is the standard result for the NJL model (Blin et al. 1988) and has also been obtained within the instanton model (Diakonov 1996). It is slightly smaller than the experimental value, $r_{\pi}=0.66$ (Amendolia et al. 1986), a discrepancy which is usually attributed to the use of the large-cutoff limit. A pole fit using our value of the pion charge radius is displayed in Fig. 10.

Transverse Size. As in Section 4, Example 3, we can use the light-cone wave function (356) to calculate the r.m.s. transverse momentum which leads to

$$
\left\langle k_{\perp}^{2}\right\rangle \equiv \int_{0}^{1} d x \int \frac{d^{2} k_{\perp}}{16 \pi^{3}} k_{\perp}^{2}\left\|\psi_{2 \uparrow \downarrow}\left(x, \mathbf{k}_{\perp}\right)\right\|^{2}=\frac{\Lambda^{2}}{10} \simeq(370 \mathrm{MeV})^{2} .
$$

This actually coincides with the result (288) for the smooth-cutoff Gaussian wave function (286) or (374). Therefore, unlike the charge radius $r_{\pi}$, the r.m.s. transverse momentum is insensitive to the details of the cutoff procedure. We thus have $\left\langle k_{\perp}^{2}\right\rangle^{1 / 2} \simeq m>M_{\pi}$, which confirms that the pion is highly relativistic. 
Fig. 10. The pion form factor squared vs. momentum transfer $q^{2} \equiv q_{\perp}^{2}$. The full line is the monopole fit of (Amendolia et al. 1986), $|F|^{2}=n /\left(1+q_{\perp}^{2} r_{\pi}^{2} / 6\right)^{2}$ with $n=0.991$, $r_{\pi}^{2}=0.431 \mathrm{fm}^{2}$; the dashed line is the same fit with our values, $n=1, r_{\pi}^{2}=0.36 \mathrm{fm}^{2}$. The agreement is consistent with the expected accuracy of $10 \%$.

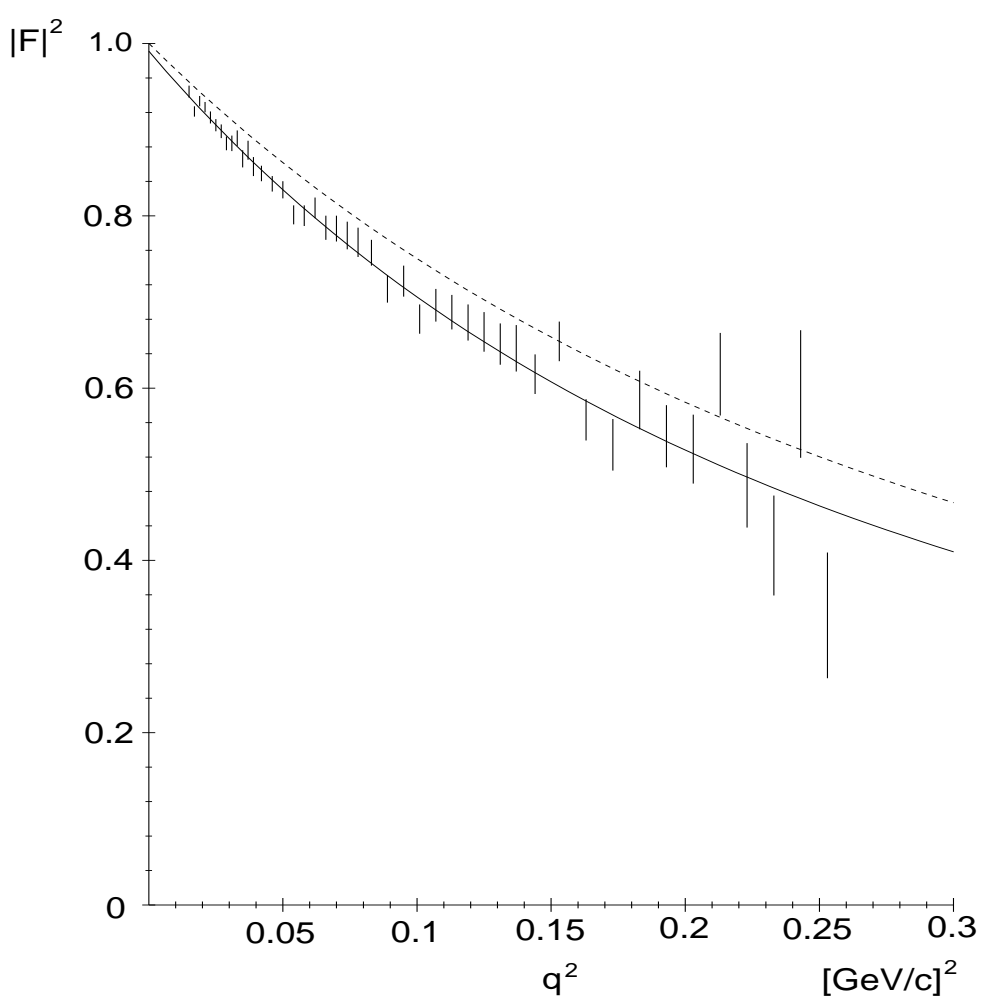

The r.m.s. transverse momentum can easily be translated into a transverse size scale $R_{\perp}$,

$$
R_{\perp}^{2} \equiv 1 /\left\langle k_{\perp}^{2}\right\rangle \simeq(0.54 \mathrm{fm})^{2}
$$

This is slightly smaller than the charge radius which we attribute to the fact that the charge distribution measured by the charge radius does not coincide with the distribution of baryon density. The 'core radius' $R_{\perp}$ is sometimes related to the decay constant $f_{\pi}$ via the dimensionless quantity $C=f_{\pi} R_{\perp}$ (Weise 1984). In constituent quark models one typically gets $C \simeq 0.4$. This implies the fairly large value $R_{\perp} \simeq 0.8 \mathrm{fm}$. Using standard many-body techniques, Bernard et al. have calculated this quantity in a model treating the pion as a collective excitation of the QCD vacuum, and find $C \simeq 0.2$ (Bernard et al. 1985). This result is close 
to what we get from $(377)$,

$$
C=f_{\pi} R_{\perp} \simeq 0.25
$$

Thus, though we work within a constituent picture, we do get a reasonable value. We believe that this is due to the intrinsic consistency of the light-cone framework with the requirements of relativity, a feature that is lacking in the constituent quark model.

(Valence) Structure Function. The pion structure function arises in the description of deep inelastic scattering off charged pions. In terms of light-cone wave functions it is defined as the momentum fraction $x$ times the sum of 'quark distributions' $f_{q}$ weighted by the quark charges $e_{q}$. The quark distributions are given by the squares of light-cone wave functions integrated over $k_{\perp}$. For the valence structure function of the pion we thus have the formula (Lepage et al. 1981; Brodsky and Lepage 1989),

$F_{2}^{v}(x)=x\left[e_{u}^{2} f_{u}^{v}(x)+e_{d}^{2} f_{\bar{d}}^{v}(x)\right]=\frac{5}{9} x f^{v}(x)=\frac{5}{9} x \sum_{\lambda \lambda^{\prime}} \int \frac{d^{2} k_{\perp}}{16 \pi^{3}}\left|\psi_{2}\left(x, \mathbf{k}_{\perp}, \lambda, \lambda^{\prime}\right)\right|^{2}$,

where the $f^{v}$ denote the valence quark distributions. For the model wave function (356) the structure function becomes

$$
F_{2}^{v}(x)=\frac{10}{3} x^{2}(1-x)
$$

which in turn leads to the (valence) quark distribution

$$
f^{v}(x) \equiv f_{u}^{v}(x)=f_{\bar{d}}^{v}(x)=6 x(1-x) .
$$

The following consistency checks can be made. The probability to find a valence quark in the pion,

$$
\int_{0}^{1} d x f^{v}(x)=1
$$

is unity, as it should. For the mean value of the momentum fraction $x$ carried by one of the quarks one finds

$$
\langle x\rangle \equiv \int_{0}^{1} d x x f^{v}(x)=1 / 2 .
$$

Thus, on average, the quarks share an equal amount of longitudinal momentum, which again, of course, is the correct result.

If one compares with other NJL calculations of the structure function (Shigetani et al. 1993; Bentz et al. 1999) and with the empirical parton distributions in the literature (Glück et al. 1999), one finds reasonable qualitative agreement. 
Let me finally point out that it is not entirely obvious to which actual momentum scale our results correspond. The transverse-momentum cutoff is $x-$ dependent, $\Lambda^{2}(x) \simeq \Lambda^{2} x(1-x)$, so, if we use the average $x$ of $(383),\langle x\rangle \simeq 1 / 2$, a natural scale seems to be ${ }^{16}$

$$
Q \equiv[\langle x\rangle(1-\langle x\rangle)]^{1 / 2} \Lambda \simeq \Lambda / 2 \simeq 600 \mathrm{MeV} .
$$

Pion Distribution Amplitude. The pion distribution amplitude was originally introduced to describe hard exclusive processes involving pions (Lepage and Brodsky 1979a; Lepage and Brodsky 1979b; Lepage and Brodsky 1980). The pion formfactor at large $Q^{2}$, for example, is given by the following convolution formula,

$$
F\left(Q^{2}\right)=\int_{0}^{1} d x \int_{0}^{1} d y \phi^{*}(x, Q) T_{H}(x, y ; Q) \phi(y, Q)[1+O(1 / Q)],
$$

where $Q$ denotes the large momentum transfer, $T_{H}$ a 'hard scattering amplitude' and $\phi$ the pion distribution amplitude. While the amplitude $T_{H}$ is the sum of all perturbative contributions to the scattering process, $\phi$ is nonperturbative in nature. The convolution formula is thus a prominent example where we see the principle of factorization into 'soft' and 'hard' physics at work.

The pion distribution amplitude is rather straightforwardly related to the light-cone wave function of the pion,

$$
\begin{aligned}
\phi(x, Q) & \sim \int \frac{d z^{-}}{4 \pi} e^{i x P^{+} z^{-} / 2}\left\langle 0\left|\bar{\psi}(0) \gamma^{+} \gamma_{5} \psi\left(z^{-}, \mathbf{0}_{\perp}\right)\right| \pi\left(P^{+}\right)\right\rangle \\
& \sim \int \frac{d^{2} k_{\perp}}{16 \pi^{3}} \psi_{q \bar{q}}^{(Q)}\left(x, \mathbf{k}_{\perp}\right) .
\end{aligned}
$$

The normalization is fixed by demanding that $\phi$ integrates to unity.

Brodsky and Lepage have shown that $\phi$ obeys an evolution equation of the form (Lepage and Brodsky 1979a; Lepage and Brodsky 1980),

$$
Q \frac{\partial}{\partial Q} \phi(x, Q)=\int_{0}^{1} d y V(x, y ; Q) \phi(y, Q) .
$$

where the evolution kernel $V$ is determined by perturbative QCD. For $Q \rightarrow \infty$, (387) has the asymptotic solution

$$
\phi_{\mathrm{as}}(x)=6 x(1-x),
$$

(which is normalized to 1). The (nonasymptotic) pion distribution amplitude has been a rather controversial object. For a while people have tended to believe in a 'double-humped' shape of the amplitude (due to a factor $(1-2 x)^{2}$ ), which was originally suggested by Chernyak and Zhitnitsky using QCD sum rules

\footnotetext{
${ }^{16}$ I thank W. Schweiger for discussions on this point.
} 
(Chernyak and Zhitnitsky 1984). In 1995, however, the CLEO collaboration has published data (Savinov 1995) that seemed to support an amplitude that is not too different from the asymptotic one (Kroll and Raulfs 1996). Theoretical evidence for this fact has recently been reported in (Belyaev and Johnson 1998a; Belyaev and Johnson 1998b; Petrov and Pobylitsa 1997; Petrov et al. 1999). Belyaev and Johnson, for instance, have found two constraints which should be satisfied by the distribution amplitude (Belyaev and Johnson 1998a),

$$
\begin{aligned}
& \phi(x=0.3)=1 \pm 0.2, \\
& \phi(x=0.5)=1.25 \pm 0.25,
\end{aligned}
$$

which are consistent with an amplitude being close-to-asymptotic.

Last year, the experimental developments have culminated in a direct measurement of the distribution amplitude at Fermilab (Ashery 1999). At a fairly low (i.e. nonasymptotic) momentum scale of $Q^{2} \simeq 10 \mathrm{GeV}^{2}$, one finds a distribution amplitude that is very close to the asymptotic one.

Fig. 11. The (asymptotic) pion distribution amplitude. The vertical lines mark the constraints (389) of Belyaev and Johnson.

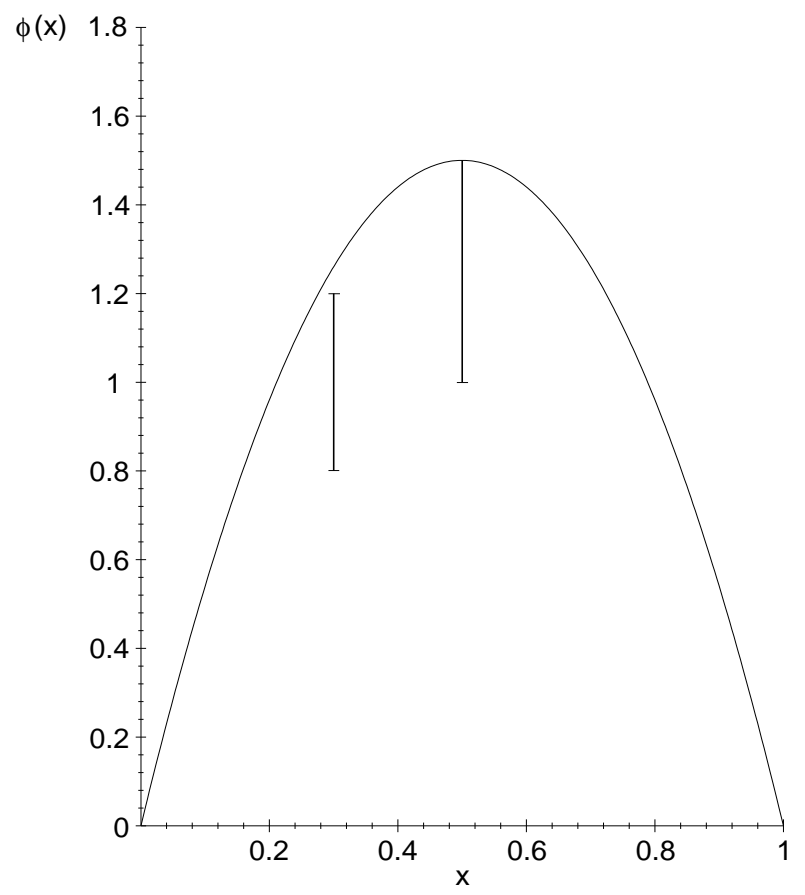


Let us see what we get in the NJL model. The distribution amplitude is given by

$$
\phi_{\mathrm{NJL}}(x)=\frac{2 \sqrt{3}}{f_{\pi}} \int \frac{d^{2} k_{\perp}}{16 \pi^{3}} \psi_{2 \uparrow \downarrow}\left(x, \mathbf{k}_{\perp}\right)=6 x(1-x)=\phi_{\mathrm{as}}(x) .
$$

Thus, in the large cutoff limit, the NJL distribution amplitude is exactly given by the asymptotic one! Upon comparing with (381), we see that $\phi_{\mathrm{NJL}}$ coincides with the quark distribution $f^{v}$. This is accidental and stems from the fact that, due to the use of a step function in (356), $\psi_{2} \sim\left|\psi_{2}\right|^{2}$.

In Figure 11 we have displayed our distribution amplitude together with the constraints (389), represented by the vertical lines.

Our findings are thus consistent with the recent Fermilab experiment (Ashery 1999). One should, however, be aware of the fact that our energy scale of $Q \simeq 0.6$ $\mathrm{GeV}$ from (384) is below the experimental one of $Q \simeq 3 \mathrm{GeV}$. It is also somewhat lower than $Q \simeq 1 \mathrm{GeV}$, which has been assumed by Belyaev and Johnson in their analysis of the distribution amplitude in terms of light-cone quark models (Belyaev and Johnson 1998b).

\section{Conclusions}

In this lecture I have discussed an alternative approach to relativistic (quantum) physics based on Dirac's front form of dynamics. It makes use of the fact that for relativistic systems the choice of the time parameter is not unique. Our particular choice uses null-planes tangent to the light-cone as hypersurfaces of equal time. This apparently trivial change of coordinates has far-reaching consequences:

- The number of kinematical (i.e. interaction independent) Poincaré generators becomes maximal; there are seven of them instead of the usual six, among them the boosts.

- Lorentz boosts in $z$-direction become diagonal; the light-cone time and space coordinates, $x^{+}$and $x^{-}$, respectively, do not get mixed but rather get rescaled.

- As a consequence, for many-particle systems one can introduce frame-independent relative coordinates, the longitudinal momentum fractions, $x_{i}$, and the relative transverse momenta, $\mathbf{k}_{\perp i}$.

- Because of a two-dimensional Galilei invariance, relative and center-of-mass motion separate. As a result, many formulae are reminiscent of nonrelativistic physics and thus very intuitive.

- This is particularly true for light-cone wave functions which, due to the last two properties, are boost invariant and do not depend on the total momentum of the bound state. They are therefore ideal tools to study relativistic particle systems.

- The last statement even holds for relativistic quantum field theory where one combines the unique properties of light-cone quantization with a Fock space picture. The central feature making this a reasonable idea is the triviality 
of the light-cone vacuum which accordingly is an eigenstate of the fully interacting Hamiltonian. This implies that the Fock operators create the physical particles from the ground state.

As expected, however, the principle of conservation of difficulties is at work so that there are problems to overcome. Of particular concern to us was the 'vacuum problem'. In instant-form quantum field theory, especially in QCD, many nonperturbative phenomena are attributed to the nontriviality of the vacuum which shows up via the appearance of condensates. These suggest that the instant-form vacuum is a complicated many-body state (like, e.g. the BCS ground state). In addition, many of these condensates signal the spontaneous (or anomalous) breakdown of a symmetry. The conceptual problem which arises at this point is to reconcile the existence of condensates with the triviality of the light-cone vacuum.

The idea put forward in these lectures is to reconstruct ground state properties from the particle spectrum. The latter is obtained by solving the light-cone Schrödinger equation for masses and wave functions of the associated bound states. For a relativistic quantum field theory, this, in principle, amounts to solving an infinite system of coupled integral equations for the amplitudes to find an ever increasing number of constituents in the bound state. Experience, however, shows that the light-cone amplitudes to find more than the valence quanta in the bound state tend to become rather small. Note that the same is not true within ordinary, that is, instant-form quantization.

A first explorative step towards solving a realistic light-cone Schrödinger equation was performed using an effective field theory, the NJL model. We have seen that, though we made a number of approximations, in particular by enforcing a constituent picture, a number of pionic observables are predicted with reasonable accuracy, among them the pion electromagnetic form factor, the pion charge and core radius and the pion valence structure function at low normalization scale. The pion distribution amplitude (in the chiral and large-cutoff limit) turns out to be asymptotic.

The results presented in these lectures provide some confidence that, also for real QCD, light-cone quantization may provide a road towards a reasonable constituent picture, in which hadrons are consistently described as bound states of a minimal number of constituents. How this hope can be turned into fact remains to be seen.

\section{Acknowledgments}

It is a pleasure to thank the organizers of the 39th Schladming winter school, in particular W. Schweiger and H. Latal, for inviting me to lecture and providing such a stimulating environment, both scientifically and socially. 


\section{References}

Abdalla, E. and M. C. B. Abdalla (1996). Updating QCD in two dimensions. Phys. Rept. 265, 253.

Amendolia, S. R. et al. (1986). A measurement of the space-like pion electromagnetic form factor. Nucl. Phys. B277, 168.

Antonuccio, F., S. J. Brodsky, and S. Dalley (1997). Light cone wave functions at small x. Phys. Lett. B412, 104.

Ashery, D. (1999). Diffractive dissociation of high momentum pions. hep-ex/9910024.

Banks, T. (1999) TASI lectures on matrix theory. hep-th/9911068.

Banks, T., W. Fischler, S. Shenker, and L. Susskind (1997). M theory as a matrix model: A conjecture. Phys. Rev. D 55, 5112.

Bardakci, K., M.B. Halpern (1968). Theories at infinite momentum. Phys. Rev. 176, 1686.

Bardeen, W. A., R. B. Pearson, and E. Rabinovici (1980). Hadron masses in Quantum Chromodynamics on the transverse lattice. Phys. Rev. D21, 1037.

Bassetto, A., G. Nardelli, and R. Soldati (1991). Yang-Mills theories in algebraic noncovariant gauges: Canonical quantization and renormalization. World Scientific, Singapore.

Bassetto, A., F. Vian, and L. Griguolo (2000). Light-front vacuum and instantons in two dimensions. hep-th/0004026.

Belyaev, V. and M. Johnson (1998a). Twist-2 light-cone pion wave function. Mod. Phys. Lett. A13, 2909.

Belyaev, V. and M. Johnson (1998b). Pion light-cone wave functions and light-front quark model. Phys. Lett. B423, 379.

Bentz, W., T. Hama, T. Matsuki, and K. Yazaki (1999). NJL model on the light-cone and pion structure function. Nucl. Phys. A651, 143.

Bernard, V., R. Brockmann, and W. Weise (1985). The Goldstone pion and the quarkantiquark pion (II). Pion size and decay. Regensburg Preprint, TPR 85-02.

Bethe, H. and E. Salpeter (1957). Quantum Mechanics of One- and Two-Electron Atoms. Springer, Berlin, Heidelberg, New York.

Bjorken, J. and S. Drell (1964). Relativistic Quantum Mechanics. McGraw-Hill.

Blin, A. H., B. Hiller, and M. Schaden (1988). Electromagnetic form factors in the Nambu-Jona-Lasinio model. Z. Phys. A331, 75.

Bogolubov, N., A. Logunov, A. Oksak, and I. Todorov (1990). General Principles of Quantum Field Theory. Kluwer Academic.

Bogolubov, N., A. Logunov, and I. Todorov (1975). Introduction to Axiomatic Field Theory. Benjamin, New York.

Borderies, A., P. Grange, and E. Werner (1993). Light-cone quantization and 1/N expansion. Phys. Lett. B319, 490.

Borderies, A., P. Grange, and E. Werner (1995). Broken phase of the $O(N) \phi^{4}$ model in light-cone quantization and $1 / N$ expansion. Phys. Lett. B345, 458.

Brodsky, S., C.-R. Ji, and M. Sawicki (1985). Evolution equation and relativistic bound-state wave functions for scalar field models in four and six dimensions. Phys. Rev. D32, 1530.

Brodsky, S. and G. Lepage (1989). Exclusive processes in Quantum Chromodynamics. in: Perturbative Quantum Chromodynamics, A.H. Mueller, ed., World Scientific, Singapore. 
Brodsky, S. J., H.-C. Pauli, and S. S. Pinsky (1998). Quantum Chromodynamics and other field theories on the light-cone. Phys. Rept. 301, 299.

Brodsky, S. J., R. Roskies, and R. Suaya (1973). Quantum electrodynamics and renormalization theory in the infinite momentum frame. Phys. Rev. D8, 4574.

Burkardt, M. (1996). Parity invariance and effective light-front hamiltonians. Phys. Rev. D54, 2913.

Burkardt, M. (1998). Finiteness conditions for light front-hamiltonians. Phys. Rev. D57, 1136.

Callan, C., N. Coote, and D. Gross (1976). Two-dimensional Yang-Mills theory: A model of quark confinement. Phys. Rev. D13, 1649.

Casher, A. (1976). Gauge fields on the null plane. Phys. Rev. D14, 452.

Casher, A., S. Noskowicz, and L. Susskind (1971). Goldstone-Nambu bosons in dual and parton theories. Nucl. Phys. B32, 75.

Casher, A. and L. Susskind (1974). A quark model of mesons based on chiral symmetry. Phys. Rev. D9, 436.

Chakrabarti, D., A. Mukherjee, R. Kundu, and A. Harindranath (1999). A numerical experiment in DLCQ: Microcausality, continuum limit and all that. Phys.Lett. B480, 409.

Chakrabarty, S., K. Gupta, N.N. Singh, and A.N. Mitra (1989). Hadron spectroscopy and form factors at quark level. Prog. Part. Nucl. Phys. 22, 43.

Chang, S.-J., R. G. Root, and T.-M. Yan (1973). Quantum field theories in the infinite momentum frame. I. Quantization of scalar and Dirac fields. Phys. Rev. D7, 1133.

Chernyak, V. and A. Zhitnitsky (1984). Asymptotic behaviour of exclusive processes in QCD. Phys. Rept. 112, 173.

Chung, P., F. Coester, and W. Polyzou (1988). Charge form factors of quark-model pions. Phys. Lett. B205, 545.

Coester, F. (1992). Null-plane dynamics of particles and fields. Prog. Part. Nucl. Phys. 29, 1.

Coester, F. and W. Polyzou (1982). Relativistic quantum mechanics of particles with direct interaction. Phys. Rev. D26, 1348.

Coleman, S. (1973). There are no Goldstone bosons in two dimensions. Comm. Math. Phys. 31, 259.

Courant, R. and D. Hilbert (1962). Methods of Mathematical Physics. Interscience, New York.

Dancoff, S. (1950). Nonadiabatic meson theory of nuclear forces. Phys. Rev. 78, 382.

DeWitt, B. (1984). The space-time approach to quantum field theory. in: Relativity, Groups and Topology, II, B.S. DeWitt and R. Stora, eds., Proceedings Les Houches 1983, Session XL, North-Holland, Amsterdam.

Diakonov, D. (1996). Chiral symmetry breaking by instantons. in: Selected Topics in Nonperturbative $Q C D$, A. Di Giacomo and D. Diakonov, eds., Proceedings International School of Physics "Enrico Fermi", Course CXXX, Varenna, Italy, 1995, IOS Press, Amsterdam.

Dietmaier, C., T. Heinzl, M. Schaden, and E. Werner (1989). The fermion condensate of the Nambu-Jona-Lasinio model in light-cone quantization. Z. Phys. A334, 220.

Dirac, P. A. M. (1949). Forms of relativistic dynamics. Rev. Mod. Phys. 21, 392.

Dirac, P. A. M. (1964). Lectures on quantum mechanics. Benjamin, New York.

Domokos, G. (1972). Introduction to the characteristic initial-value problem in quantum field theory. in: Boulder Lectures, Vol. XIV, A.O. Barut, W.E. Brittin, eds., Colorado University Press, Boulder. 
Dosch, H. and S. Narison (1998). Direct extraction of the chiral quark condensate and bounds on the light quark masses. Phys. Lett. B417, 173.

Drell, S. and T.-M. Yan (1970). Connection of elastic electromagnetic nucleon form factors at large $q^{2}$ and deep inelastic structure functions near threshold. Phys. Rev. Lett. 24, 181.

Dziembowski, Z. (1988). Relativistic model of nucleon and pion structure: Static properties and electromagnetic soft form factors. Phys. Rev. D37, 778.

Faddeev, L. and R. Jackiw (1988). Hamiltonian reduction of unconstrained and constrained systems. Phys. Rev. Lett. 60, 1692.

Fock, V. (1935). Zur Theorie des Wasserstoffatoms. Z. Phys. 98, 145.

Fubini, S., A. Hanson, and R. Jackiw (1973). New approach to field theory. Phys. Rev. D\%, 1732.

Fuda, M. (1990). A new picture for light-front dynamics. Ann. Phys. (N.Y.) 197, 265.

Gelfand, I. and G. Shilov (1964). Generalized Functions. Academic Press, New York.

Gell-Mann, M., R. J. Oakes, and B. Renner (1968). Behavior of current divergences under $S U(3) \times S U(3)$. Phys. Rev. 175, 2195.

Gitman, D. and I. Tyutin (1990). Quantization of Fields with Constraints. Springer, Berlin, Heidelberg, New York.

Glück, M., E. Reya, and I. Schienbein (1999). Pionic parton distributions revisited. Eur. Phys. J. C10, 313.

Goldstone, J. (1961). Field theories with 'superconductor' solutions. Nuovo Cim. 19, 154.

Green, M., J. Schwarz, and E. Witten (1987). Superstring Theory. Cambridge University Press, Cambridge.

Gribov, V. N. (1978). Quantization of non-Abelian gauge theories. Nucl. Phys. B139, 1.

Gross, F. (1989) Relativistic nuclear physics with the spectator model. in: Nuclear and Particle Physics on the Light-Cone, Proceedings, LAMPF Workshop, Los Alamos, 1988, M.B. Johnson, L.S. Kisslinger, eds., World Scientific, Singapore.

Hanson, A., T. Regge, and C. Teitelboim (1976). Constrained Hamiltonian Systems. Accademia Nazionale dei Lincei, Rome.

Harada, K., T. Heinzl, and C. Stern (1998). Variational mass perturbation theory for light-front bound-state equations. Phys. Rev. D57, 2460.

Harada, K., T. Sugihara, M. Taniguchi, and M. Yahiro (1994). The massive Schwinger model with $S U(2)_{f}$ on the light-cone. Phys. Rev. D49, 4226.

Harindranath, A., R.J. Perry, and K.G. Wilson (1990). Light-front Tamm-Dancoff field theory. Phys. Rev. Lett. 65, 2959.

Hatsuda, T. and T. Kunihiro (1994). QCD phenomenology based on a chiral effective Lagrangian. Phys. Rept. 247, 221.

Heinzl, T. (1996a). Hamiltonian formulations of Yang-Mills quantum theory and the Gribov problem. hep-th/9604018.

Heinzl, T. (1996b). Fermion condensates and the trivial vacuum of light-cone quantum field theory. Phys. Lett. B388, 129.

Heinzl, T. (1998). Light-cone dynamics of particles and fields. hep-th/9812190.

Heinzl, T., S. Krusche, S. Simbürger, and E. Werner (1992). Nonperturbative lightcone quantum field theory beyond the tree level. Z. Phys. C56, 415.

Heinzl, T., N. Scheu, and H. Kröger (1999). Loss of causality in discretized light-cone quantization. hep-th/9908173. 
Heinzl, T., C. Stern, E. Werner, and B. Zellermann (1996). The vacuum structure of light-front $\phi_{1+1}^{4}$ theory. Z. Phys. Cr2, 353.

Heinzl, T. and E. Werner (1994). Light-front quantization as an initial-boundaryvalue problem. Z. Phys. C62, 521.

Hellerman, S. and J. Polchinski (1999). Compactification in the lightlike limit. Phys. Rev. D 59, 125002.

Hiller, J. (2000). Calculations with DLCQ. to appear in the Proceedings of 10th International Light Cone Meeting on Non-Perturbative QCD and Hadron Phenomenology: From Hadrons to Strings, Heidelberg, Germany, June 12-16, 2000, hep-ph/0007309.

Holstein, B. R. (1995). Chiral perturbation theory: A primer. hep-ph/9510344.

Holstein, B. R. (1998). Klein's paradox. Am. J. Phys. 66, 507.

Hornbostel, K. (1992). Nontrivial vacua from equal time to the light-cone. Phys. Rev. D45, 3781 .

Hornbostel, K., S. J. Brodsky, and H.-C. Pauli (1988). Quantization on the light-cone: Response to a comment by Hagen. Phys. Rev. D37, 2363.

Itakura, K. and S. Maedan (2000). Dynamical chiral symmetry breaking on the lightfront. I: DLCQ approach. Phys. Rev. D61, 045009.

Jackiw, R. (1987). Functional representations for quantized fields. in: Conformal Field Theory, Anomalies, and Superstrings, C.K. Chew et al., eds., Proceedings of the First Asia Pacific Workshop on High Energy Physics, Singapore, 1987, World Scientific, Singapore.

Jackiw, R. (1993). (Constrained) quantisation without tears. hep-th/9306075.

Jaen, X., A. Molina, and J. Llosa (1984). Front form predictive mechanics noninteraction theorem. in: Lecture Notes in Physics, 212, Springer, Berlin, Heidelberg, New York.

Jaus, W. (1990). Semileptonic decays of B and D mesons in the light-front formalism. Phys. Rev. D41, 3394.

Ji, C.-R., P. Chung, and S. Cotanch (1992). Light-cone quark-model axial-vector wave function. Phys. Rev. D45, 4214.

Ji, C.-R. and S. Cotanch (1990). Simple relativistic quark-model analysis of flavored pseudoscalar mesons. Phys. Rev. D41, 2319.

Jordan, P. and W. Pauli (1928). Zur Quantenelektrodynamik ladungsfreier Felder. Z. Phys. 47, 151.

Kaplan, D. (1995). Effective field theories. nucl-th/9506035.

Kiefer, C. and A. Wipf (1994). Functional Schrödinger equation for fermions in external gauge fields. Ann. Phys. (N.Y.) 236, 241.

Klevansky, S. P. (1992). The Nambu-Jona-Lasinio model of Quantum Chromodynamics. Rev. Mod. Phys. 64, 649.

Kroll, P. and M. Raulfs (1996). The $\pi \gamma$ transition form factor and the pion wave function. Phys. Lett. B387, 848.

Lavelle, M., E. Werner, and S. Glazek (1987). Hadron wave functions with condensate induced running masses. Few Body Systems, Suppl. 2, 519.

Leibbrandt, G. (1984). The light-cone gauge in Yang-Mills theory. Phys. Rev. D29, 1699.

Lenz, F., M. Thies, K. Yazaki, and S. Levit (1991). Hamiltonian formulation of twodimensional gauge theories on the light-cone. Ann. Phys. (N.Y.) 208, 1.

Lepage, G. and S. Brodsky (1980). Exclusive processes in Quantum Chromodynamics. Phys. Rev. D22, 2157. 
Lepage, G., S. Brodsky, T. Huang, and P. Mackenzie (1981). Hadronic wave functions in QCD. Proceedings of the Banff Summer Institute.

Lepage, G. P. and S. J. Brodsky (1979a). Exclusive processes in Quantum Chromodynamics: Evolution equations for hadronic wave functions and the form factors of mesons. Phys. Lett. B87, 359.

Lepage, G. P. and S. J. Brodsky (1979b). Exclusive processes in Quantum Chromodynamics: the form factors of baryons at large momentum transfer. Phys. Rev. Lett. 43, 545.

Leutwyler, H. (1965). A no-interaction theorem in classical relativistic Hamiltonian particle mechanics. Nuovo Cim. 37, 556.

Leutwyler, H. (1974a). On the average transverse momentum of the quarks within a meson. Phys. Lett. B48, 45.

Leutwyler, H. (1974b). Mesons in terms of quarks on a null-plane. Nucl. Phys. B76, 413.

Leutwyler, H. and J. Stern (1978). Relativistic dynamics on a null-plane. Ann. Phys. (N.Y.) 112, 94.

Liu, H. H. and D. E. Soper (1993). Implementation of the Leibbrandt-Mandelstam gauge prescription in the null-plane bound-state equation. Phys. Rev. D48, 1841.

Lucha, W., F. Schöberl, and D. Gromes (1991). Bound states of quarks. Phys. Rept. 200, 127.

Lunin, O. and S..S. Pinsky (1999). SDLCQ: Supersymmetric discrete light-cone quantization. in: New Directions in Quantum Chromodynamics, C.-R. Ji and D.-P. Min, eds., AIP Conference Proceedings 494, Melville, NY.

Mandelstam, S. (1983). Light-cone superspace and the ultraviolet finiteness of the $N=4$ model. Nucl. Phys. B213, 149.

Manohar, A. (1997). Effective field theories. in: Perturbative and Nonperturbative Aspects of Quantum Field Theory, H. Latal and W. Schweiger, eds., Lecture Notes in Physics, Vol. 479, p. 311, Springer, Berlin, Heidelberg, New York.

Manohar, A. and H. Georgi (1984). Chiral quarks and the nonrelativistic quark model. Nucl. Phys. B234, 189.

Maris, P. and P.C. Tandy (2000). The quark photon vertex and the pion charge radius. Phys.Rev. C61, 045202.

Maskawa, T. and K. Yamawaki (1976). The problem of $p^{+}=0$ mode in the null-plane field theory and Dirac's method of quantization. Prog. Theor. Phys. 56, 270.

McCartor, G. and D.G. Robertson (1992). Bosonic zero modes in discretized light-cone field theory. Z. Phys. C53, 679.

Melosh, H. (1974). Quarks: Currents and constituents. Phys.Rev. D9, 1095.

Michael, C. and F. Payne (1982). Bound states of heavy quarks on the light plane. Z. Phys. C12, 145.

Mukherjee, S., R. Nag, S. Sanyal, T. Morii, J. Morishita and M. Tsuge (1993). Quark potential approach to baryons and mesons. Phys. Rept. 231, 201.

Nakanishi, N. and K. Yamawaki (1977). A consistent formulation of the null-plane quantum field theory. Nucl. Phys. B122, 15.

Nambu, Y. and G. Jona-Lasinio (1961). Dynamical model of elementary particles based on an analogy with superconductivity. I. Phys. Rev. 122, 345.

Namysłowski, J. (1985). Light-cone perturbation theory and its application to different fields. Prog. Part. Nucl. Phys. 14, 49.

Neubert, M. (1994). Heavy quark symmetry. Phys. Rept. 245, 259. 
Neville, R. and F. Rohrlich (1971). Quantum field theory off null-planes. Nuovo Cim. 1A, 625.

Newton, T. and E. Wigner (1949). Localized states for elementary systems. Rev. Mod. Phys. 21, 400.

Parker, L. and G. Schmieg (1970). Special relativity and diagonal transformations. Am. J. Phys. 38, 218.

Pauli, H.-C. and S. J. Brodsky (1985a). Solving field theory in one space and one time dimension. Phys. Rev. D32, 1993.

Pauli, H.-C. and S. J. Brodsky (1985b). Discretized light-cone quantization: Solution to a field theory in one space and one time dimension. Phys. Rev. D32, 2001.

Peierls, R. (1952). The commutation laws of relativistic field theory. Proc. Roy. Soc. A214, 143.

Perry, R. J. (1999). Light-front Quantum Chromodynamics. nucl-th/9901080.

Petrov, V. Y. and P. Pobylitsa (1997). Pion wave function from the instanton vacuum model. hep-ph/9712203.

Petrov, V. Y., M. V. Polyakov, R. Ruskov, C. Weiss, and K. Goeke (1999). Pion and photon light-cone wave functions from the instanton vacuum. Phys. Rev. D59, 114018.

Polyakov, A. (1987). Gauge Fields and Strings. Harwood Academic, Chur.

Prokhvatilov, E. and V. Franke (1988). Approximate description of QCD condensates in light-cone coordinates. Sov. J, Nucl. Phys. 47, 559.

Prokhvatilov, E. and V. Franke (1989). Limiting transition to light-like coordinates and the QCD Hamiltonian. Sov. J. Nucl. Phys. 49, 688.

Radyushkin, A.V. (1995). Quark-hadron duality and intrinsic transverse momentum. Acta Phys. Polon. B26, 2067.

Roberts, C. D. and A. G. Williams (1994). Dyson-Schwinger equations and their application to hadronic physics. Prog. Part. Nucl. Phys. 33, 477.

Roberts, C. D. and S. M. Schmidt (2000). Dyson-Schwinger equations: density, temperature and continuum strong QCD. nucl-th/0005064.

Rohrlich, F. (1971). Null-plane field theory. Acta Physica Austriaca, Suppl. 8, 277.

Salmons, S., P. Grange, and E. Werner (1999). Field dynamics on the light-cone: Compact versus continuum quantization. Phys. Rev. D 60, 067701.

Savinov, V. (1995). A measurement of the form factors of light pseudoscalar mesons at a large momentum transfer. hep-ex/9507005.

Şavklı, Ç. and F. Tabakin (1998). Quark-antiquark bound states within a DysonSchwinger Bethe-Salpeter formalism. Nucl. Phys. A 628, 645.

Scheu, N. (1997). On the computation of structure functions and mass spectra in a relativistic Hamiltonian formalism: A lattice point of view. Ph.D. Thesis, Université Laval, Québec. hep-th/9804190.

Schwinger, J. (1949). Quantum Electrodynamics. II. Vacuum polarization and selfenergy. Phys. Rev. 75, 651.

Schwinger, J. (1951). The theory of quantized fields. I. Phys. Rev. 82, 914.

Schwinger, J. (1953a). The theory of quantized fields. II. Phys. Rev. 91, 713.

Schwinger, J. (1953b). A note on the quantum dynamical principle. Phil. Mag. 44, 1171.

Shifman, M. A., A. I. Vainshtein, and V. I. Zakharov (1979). QCD and resonance physics. Sum rules. Nucl. Phys. B147, 385.

Shigetani, T., K. Suzuki, and H. Toki (1993). Pion structure function in the Nambu and Jona-Lasinio model. Phys. Lett. B308, 383. 
Sokolov, S. and A. Shatnii (1979). Physical equivalence of the three forms of relativistic dynamics and addition of interactions in the front and instant form. Theor. Math. Phys. 37, 1029.

Srivastava, P. P. and S. J. Brodsky (2000). Light-front-quantized QCD in covariant gauge. Phys. Rev. D61, 025013.

C. Stern (1999) Chirale Symmetriebrechung und gebundene Zustände in Lichtkegelquantenfeldtheorien. PhD thesis, Regensburg, (unpublished).

Streater, R. and A. Wightman (1963). PCT, Spin and Statistics, and all that. Benjamin, New York.

Sundermeyer, K. (1982). Constrained Dynamics. Springer, Berlin, Heidelberg, New York.

Susskind, L. (1968). Model of self-induced strong interactions. Phys. Rev. 165, 1535.

Susskind, L. (1997). Another conjecture about M(atrix) theory. hep-th/9704080.

't Hooft, G. (1974). A two-dimensional model for mesons. Nucl. Phys. B75, 461.

't Hooft, G. (1975). Gauge theory for strong interactions. Lecture given at the International School of Subnuclear Physics, Erice, Italy, 1975; in: New Phenomena in Subnuclear Physics, Part A, A. Zichichi, ed., Plenum, New York.

Tamm, I. (1945). Relativistic interaction of elementary particles. J. Phys. (Moscow) 9, 449.

Terent'ev, M. (1976). On the structure of the wave functions of mesons considered as bound states of relativistic quarks. Sov. J. Nucl. Phys. 24, 106.

Tomboulis, E. (1973). Quantization of the Yang-Mills field in the null-plane frame. Phys. Rev. D8, 2736.

Vogl, U. and W. Weise (1991). The Nambu and Jona-Lasinio model: Its implications for hadrons and nuclei. Prog. Part. Nucl. Phys. 27, 195.

Weise, W. (1984). Quarks, chiral symmetry and dynamics of nuclear constituents. in: International Review of Nuclear Physics, Vol.1, Quarks and Nuclei, W. Weise, ed., World Scientific, Singapore.

Wilson, K.G., T.S. Walhout, A. Harindranath, W.-M. Zhang, R.J. Perry, and S.D. Glazek (1994). Nonperturbative QCD: A weak coupling treatment on the lightfront. Phys. Rev. D49, 6720.

Witten, E. (1978). Chiral Symmetry, the $1 / N$ expansion and the $S U(N)$ Thirring model. Nucl. Phys. B145, 110.

Wittman, R.S. (1989) Symmetry breaking in the $\left(\phi^{4}\right)_{2}$ theory and the light-front vacuum. in: Nuclear and Particle Physics on the Light-Cone, Proceedings, LAMPF Workshop, Los Alamos, 1988, M.B. Johnson, L.S. Kisslinger, eds., World Scientific, Singapore.

Wu, T.T. (1977). Two-dimensional Yang-Mills theory in the leading $1 / N$ expansion. Phys. Lett. 71B, 142.

Yndurain, F. (1996). Relativistic Quantum Mechanics and Introduction to Field Theory. Springer, Berlin, Heidelberg, New York.

Zhitnitsky, A. (1986). On chiral symmetry breaking in $\mathrm{QCD}_{2}\left(N_{C} \rightarrow \infty\right)$. Sov. J. Nucl. Phys. 43, 999.

Zinn-Justin, J. (1996). Quantum Field Theory and Critical Phenomena. Clarendon Press, Oxford. 